Aus der Abteilung für Orthopädie

(Prof. Dr. med. W. Schultz)

im Zentrum Chirurgie

der Medizinischen Fakultät der Universität Göttingen

\title{
Das Problem der Ossifikation und Implantat- Wanderung nach VEPTR (vertical expandable prosthetic titanium rib)-Implantation bei Kindern und Jugendlichen mit Thorax-Insuffizienz-Syndrom und Skoliose
}

\author{
INAUGURAL-DISSERTATION \\ zur Erlangung des Doktorgrades \\ der Medizinischen Fakultät \\ der Georg-August-Universität zu Göttingen
}

vorgelegt von

BASTIAN GRÖNEFELD

aus Nordhorn

Göttingen 2012 
Dekan:

1. Berichterstatterin:

2. Berichterstatter:

Tag der mündlichen Prüfung:
Prof. Dr. med. M. P. Schön

Prof. Dr. med. A.-K. Hell

Prof. Dr. med. H.-C. Ludwig

13.08 .2012 


\section{Inhaltsverzeichnis}

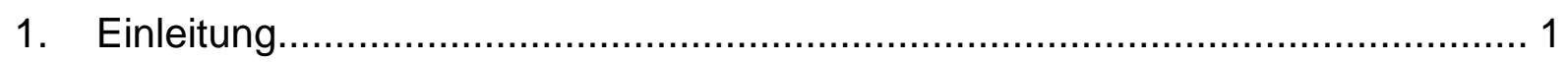

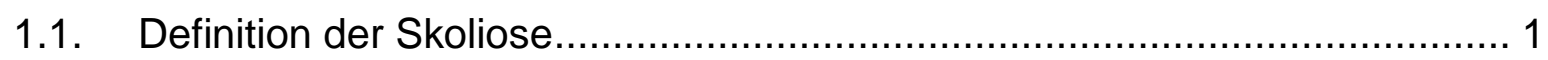

1.2. Ätiologie und Epidemiologie der Skoliose ............................................. 1

1.2.1. Idiopathische Skoliose ............................................................. 1

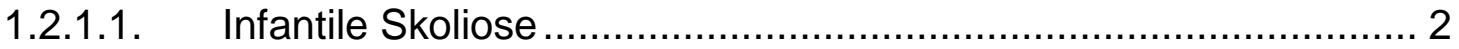

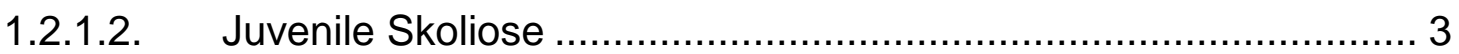

1.2.1.3. Adoleszente Skoliose ............................................................ 3

1.2.2. Neurogene Skoliose ................................................................ 4

1.2.2.1. Spinale Muskelatrophie und Skoliose ...................................... 4

1.2.2.2. Myelomeningozele und Skoliose ............................................ 5

1.2.3. Kongenitale Skoliose ................................................................. 7

1.3. Das thoracic insufficiency syndrome ………......................................... 7

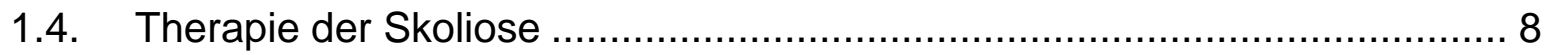

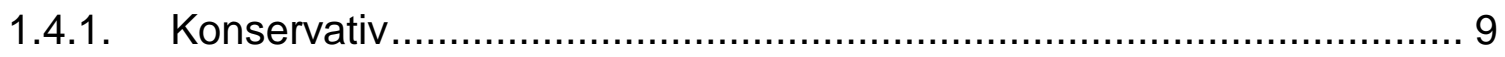

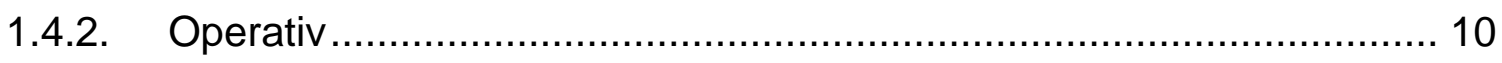

1.4.2.1. Dorsale Spondylodese ........................................................ 10

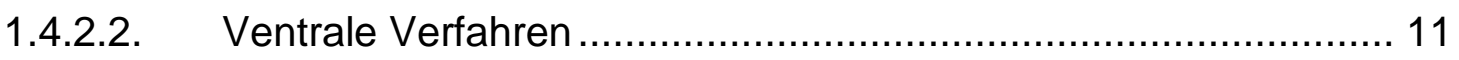

1.4.2.3. Traditionelle Verfahren bei Kindern < 10 Jahre .......................... 12

1.4.2.4. Expandierende „mitwachsende“ Systeme................................. 13

1.5. Das VEPTR (vertical expandable prosthetic titanium rib)-Implantat........... 14

1.5.1. Geschichte und Entwicklung ….................................................... 14

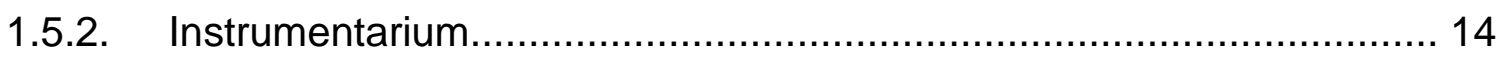

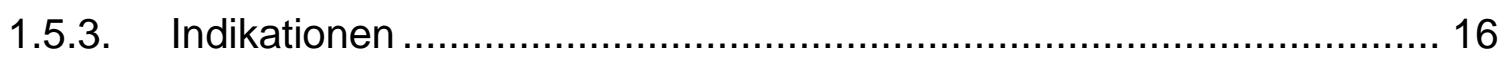

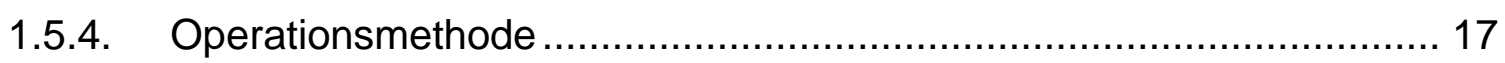

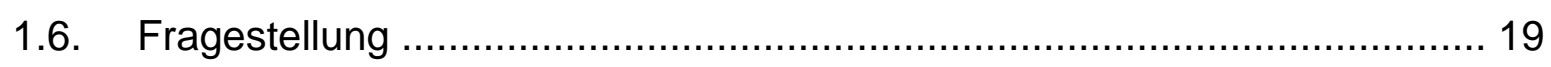

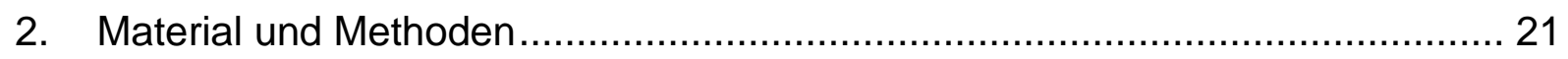

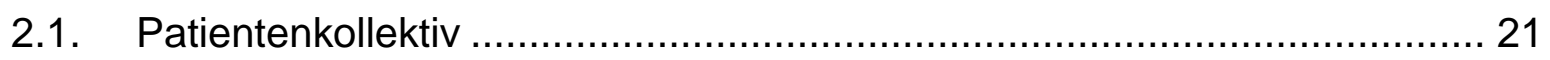

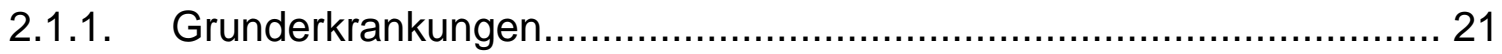

2.1.2. Operative Versorgung ................................................................ 21 
2.1.3. Operativer Verlauf während der Nachuntersuchung......................... 23

2.2. Untersuchung der Wirbelsäulenaufnahmen ......................................... 24

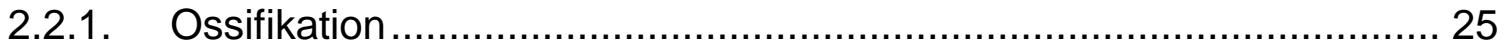

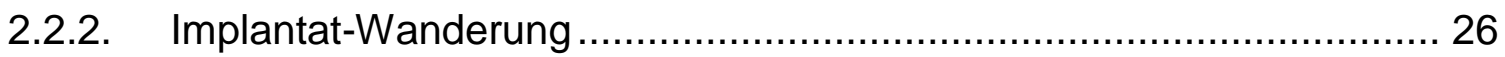

2.2.3. Sonstige Komplikationen/radiologische Parameter ........................... 27

2.2.4. Einflussgrößen auf die radiologischen Parameter ............................. 28

2.3. Statistische Auswertung der Komplikationen/radiologischen Parameter.... 29

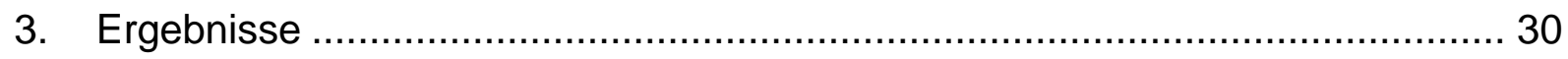

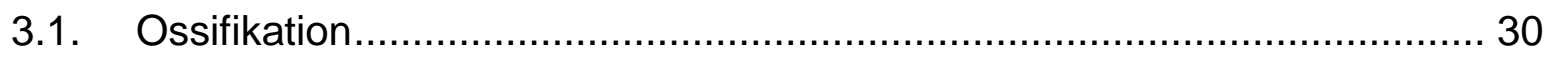

3.1.1. Inzidenz und Lokalisation ........................................................ 30

3.1.2. Zeitpunkt und Wahrscheinlichkeit............................................... 31

3.1.3. Größe der Ossifikationen im Verlauf der Behandlung ........................ 33

3.1.4. Einflussgrößen auf die Ausbildung von Ossifikationen ....................... 34

3.1.5. Vergleich der Behandlungsdauer, der Operationsanzahl und der Komplikationen ........................................................................ 36

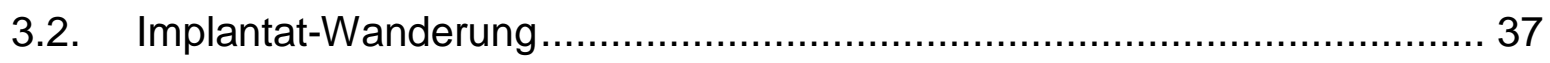

3.2.1. Inzidenz und Lokalisation ............................................................. 37

3.2.2. Strecke im Verlauf der Behandlung ............................................. 37

3.2.3. Einflussgrößen auf das Entstehen der Implantat-Wanderung ............ 42

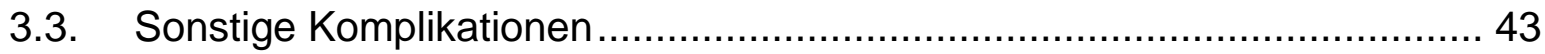

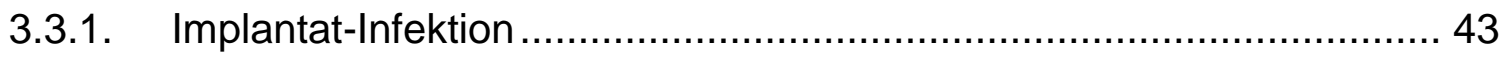

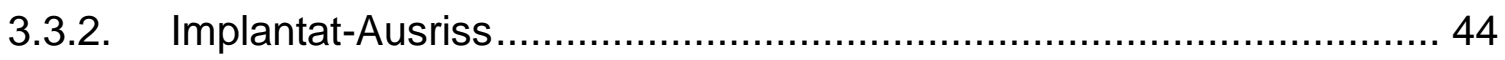

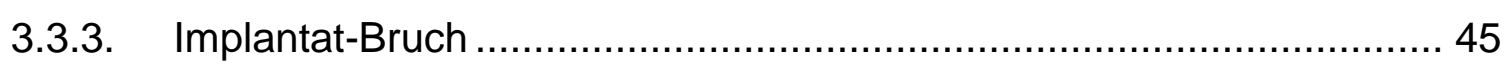

3.3.4. Rippen-Spreizung nach Primär-Implantation................................... 46

3.4. Operativer Verlauf während der Nachuntersuchung …........................... 47

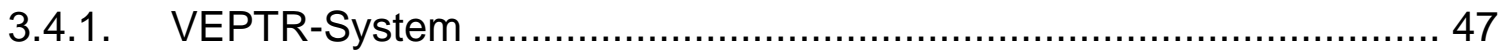

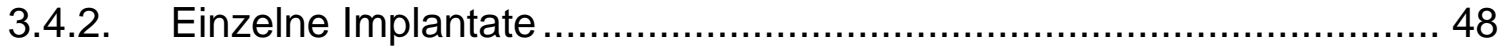

3.4.3. Cobb- und Kyphose-Winkel ..................................................... 50

3.5. Zusammenfassung der wichtigsten Ergebnisse .................................. 52

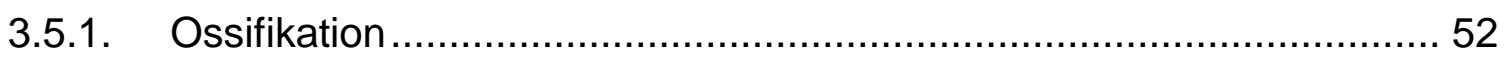

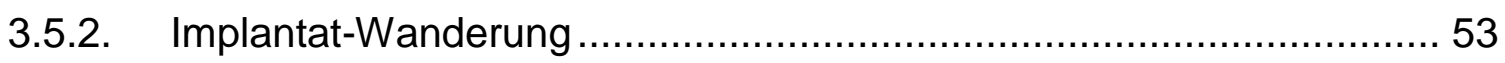




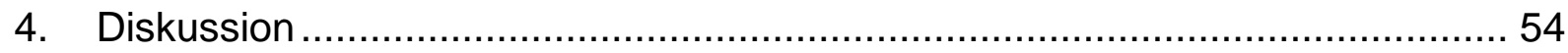

4.1. Aspekte der Behandlung von Skoliosen im Kindesalter .......................... 54

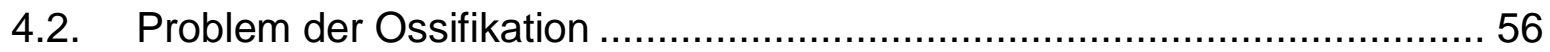

4.2.1. Inzidenz und Lokalisation ........................................................... 56

4.2.2. Zeitpunkt und Wahrscheinlichkeit ............................................... 59

4.2.3. Größe der Ossifikationen im Verlauf der Behandlung ........................ 61

4.2.4. Einflussgrößen auf die Ausbildung von Ossifikationen ....................... 61

4.2.5. Vergleich der Behandlungsdauer, der Operationsanzahl und der

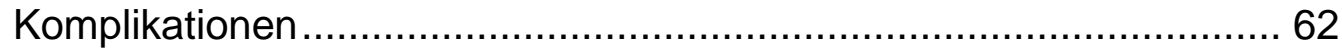

4.2.6. Folgen von entstandenen Ossifikationen......................................... 62

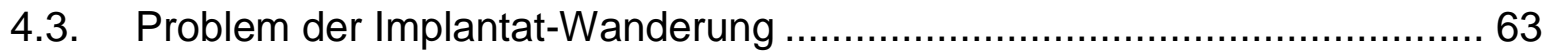

4.3.1. Implantat-Wanderung und Ossifikation............................................ 63

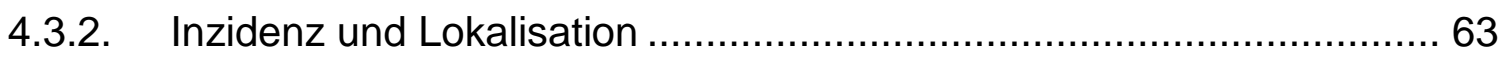

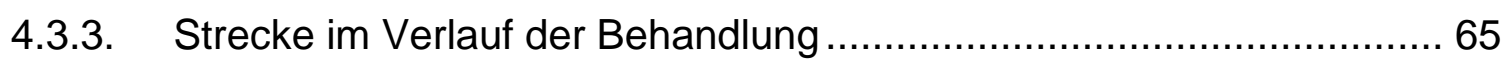

4.3.4. Einflussgrößen auf das Entstehen der Implantat-Wanderung ............. 66

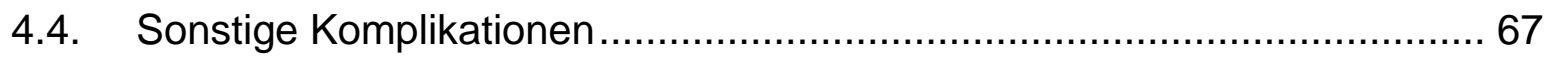

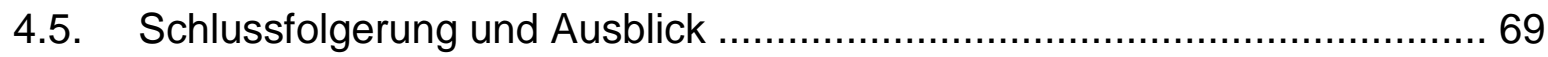

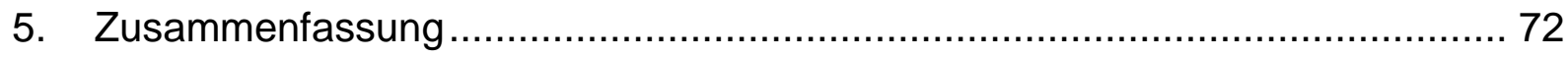

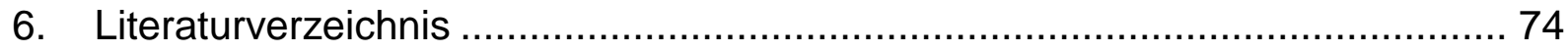




\section{Einleitung}

\subsection{Definition der Skoliose}

Die Skoliose ist definiert als eine seitliche Verkrümmung der Wirbelsäule mit einem Cobb-Winkel von mehr als $10^{\circ}$ in der koronaren Ebene und mit einer Torsion der Wirbelkörper und des Brustkorbs (Aebi 2005, Stokes 1994).

\section{2. Ätiologie und Epidemiologie der Skoliose}

Skoliosen lassen sich folgendermaßen einteilen:

- idiopathisch (infantil, juvenil, adoleszent): ca. 80\%

- neurogen: ca. $16 \%$

- kongenital: ca. 4\%.

\subsubsection{Idiopathische Skoliose}

Entsprechend dem heutigen Wissensstand geht man bei der idiopathischen Skoliose von einer multifaktoriellen Genese aus (Letellier et al. 2007):

- neurologische Ursachen

- muskuläre Dysbalancen

- biochemische Faktoren

- mechanische Störungen

- hormonelle Störungen

- neuroendokrine Störungen

- genetische Faktoren.

Die idiopathische Skoliose macht etwa $80 \%$ aller Skoliosen aus. In einer Untersuchung im schwedischen Malmö an Schulkindern zwischen sieben und 16 Jahren hatte sie bei Jungen eine Prävalenz von 0,5\% und bei Mädchen von 3,2\% (Willner und Udén 1982). Vermutlich durch degenerative Prozesse steigt die 
Prävalenz der idiopathischen Skoliose in der Bevölkerung bei 25- bis 74-Jährigen auf 8,3\% (Frauen 10,7\%, Männer 5,6\%) und bei 60 - bis 90 -Jährigen auf $68 \%$ (Frauen $73,1 \%$, Männer 56,5\%) an (Carter und Haynes 1987, Schwab et al. 2005). Eine Studie über 30 Jahre zeigte, dass $81 \%$ der idiopathischen Skoliosen den adoleszenten (elf Jahre bis Wachstumsabschluss), 11\% den juvenilen (vier bis zehn Jahre) und $8 \%$ den infantilen (null bis drei Jahre) Skoliosen zuzuordnen sind (Goldberg et al. 2008). Diese Verteilung scheint nur für Nordamerika zuzutreffen, denn in einer Studie mit Kindern im schottischen Edinburgh hatten 50\% der Patienten eine infantile Skoliose. Es wurde gezeigt, dass die Inzidenz der idiopathischen Skoliose in Bezug auf das Alter der Kinder zwei Höhepunkte hatte. Der erste Gipfel lag im Zeitraum der infantilen Skoliose (null bis drei Jahre), in der Zeit der juvenilen Skoliose (vier bis neun Jahre) traten wenige neue Fälle auf, bevor ein zweiter Anstieg der idiopathischen Skoliose in der Adoleszenz (zehn bis 18 Jahre) zu beobachten war (Wynne-Davies 1968). Zum besseren Vergleich der Skoliosen bildete Wynne-Davies die Kategorien der early-onset-Skoliose (unter acht Jahre) und der late-onset-Skoliose (acht Jahre und älter). Bei der early-onsetSkoliose waren im Verhältnis fünf Jungen und vier Mädchen, dagegen bei der lateonset-Skoliose sieben Mädchen und ein Junge betroffen (Wynne-Davies 1968). Basierend auf der Studie von Wynne-Davies ist die Inzidenz der early-onset-Skoliose 1,0/1000 und der late-onset-Skoliose 1,8/1000 Kinder. In beiden Kategorien waren Mädchen häufiger als Jungen betroffen. So wiesen Mädchen ein Neuauftreten von 1,4 bei early-onset-Skoliosen bzw. 3,9 bei late-onset-Skoliosen und Jungen eine Inzidenz von 0,6 bei early-onset-Skoliosen bzw. 0,3 bei late-onset-Skoliosen pro 1000 Patienten auf (James 1971).

Die Ergebnisse dieser Untersuchungen würden heutzutage etwas abweichen, da sich eine Definition durchgesetzt hat, in der das Auftreten einer Skoliose bis zum fünften Lebensjahr als early onset und das Auftreten ab dem sechsten Lebensjahr als late onset beschrieben wird (Akbarnia 2007).

\subsubsection{Infantile Skoliose}

Die infantile idiopathische Skoliose hat in den USA unter den idiopathischen Skoliosen die geringste Prävalenz, die zwischen $0,5 \%$ und $8 \%$ liegt. Jungen (57\%) sind etwas häufiger als Mädchen (43\%) betroffen (Goldberg et al. 2008, Moe und 
Kettleson 1970, Riseborough und Wynne-Davies 1973). Diese niedrige Prävalenz scheint nur für die USA zuzutreffen, da in Großbritannien eine Inzidenz von etwa $50 \%$ unter allen idiopathischen Skoliosen ermittelt wurde. In dieser Altersgruppe tritt die Skoliose verstärkt im ersten Lebensjahr auf und ist zu $88 \%$ linksthorakal gebogen (Wynne-Davies 1968). Bei Kindern mit zwei primären Kurven sind die thorakale Krümmung zur linken und die lumbale Kurve zur rechten Seite ausgeprägt (James 1970). In einer Studie von Ferreira et al. war die infantile idiopathische Skoliose zu $30 \%$ progressiv und zu $52 \%$ bildete sie sich zurück, die übrigen $18 \%$ wurden andersartig klassifiziert (Ferreira et al. 1972). Bei Connor et al. war sie zu $42 \%$ fortschreitend und zu 58\% rückläufig (Connor et al. 1987).

\subsubsection{Juvenile Skoliose}

In den USA ist die juvenile idiopathische Skoliose relativ selten unter den idiopathischen Skoliosen. So macht die juvenile idiopathische Skoliose zwischen $10,5 \%$ und $16 \%$ aller idiopathischen Skoliosen aus (Goldberg et al. 2008, Keiser und Shufflebarger 1976, Moe und Kettleson 1970, Riseborough und Wynne-Davies 1973). Das Verhältnis Mädchen:Jungen reicht von 1,6:1 über 3,1:1, 3,8:1 und 4,4:1 bis zu 6,3:1 (Goldberg et al. 2008, Mannherz et al. 1988, Riseborough und WynneDavies 1973, Robinson und McMaster 1996, Tolo und Gillespie 1978). In dieser Altersgruppe kommt es zu einem Übergang der Wirbelsäulenkrümmung von links- zu rechtsthorakal, so dass beide Formen ungefähr gleich oft vorliegen (Wynne-Davies 1968).

\subsubsection{Adoleszente Skoliose}

Die adoleszente idiopathische Skoliose hat unter allen idiopathischen Skoliosen eine Prävalenz von $81 \%$ und betrifft etwa in neun von zehn Fällen Mädchen (91\% zu 9\%) (Goldberg et al. 2008). Die Prävalenz der adoleszenten idiopathischen Skoliose beträgt etwa $2 \%$ bis $3 \%$ in der Altersgruppe der Zehn- bis 16-Jährigen, sofern der Cobb-Winkel mindestens $10^{\circ}$ aufweist. Je größer der Cobb-Winkel ist, desto geringer ist die Prävalenz der adoleszenten idiopathischen Skoliose. Beträgt die Kurve der Skoliose über $20^{\circ}$, so sind etwa $0,3 \%$ bis $0,5 \%$ dieser Kinder betroffen. Beträgt der Cobb-Winkel über $30^{\circ}$, so sind es $0,1 \%$ bis $0,3 \%$ und bei über $40^{\circ}$ sind es unter 
0,1\%. Bei Patienten mit einer kleinen Kurve von etwa $10^{\circ}$ weisen beide Geschlechter ungefähr die gleiche Prävalenz auf. Werden nur Patienten mit einem Cobb-Winkel über $30^{\circ}$ betrachtet, so sind zehnmal mehr Mädchen als Jungen betroffen (Weinstein 1999). In der Inzidenz und dem natürlichen Verlauf der adoleszenten idiopathischen Skoliose scheint es während einer jahrelangen Epoche keinen Wandel zu geben. Dies zeigte eine Längs- und Querschnittsstudie mit Kindern aus dem schwedischen Malmö über eine Dauer von 15 Jahren (Montgomery und Willner 1997). Die Skoliose ist in $90 \%$ der Fälle rechtsthorakal gebogen (Wynne-Davies 1968). Bei Patienten mit zwei primären Kurven sind die thorakale Krümmung zur rechten und die lumbale Kurve zur linken Seite ausgeprägt (James 1970).

\subsubsection{Neurogene Skoliose}

Als neurogene Skoliosen werden alle Skoliosen betitelt, bei denen eine neuromyopathische Grunderkrankung zugrunde liegt und der Patient nicht in der Lage ist, seine Wirbelsäue in der Achse zu halten. Die Ursachen hierfür können vielfältig sein, wie zum Beispiel die Duchenne-Muskeldystrophie, die infantile Zerebralparese, die spinale Muskelatrophie, die spastische Hemiparese, intraspinale Tumoren, die Myelomeningozele, Paraplegien oder ein Schädel-Hirn-Trauma (Brunner und Gebhard 2002). Im Folgenden werden die aufgrund einer spinalen Muskelatrophie oder einer Myelomeningozele auftretenden Skoliosen erläutert.

\subsubsection{Spinale Muskelatrophie und Skoliose}

Die spinale Muskelatrophie (SMA) ist eine genetisch bedingte neuromuskuläre Erkrankung mit weitreichender Muskelschwäche durch Degeneration der Vorderhornzellen des Rückenmarks (Schwentker und Gibson 1976). Sie wird normalerweise autosomal-rezessiv, in seltenen Fällen auch autosomal-dominant vererbt und betrifft etwas häufiger Jungen (Sucato 2007).

Die SMA wird nach dem erstmaligen Auftreten in vier Typen klassifiziert. Die SMA Typ I (Werdnig-Hoffmann) ist die schwerste Form und tritt in den ersten sechs Lebensmonaten, die SMA Typ II (intermediäre Form) zwischen dem sechsten und zwölften Lebensmonat, die SMA Typ III (Kugelberg-Welander) in der Kindheit ab dem zweiten Lebensjahr und die SMA Typ IV im Erwachsenenalter auf (Pearn 1980). 
Bei der SMA Typ I-III ergibt sich laut einer Studie, die in Nordostitalien erfolgte, eine Prävalenz von 7,8/100000 Lebendgeburten. Für die SMA Typ I allein beträgt sie 4,1/100000 Lebendgeburten (Mostacciuolo et al. 1992).

Das größte orthopädische Problem im Rahmen einer SMA ist die Skoliose. In einer Analyse von Evans et al. trat bei 87\% der Patienten eine Skoliose auf, wobei etwa die Hälfte der Kinder, die in der Lage waren zu laufen, und nahezu alle Kinder, die nicht eigenständig laufen konnten, eine Verkrümmung der Wirbelsäule bekamen. Je nach Schweregrad der SMA trat die Skoliose zu einem späteren Zeitpunkt auf (Evans et al. 1981). Bei Schwentker und Gibson hatten von 35 Patienten 19 (54\%) eine schwere Skoliose $\left(>60^{\circ}\right)$. Analog zur Studie von Evans et al. hatten Patienten, die nicht laufen konnten, früher und häufiger Skoliosen, die zudem einen höheren Schweregrad aufwiesen, als Kinder, die laufen konnten. Es bestand keine Beziehung zwischen der Konvexität der Kurve mit der Seite der größeren Muskelschwäche bei Patienten mit asymmetrischer Parese. Die Skoliose trat durchschnittlich zum ersten Mal im Alter von sieben Jahren auf (Schwentker und Gibson 1976). In einer Untersuchung von Riddick et al. bekamen Kinder im Mittel mit sechs Jahren eine Wirbelsäulendeformität (90\% Skoliosen). Skoliosen traten ab dem dritten Lebensjahr auf und schritten ohne Behandlung fast immer fort (Riddick et al. 1982).

Die Skoliose hat die für eine neuromuskulär bedingte Wirbelsäulendeformität typisch lang gezogene C-Form. In 90\% der Fälle sind die Skoliosen einbogig, von denen $80 \%$ thorakolumbal und $20 \%$ thorakal auftreten. Die Kurven sind in der Regel rechtsgebogen. Doppelbogige Kurven sind thorakal rechtsgebogen und lumbal linksgebogen. Die SMA-Skoliosen sind flexibler und schneller fortschreitend als idiopathische Skoliosen (Sucato 2007).

\subsubsection{Myelomeningozele und Skoliose}

Die Myelomeningozele (MMC) ist eine kongenitale Fehlbildung des Rückenmarks, das sich aufgrund einer Bogenverschlussstörung der Wirbelkörper samt umgebenden Rückenmarkshäuten in Form des Duralsacks darstellt (Bremer 2003).

Die Inzidenz der MMC ist je nach Land unterschiedlich. In Großbritannien ist sie etwa 2,5-3,0/1000 Geburten und in den USA 1,05/1000 Lebendgeburten, wobei hellhäutige Menschen 2,5mal häufiger betroffen sind als Afroamerikaner. In

Schweden beträgt die Inzidenz 0,72/1000, in Japan 0,1/1000 und in 
Westdeutschland 0,57/1000 Lebendgeburten (Lorber 1966, Lüder und Schulte 1989). Durch konsequente Folsäureprophylaxe konnte in den letzten Jahren die Inzidenz der MMC drastisch gesenkt werden (De Wals et al. 2007).

Neben dem Auftreten einer Skoliose besteht bei der MMC aufgrund der fehlenden posterioren Elemente und damit fehlenden dorsalen Zuggurtung sowohl das Problem der Instabilität der Wirbelsäule als auch der Entwicklung einer Kyphose. Dies führt in der Regel zu einer collapsing spine, die ein thoracic insufficiency syndrome (Kapitel 1.3.), schwere neurologische Komplikationen inklusive Hautulzera und Verlust der Gehfähigkeit hervorrufen kann (Flynn et al. 2011).

Marchesi et al. unterteilten die MMC-Skoliose in eine angeborene und eine sich entwickelnde Form. Die kongenitalen Kurven wiesen alle eine schnelle Progression bis zu einer Größe von mehr als $100^{\circ}$ auf. Die Kurven, die sich unter zehn Jahren entwickelten, nahmen schnell auf bis zu $70^{\circ}$ zu und solche, die sich nach dem zehnten Lebensjahr entwickelten, überschritten nie $30^{\circ}$ (Marchesi et al. 1991). In einer Studie in Hartford, Connecticut (USA), hatten 52\% der Patienten mit MMC eine Skoliose von mindestens $20^{\circ}$, die am häufigsten in der Lendenwirbelsäule ohne Bevorzugung einer Kurvenseite auftrat. Bestand noch keine Kurve bei Geburt, so lag das Durchschnittsalter bei Auftreten der Skoliose bei 9,6 Jahren. Etwas über die Hälfte der Kinder (58\%), die eine Skoliose entwickelten, bekamen diese vor dem neunten Lebensjahr und die übrigen (42\%) bis zum 15. Lebensjahr. Entstand bei den Patienten bis zu diesem Zeitpunkt keine Skoliose, wird dieser Zustand voraussichtlich bis zum Lebensende Bestand haben. Des Weiteren korrelierte die Inzidenz unmittelbar mit der motorischen Ebene und dem letzten intakten Wirbelbogen des Patienten. So ist das Risiko, eine Skoliose zu bekommen, größer, je höher die motorische Ebene (93\% thorakal, $8 \%$ sakral) bzw. der letzte intakte Wirbelbogen (89\% thorakal, $12 \%$ 4.-5. Lendenwirbel) liegt (Trivedi et al. 2002). In Westschweden bestand kein Unterschied in der Prävalenz der Skoliose im Hinblick darauf, ob ein Patient symmetrische oder asymmetrische neurologische Ausfälle hatte (Müller und Nordwall 1992).

In verschiedenen Studien wurden 7\%, 15\% bzw. 38\% der Skoliosen im Rahmen einer MMC als kongenital klassifiziert (Müller und Nordwall 1992, Piggott 1980, Samuelsson und Eklöf 1988). Dies setzt voraus, dass bereits bei Geburt eine abnorme Wirbelkörperentwicklung mit Wirbelkörperfehlbildungen vorlag (Lonstein 2000). 


\subsubsection{Kongenitale Skoliose}

Die Ätiologie und Epidemiologie lässt sich wie folgt zusammenfassen (Hell 2004):

Die kongenitale Skoliose wird durch Wirbelkörperanomalien hervorgerufen, die zu einer Wirbelsäulenverkrümmung mit unausgewogenem Längenwachstum der Wirbelsäule führen (McMaster und Ohtsuka 1982). Per definitionem liegen diese Wirbelkörperanomalien bereits bei Geburt vor (Lonstein 2000). In der Klassifikation nach MacEwen wird die kongenitale Skoliose auf Segmentationsstörungen, Formationsstörungen oder gemischte Störungen zurückgeführt (Winter 1973). Nur 1\% der kongenitalen Skoliosen ist hereditären Ursprungs (Connor et al. 1987). Am häufigsten ist eine multifaktorielle Genese der Grund der Fehlbildungen. Diese werden in Defekte der Embryonalzeit (bis 56 Tage nach Befruchtung) und der Fetalzeit (ab 57. Tag bis Geburt) unterteilt (Tsou et al. 1980). In Tierversuchen konnte gezeigt werden, dass durch Röntgenbestrahlung eine Halbwirbelanomalie und durch Kohlenmonoxid bei einer schwangeren Maus beim Nachkommen eine thorakale kongenitale Skoliose induziert wurde (Farley et al. 2001, Schmorl und Junghanns 1968).

Die genaue Prävalenz ist unbekannt, weil Wirbelkörperanomalien, die klinisch nicht auffallen, häufig nur zufällig entdeckt werden. In England wurde eine Untersuchung an einer Schule durchgeführt, in der von 1800 Kindern zwei an einer kongenitalen Skoliose litten. Dies wären in etwa 1\%o (Dickson et al. 1980). Die Kurven sind in der Regel sehr steif und daher schwierig zu korrigieren (McMaster und Ohtsuka 1982).

\subsection{Das thoracic insufficiency syndrome}

Viele Patienten weisen neben der Skoliose auch das Problem eines deformierten Thorax auf. Im Jahr 2003 definierten daher Campbell et al. das thoracic insufficiency syndrome (Thorax-Insuffizienz-Syndrom) als das Unvermögen des Thorax, eine adäquate Respiration bzw. normales Lungenwachstum zu gewährleisten (Campbell et al. 2003). Als Folge der Wirbelsäulen- und Thoraxdeformität bei Kindern entsteht eine Alteration der Lunge mit Verringerung der Lungenfunktion (Abb.1). Das Wachstum der Kinder verstärkt die Rotation bzw. die Krümmung der Wirbelsäule. Die Lunge wächst folglich aufgrund des Platzmangels im Brustkorb nicht altersgerecht mit (Campbell und Smith 2007). 


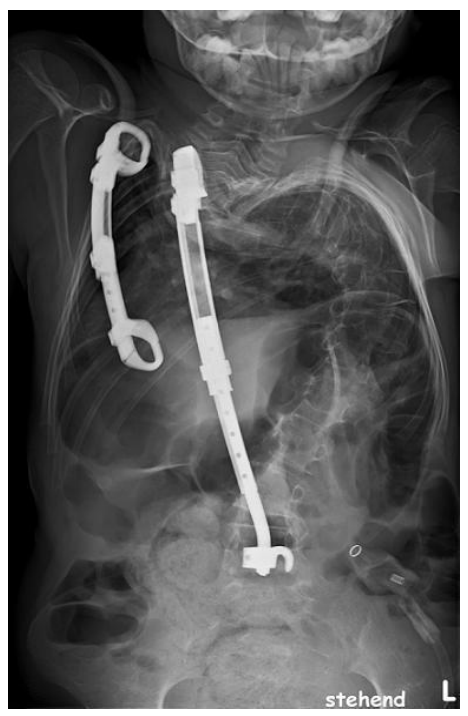

Die Diagnose des thoracic insufficiency syndrome besteht aus sechs Parametern. Hierzu gehören die Krankengeschichte des Patienten (z.B. wiederkehrende Pneumonien), die Klinik (z.B. Atemfrequenz, Brustumfang und -mobilität, Zyanose) und das Labor (z.B. Blutgase, Serumelektrolyte). Weitere Kategorien stellen das Röntgen (z.B. Skoliose, fusionierte Rippen), die Computertomografie (z.B. Verlust des Volumens einer Thoraxseite) als auch die Ventilations- und Perfusionslungenbildgebung bzw. der Lungenfunktionstest (z.B. Verlust der Vitalkapazität) dar. Die Diagnosefindung erfolgt interdisziplinär, indem diese sechs Kategorien von einem Kinderorthopäden, einem Kinderchirurgen und einem Kinderpulmologen beurteilt werden. Sie verteilen für jeden Aspekt mindestens einen Punkt (mild) und höchstens zehn (schwer). Die Wertungen der drei Spezialisten werden pro Parameter addiert und bei 20 oder mehr Punkten in einer Kategorie deutet dies auf ein thoracic insufficiency syndrome hin. Je mehr Kategorien 20 oder mehr Punkte aufweisen, desto schwerwiegender ist das thoracic insufficiency syndrome (Campbell und Smith 2007).

\subsection{Therapie der Skoliose}

Die Therapie der Skoliose ist stark abhängig von der Ätiologie der Erkrankung, vom Alter des Patienten bei Auftreten der Skoliose und vom Schweregrad der Deformität. Prinzipiell kann eine Behandlung konservativ oder operativ erfolgen (AWMF online 2009). 


\subsubsection{Konservativ}

Das primäre Ziel der konservativen Therapie der Skoliose ist, das Fortschreiten der Wirbelsäulenverkrümmung aufzuhalten. Des Weiteren sind die Verbesserung der Lungenfunktion (Vitalkapazität) und die Schmerztherapie von großer Wichtigkeit. Gegenwärtig stehen drei Standard-Therapieverfahren und zwei Alternativen zur Verfügung (Tab.1, AWMF online 2009, Weiß et al. 2006).

\begin{aligned} & Tab.1: Standard-Therapieve \\ & \hline - Korsetttherapie \\ & - Intensivrehabilitation \\ & - Physiotherapie \end{aligned}

Alternativen

Die Korsetttherapie bei der adoleszenten idiopathischen Skoliose bewirkt bei optimaler Passform und einer Tragedauer von mindestens 22 Stunden am Tag eine Verminderung des Fortschreitens der Krümmung und somit eine Verbesserung des natürlichen Skoliosenverlaufs (AWMF online 2009, Grivas et al. 2003, Nachemson und Peterson 1995). Zudem werden durch Korsetttherapie alle drei Ebenen erheblich korrigiert und die Anzahl an Operationen vermindert (Kotwicki et al. 2002, Rigo et al. 2003). Bei der neuromyopathischen Skoliose, die aufgrund der Grunderkrankung lebenslang fortschreiten kann, dient das Korsett neben der Korrektur der Deformität oder Fehlhaltung der funktionellen Stabilisierung des Rumpfes (Brunner und Gebhard 2002). Bei kongenitalen Skoliosen ist eine Korsetttherapie aufgrund der angeborenen Wirbelkörperfehlbildung nicht erfolgsversprechend (Lonstein 2000).

Die Intensivrehabilitation scheint sowohl auf Symptome und klinische Zeichen der Skoliose als auch auf den Verlauf der Wirbelsäulenverkrümmung effektiv positiven Einfluss zu nehmen (Weiß et al. 2003).

Bei der Physiotherapie steht die tägliche Anleitung zu einem Heimprogramm mit Trainingsformen zur Verbesserung der Rumpfmuskulatur und des Atemvolumens für die speziellen Feinheiten der Wirbelsäulenfehlbildung im Vordergrund (Weiß et al. 2003). Diese Anwendungen müssen durch Personal angeleitet werden, das spezifisch für diese konservativen Interventionsmethoden ausgebildet und zertifiziert ist (Weiß et al. 2006). 


\subsubsection{Operativ}

Bei der operativen Behandlung der Skoliose stehen ventrale und dorsale Verfahren bzw. die Kombination beider Verfahren zur Verfügung (Halm et al. 2009, Klöckner et al. 2000). Durch das verbleibende Wachstumspotenzial stellt die Wirbelsäulenchirurgie bei Kindern unter zwölf Jahren eine große Herausforderung dar.

\subsubsection{Dorsale Spondylodese}

In einem Übersichtsartikel von Halm et al. wurden die Charakteristika der dorsalen Spondylodese zusammengefasst (Halm et al. 2009):

Einen Meilenstein der dorsalen Wirbelsäulenchirurgie stellte das 1949 entwickelte Harrington-Instrumentarium dar. In den folgenden fünf Jahren wurde es $35 \mathrm{mal}$ modifiziert. Dieses Implantat wird am Anfangs- und Endpunkt der Wirbelsäulenkrümmung verankert und die Skoliose durch einen Stab eindimensional aufgerichtet (Harrington 1962). Dieses Verfahren war über 20 Jahre bis Ende der 1980er Jahre der Goldstandard.

In den 1980er Jahren wurde das Luque-Instrumentarium als erstes primärstabiles Implantat entwickelt. Dies ist ein segmental spinales Instrumentarium und wird heutzutage vor allem in der dritten Welt und bei neuromuskulären Skoliosen implantiert, da es eine kostengünstige Alternative zu anderen multisegmentalen Systemen darstellt (Luque 1982). Dieses Implantat hat sich aufgrund des erhöhten neurologischen Risikos, welches durch die sublaminare epidurale Drahtführung bedingt ist, zur Behandlung der idiopathischen Skoliose in der westlichen Welt nicht durchgesetzt.

Der allgemein gültige Standard in der dorsal instrumentierenden Skoliosechirurgie war lange Zeit das dreidimensional korrigierende Cotrel-Dubousset-Instrumentarium, das ebenfalls Mitte der 1980er Jahre entwickelt wurde. Dieses Implantat wird eingebracht, der Wirbelsäule angepasst, durch eine Drehung in die gewünschte sagittale Ebene befördert und folgend an der Wirbelsäule befestigt (Cotrel et al. 1988, Dubousset und Cotrel 1991).

In den letzten 15 Jahren wurden viele dorsale Doppelstabsysteme weiterentwickelt, die sich in drei Kategorien einteilen lassen: 
- ausschließlich hakengetragene Doppelstabsysteme (gegenwärtig weitgehend verlassen)

- ausschließlich pedikelschraubengetragene Doppelstabsysteme (gegenwärtig favorisiert)

- Hybridinstrumentarien (Kombination aus den beiden erstgenannten).

Derzeit gibt es einen Trend zur ausschließlich pedikelschraubengetragenen Spondylodese, da dieses System einen kürzeren Fusionsbezirk und exzellente Korrekturen auch höhergradiger rigider Skoliosen aufweist. Bei Anwendung dieser neu entwickelten Systeme ist eine Korrektur der Krümmung in einer Größenordnung von etwa 60\% zu erzielen (Hopf 2000).

Klassische Indikationen für die dorsale Spondylodese sind ausgeprägte Skoliosen mit noch stark vorhandenem Wachstumspotenzial bzw. zu erwartendem starken Fortschreiten der Krümmung, die sich konservativ nicht beherrschen lassen (Alici und Pinar 1992).

\subsubsection{Ventrale Verfahren}

In dem Übersichtsartikel von Halm et al. wurde über die ventralen Verfahren folgendes zusammengefasst (Halm et al. 2009):

Den Beginn der ventral instrumentierenden Skoliosechirurgie stellte das DwyerInstrumentarium dar, das die Kurve der Skoliose durch konvexseitige Kompression ausgleicht (Dwyer et al. 1969).

In den Jahren 1973/74 wurde die ventrale Derotationsspondylodese, international als Zielke-Instrumentarium bekannt, entwickelt (Zielke 1982). Diese galt über zwei Jahrzehnte als Goldstandard. Es war das erste unter Ausschöpfung aller technischen Möglichkeiten effektiv dreidimensional korrigierende System (Giehl et al. 1989). Allerdings hatte die ventrale Derotationsspondylodese vielfach Komplikationen wie Pseudarthrosenbildung, kraniale Schraubenausrisse und Stabbrüche zur Folge. Deshalb musste oft monatelang mit Korsett oder Rumpfgips nachbehandelt werden (Betz et al. 1999, Liljenqvist und Halm 1998).

So wurden primärstabile ventrale Implantate entwickelt, die vergleichbar gut korrigieren, eine signifikant niedrigere Komplikationsrate aufweisen und keiner Nachbehandlung mit Korsett bedürfen. Bei diesen Systemen ist eine Korrektur der 
Krümmung in einer Größenordnung von bis zu 75\% zu erreichen (Hopf 2000). Folgende Systeme werden unterschieden:

- primärstabile Einstabsysteme

- primärstabile Doppelstabsysteme

- Einstab- oder Doppelstabsysteme in Kombination mit interkorporellen Cages.

Klassische Indikationen für ventrale Verfahren sind strukturell einbogige lumbale, thorakolumbale (Lenke Typ 5) und thorakale Skoliosen (Lenke Typ 1). In Ausnahmefällen ist es auch möglich, eine doppelbogig thorakale Krümmung (Lenke Typ 2) auf diese Weise zu versorgen (Halm et al. 2009).

\subsubsection{Traditionelle Verfahren bei Kindern $<10$ Jahre}

Über die traditionellen Verfahren bei Kindern unter zehn Jahren fasste Hell folgendes zusammen (Hell 2004):

Bei Kindern unter zehn Jahren mit kongenitaler Skoliose scheitert eine konservative Therapie wie das Tragen eines Korsetts oder die Physiotherapie fast immer (Lonstein 2000). Deshalb wurden in der Vergangenheit möglichst früh operative Eingriffe vorgenommen, um die zum Teil sehr starke Kurvenprogression zu stoppen. Zur Auswahl standen ventrale und/oder dorsale Fusionen, Epiphyseodesen, Arthrodesen oder der Versuch, die Skoliose mit einem Harrington growing rod aufzudehnen (Andrew und Piggott 1985, Letts und Bobechko 1974, Marks et al. 1996, McMaster 1998, Moe et al. 1984, Rinsky et al. 1985, Tello 1994, Winter 1973, Winter 1981, Winter und Moe 1982, Winter et al. 1968). Diese operativen Optionen brachten aus verschiedenen Gründen nicht den gewünschten Therapieerfolg. Die Kinder hatten in der Folge zwar eine einigermaßen gutgeformte, aber dafür zu kurze Wirbelsäule mit kleinem Thorax und kleiner Lunge. Je ausgedehnter der Fusionsbezirk war, desto kürzer war die Wirbelsäule des ausgewachsenen Patienten mit verstärkten Lungenproblemen (thoracic insufficiency syndrome, Lungenversagen) als Folge mangelnder Lungensubstanz (Hell 2004, Hell et al. 2005 a).

Als Alternative stand nur die alleinige Observation der Kurvenprogression zur Verfügung. Beide Therapiemöglichkeiten konnten das Problem des zu geringen Lungenwachstums nicht beheben (Hell 2004). 
Um diese Problematik zu adressieren, wurden expandierende „mitwachsende“ Systeme für Kinder entwickelt. Das VEPTR (vertical expandable prosthetic titanium rib)-Implantat wird in Kapitel 1.5. erläutert. Weitere Verfahren stellen die unterschiedlich modifizierten growing-rod-Systeme dar (Akbarnia und Emans 2010, Yazici und Emans 2009).

\subsubsection{Expandierende „mitwachsende“ Systeme}

Ein Beispiel solcher growing-rod-Verfahren ist das USS-pediatric-Instrumentarium, bei dem das Implantat per Pedikelschrauben befestigt wird, wobei die knöcherne Fusion im Scheitel der Krümmung entfällt. So kann alle vier bis sechs Monate eine Distraktion des Doppelstabsystems (dual growing rod) erfolgen. Auf diese Weise wird die wachstumslenkende und weichteilschonende Stabilisierung der Wirbelsäule mit korrigierender Wirkung ermöglicht (Pfandlsteiner et al. 2010). Die heutigen Systeme basieren zum Großteil auf dem Konzept des Harrington growing rods (Harrington 1962).

Dieses Verfahren kann alternativ zum VEPTR-Instrumentarium angewandt werden. Postoperativ ist das Tragen einer Orthese für drei bis acht Monate notwendig (Pfandlsteiner et al. 2010). Ein anderes Doppelstabsystem, das pediatric-IsolaInstrumentarium, weist vergleichbare Ergebnisse des Längenwachstums auf (Akbarnia et al. 2005, Akbarnia et al. 2008). Eine Alternative stellt das Einstabsystem (single growing rod) dar. Es gibt unterschiedliche Ergebnisse, welches System die höhere Komplikationsrate aufweist. In einer Untersuchung von Thompson et al. verursachte der single growing rod weniger Komplikationen sowie eine bessere Balance in der Frontal- und Sagittalebene. Der Vorteil des dual growing rods lag in seiner Kontrolle und Stärke, so dass initial eine signifikant bessere Korrektur der Skoliose erreicht wurde. Diese wurde zudem konsequenter aufrechterhalten und damit ein stärkeres Wachstum der Wirbelsäule erzielt als beim single growing rod (Thompson et al. 2005). In Studien von Akbarnia et al. und Bess et al. hingegen wies das Implantieren des dual growing rods eine niedrigere Komplikationsrate auf (Akbarnia et al. 2005, Bess et al. 2010). Derzeit zeigt sich ein Trend zur vermehrten Anwendung der dual-growing-rod-Technik (Thompson et al. 2005). 
Ein Problem dieser an der Wirbelsäule verankerten wachstumslenkenden Systeme besteht darin, dass es häufig zu spontanen Wirbelfusionen kommt (Cahill et al. 2010, Yazici und Emans 2009).

\subsection{Das VEPTR (vertical expandable prosthetic titanium rib)- Implantat}

\subsubsection{Geschichte und Entwicklung}

1989 wurde der erste vertical expandable prosthetic titanium rib (VEPTR, Synthes® Spine Co., Paoli, Pa.) von Prof. Dr. med. Robert M. Campbell und Dr. med. Melvin Smith am Christus Santa Rosa Children's Hospital in San Antonio, Texas (USA), implantiert. Der Grund hierfür war, dass auf diese Weise für einen Jungen mit terminaler Lungeninsuffizienz aufgrund fehlender Rippen als ultima ratio ein neuartiger Therapieversuch gestartet wurde. Postoperativ entwickelte sich dieser Index-Patient überraschenderweise sehr gut (Campbell et al. 2003). Daher wurden das Implantat und die Operationsmethode weiterentwickelt.

Seit dem Jahr 2000 erfolgten in weiteren Kliniken in den USA Operationen dieser Art. In Europa wurde die erste VEPTR-Implantation im Mai 2002 im UniversitätsKinderspital beider Basel, Schweiz, durchgeführt (Hell et al. 2004). Im September 2004 wurde das VEPTR-Instrumentarium durch die CE-Marken-Zulassung in Europa offiziell als Therapieverfahren zugelassen. Weltweit wurden bisher grob geschätzt etwa 10000 Kinder mit dem VEPTR versorgt (Hell 2011).

\subsubsection{Instrumentarium}

Der vertical expandable prosthetic titanium rib (VEPTR) ist ein den Thorax stabilisierendes longitudinales Teleskop-Implantat aus Titan (Hell et al. 2004). Das VEPTR-Instrumentarium (Synthes ${ }^{\circledR}$ Spine Co., Paoli, Pa.) beinhaltet drei verschiedene Implantate (Abb.2-4, Campbell et al. 2004 a):

- $\quad$ Implantat „Rippe zu Rippe“

- Implantat „Rippe zu Lendenwirbelsäule“

- Implantat „Rippe zu Becken“. 
Abb.2:

Der vertical expandable prosthetic titanium rib (VEPTR) am Skelettmodell.

Am rechten Hemithorax ist medial ein Implantat „Rippe zu Lendenwirbelsäule“ und lateral ein Implantat „Rippe zu Rippe“ befestigt (aus Hell 2004, 12).

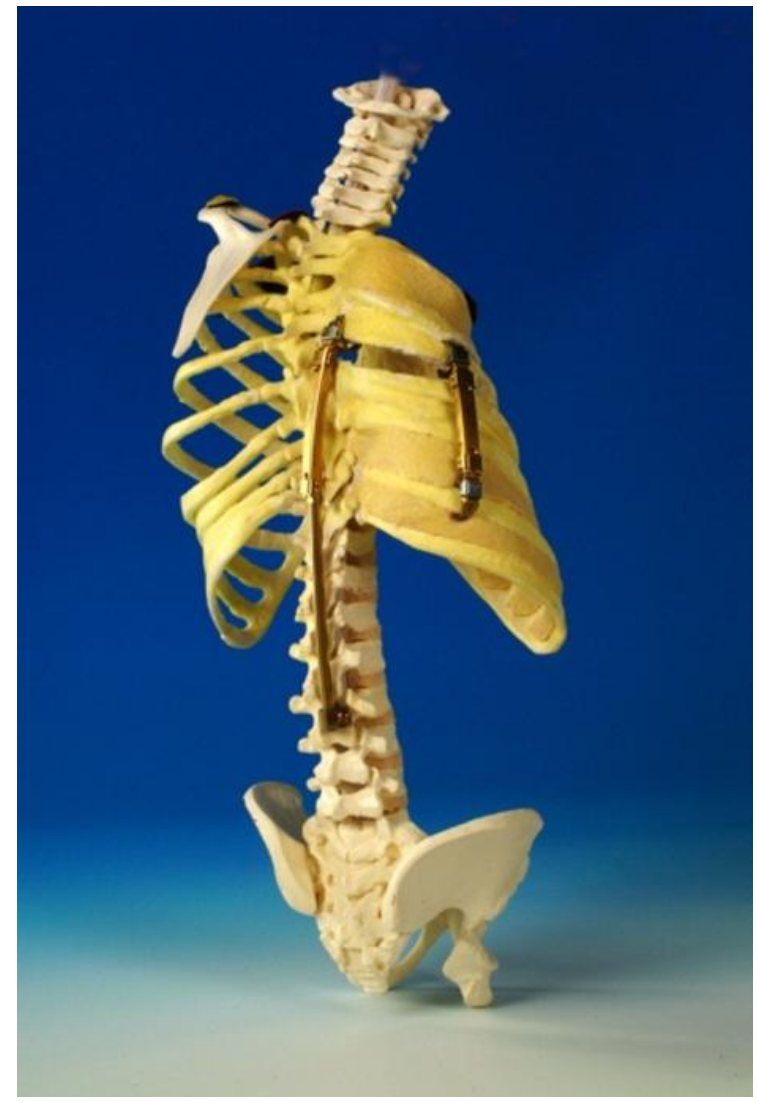

1. Hakenende

2. Hakensperre (blau)

3. Oberes Hakenende

4. Distraktionssperre (golden)

5. Rippenhülse

6. Unteres Hakenende

Abb.3:

Ein Implantat „Rippe zu Rippe“ zusammengesetzt (rechtes Bild) bzw. in seine Komponenten zerlegt (linkes Bild). Die Ringe werden jeweils um eine Rippe befestigt (aus Hell et al. 2004, 912, Bilder von Synthes ${ }^{\circledR}$, Stratec Medical, Oberdorf, Schweiz).

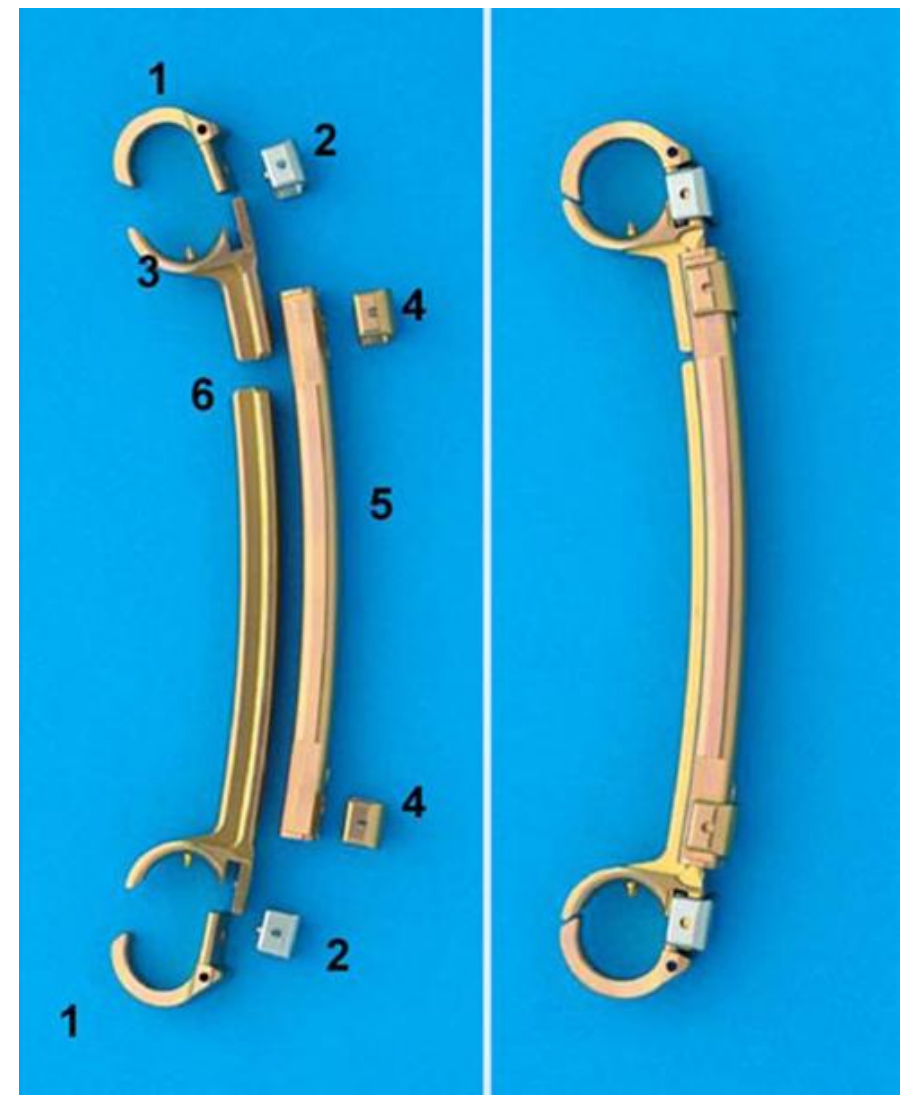


Abb.4:

Ein Implantat „Rippe zu Lendenwirbelsäule“ (linkes Bild) und ein Implantat „Rippe zu Becken“ (rechtes Bild). Der kraniale Ring wird jeweils um eine Rippe und der kaudale Haken an der thorakolumbalen Lamina des Wirbelbogens bzw. am Beckenkamm befestigt (aus Synthes ${ }^{\circledR}$ 2004, Stratec Medical, Oberdorf, Schweiz).

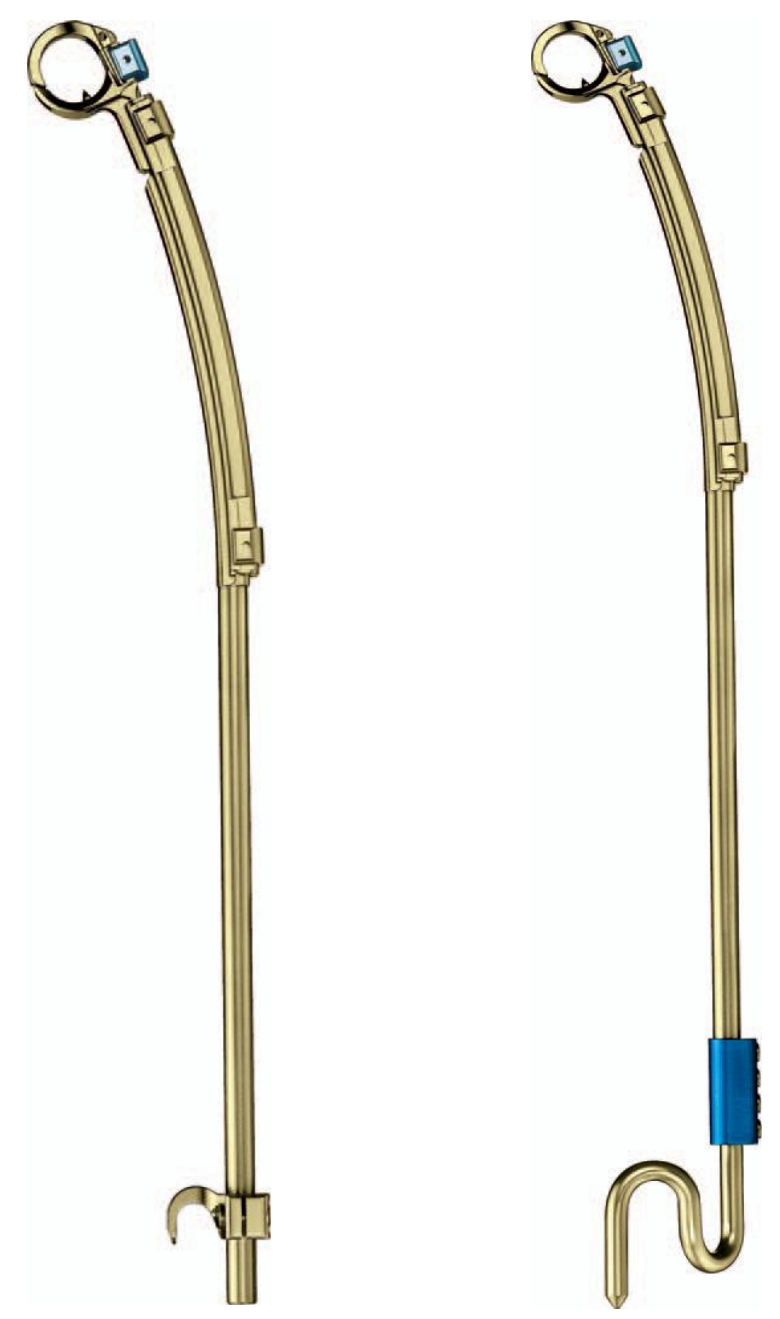

\subsubsection{Indikationen}

Die Indikation zur VEPTR-Behandlung ist bei skelettunreifen Patienten mit thoracic insufficiency syndrome und einer der folgenden Diagnosen gegeben (Campbell und Smith 2007):

- Flail chest syndrome

- Constrictive chest-wall syndrome, einschließlich:

- Rippenfusion und Skoliose

- Hypoplastic thorax syndrome, einschließlich:

- Ellis-van-Creveld-Syndrom

- Jeune-Syndrom

- Achondroplasie

- Jarcho-Levin-Syndrom

- Kongenitale oder neurogene progressive Skoliose ohne Rippenanomalie. 
Die Thorax- bzw. Wirbelsäulendeformitäten betreffen häufig alle drei Ebenen und führen vielfach zu einer volume depletion deformity, die in vier unterschiedliche Typen klassifiziert wird. Die Typen I und II verursachen eine einseitige Thoraxhypoplasie und die Typen IIla und IIlb eine globale Thoraxhypoplasie. An diesem Schema orientiert sich die Wahl des VEPTR-Systems (Campbell und Smith 2007):

- Typ I: Fehlende Rippen und Skoliose $\rightarrow$ „VEPTR (Becken) konkav einseitig“

- Typ II: Fusionierte Rippen und Skoliose $\rightarrow$ „VEPTR (Becken) konkav einseitig“

- Typ Illa: Verkürzter Thorax $\rightarrow$ „VEPTR beidseitig“

- Typ Illb: Eingeengter Thorax $\rightarrow$ „VEPTR beidseitig“

Die Thorax- bzw. Wirbelsäulendeformitäten, die nicht in dieses Schema passen, werden als exotisch beschrieben. Die verschiedenen VEPTR-Systeme werden in Kapitel 2.1.2. beschrieben.

\subsubsection{Operationsmethode}

Das Prinzip der Operation besteht darin, die Wirbelsäule durch das VEPTRInstrumentarium indirekt in ihrer Stellung zu korrigieren und den Thorax zu vergrößern, ohne dass ein Eingriff an der Wirbelsäule selbst bzw. eine Wirbelsäulenversteifung stattfindet. Die Operation sollte durch ein spezialisiertes Team erfolgen. Die Operationsmethode richtet sich nach dem Grundleiden und der Fehlbildung von Wirbelsäule und Rippen. Bei Kindern, die einseitig unsegmentierte Spangen und fusionierte Rippen bzw. ein thoracic insufficiency syndrome aufweisen, sollte eine Expansionsthorakoplastik mit öffnender Thorakotomie und VEPTRImplantation erfolgen. Sind die Rippen nicht miteinander verwachsen, so kann auf die Thorakotomie verzichtet werden (Abb.5, Akbarnia und Emans 2010, Campbell et al. 2004 a, Campbell et al. 2004 b, Hell et al. 2005 a, Hell et al. 2005 b).

Die Wahl der Implantatkonstellation hängt vom Typ der volume depletion deformity, der Grunderkrankung und dem Alter des Kindes ab. Ein Implantat „Rippe zu Rippe“ wird bei Kindern unter 18 Monaten verwendet, um dem Thorax eine bessere Stabilität zu verleihen. Dieses Implantat wird an einer kranialen und kaudalen Rippe befestigt. Durch die Distraktion der Rippen wird eine thorakale Skoliose indirekt 
aufgerichtet. Das Implantat „Rippe zu Lendenwirbelsäule“ wird verwendet, wenn die Skoliose tief thorakal oder am thorakolumbalen Übergang ihr größtes Ausmaß hat und das Kind älter als 18 Monate ist (Campbell und Smith 2007).
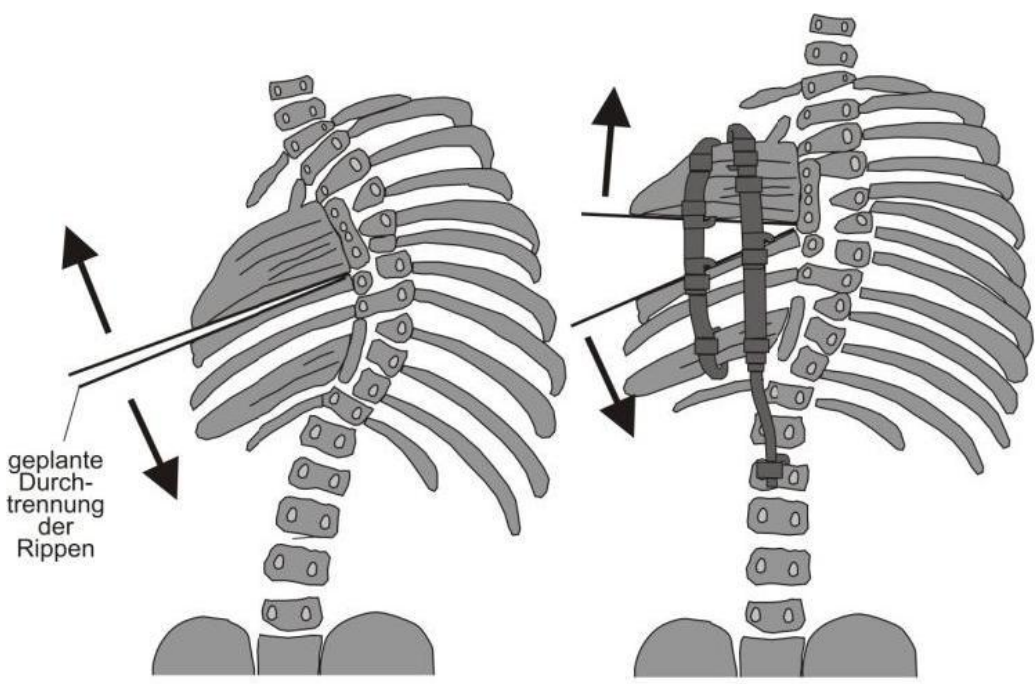

Öffnende Thorakotomie mit anschließender VEPTR-Implantation (aus Hell 2004, 13)

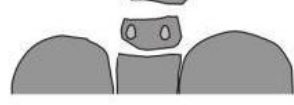

präop.

postop.

Bestehen Fehlbildungen an der Lendenwirbelsäule, wie zum Beispiel bei der Myelomeningozele, so wird das Implantat „Rippe zu Becken“ (Dunn-McCarthyHaken) verwendet. Bisher war das Implantat „Rippe zu Lendenwirbelsäule“ die primäre Option, weil bei Kleinkindern der Knochen des Wirbelbogens härter als der des Beckens ist. Bei neurogen bedingten Skoliosen wird hingegen das Implantat „Rippe zu Becken“ bevorzugt, da bei diesen Patienten aufgrund ihrer Lähmung keine Stabilität im Oberkörper vorhanden ist. Dies wird durch die Implantate „Rippe zu Becken“ am besten kompensiert, weil sowohl eine beidseitige Abstützung auf der gesamten Länge der Wirbelsäule gewährleistet werden kann als auch die Wirbelsäule selbst nicht berührt wird (Campbell und Smith 2007, Campbell et al. 2004 a, Flynn et al. 2011, Samdani et al. 2009, Smith 2011).

Die Operationsmethodik beinhaltet die Primär-Implantation sowie Verlängerungsoperationen, die alle sechs bis acht Monate bis zum Wachstumsabschluss erfolgen sollten. Das Alter bei Primär-Implantation liegt idealerweise zwischen sechs Monaten und drei Jahren, da sich hierdurch die Lungenfunktion in der Zukunft am besten entwickeln wird. Aufgrund dieses jungen Alters könnten bei einem Patienten im Verlauf der Behandlung über zwanzig 
Operationen durchgeführt werden. Findet die Primär-Implantation aus irgendeinem Grund zu einem späteren Zeitpunkt statt, so sollte sie vor dem achten Lebensjahr erfolgen, da die Lunge bis zu diesem Alter durch Multiplikation der Alveolarzellen zu wachsen scheint (Campbell et al. 2003, Campbell et al. 2004 a, Gollogly et al. 2004, Murray 1986, Waldhausen et al. 2007).

Das Ziel der Operationsmethode ist, dass die Stabilität, die Symmetrie und das Volumen des Thorax hergestellt und indirekt die Skoliose, der Rumpfüberhang, das Kopfalignement und die Rotation der Wirbelsäule korrigiert werden, ohne dass ein direkter Wirbelsäuleneingriff im Bereich der Deformität erfolgt (Campbell et al. 2004 b, Hell et al. 2004).

\subsection{Fragestellung}

In der vorliegenden Studie wurden Kinder und Jugendliche untersucht, die aufgrund von Skoliosen idiopathischer, neuromyopathischer oder kongenitaler Genese im Alter zwischen 1,33 und 18,25 Jahren eine primäre VEPTR-Implantation erhalten hatten. In der Literatur wurden spontane Wirbelfusionen und Ossifikationen nach operativen Verfahren wie z.B. der growing-rod-Technik im Kindesalter beschrieben (Cahill et al. 2010, Yazici und Emans 2009). In der vorliegenden Untersuchung wurden Ossifikationen am Brustkorb, der Wirbelsäule, am Becken oder entlang des Implantats im Verlauf der Behandlung analysiert. Die Inzidenz von Ossifikationen nach VEPTR-Implantation ist bisher unbekannt.

Anhand der Analyse von Röntgenaufnahmen wurden folgende Fragestellungen zu Ossifikationen nach VEPTR-Implantation behandelt:

- Wie häufig und an welcher Lokalisation treten radiologisch festzustellende Ossifikationen bei Kindern und Jugendlichen mit Skoliose auf, welche mittels VEPTR versorgt wurden?

- Zu welchem Zeitpunkt der Behandlung mit dem VEPTR entstehen Ossifikationen und wie groß ist die Wahrscheinlichkeit für Patienten, dass bei innen (keine) solche auftreten? 
- Wie groß sind die Ossifikationen und wie stark ist ihre Zunahme im Verlauf der Behandlung mit dem VEPTR?

- Lässt sich eine Korrelation vom Auftreten der Ossifikationen zum Alter bei der Primär-Implantation, Geschlecht, Grunderkrankung, Wahl der Implantatkonstellation, Cobb-Winkel prä bzw. post Primär-Implantation und/oder der Differenz des Cobb-Winkels prä und post Primär-Implantation feststellen?

- Hat das Auftreten von Ossifikationen einen vermehrten operativen Aufwand und/oder häufiger Komplikationen zur Folge?

Als weiterer Gegenstand der Analyse wurde die Implantat-Wanderung untersucht, da es sich hierbei wahrscheinlich um einen mittelbaren Risikofaktor für Ossifikationen handelt. In den bisherigen Studien wurden nur die symptomatischen, d.h. Operationen nach sich ziehenden, Implantat-Wanderungen untersucht. Im Rahmen dieser Untersuchung wurden daher folgende Fragestellungen verfolgt:

- Wie häufig und an welcher Lokalisation treten radiologisch festzustellende Implantat-Wanderungen bei Kindern und Jugendlichen mit Skoliose auf, welche mittels VEPTR versorgt wurden?

- Gibt es während der Behandlung mit dem VEPTR in Bezug auf die Strecke der Implantat-Wanderung Unterschiede zwischen den Implantaten?

- Gibt es in Bezug auf die Strecke der Implantat-Wanderung Unterschiede bei den Implantaten im Hinblick darauf, in welchem VEPTR-System sie verwendet wurden?

- Lässt sich eine Korrelation vom Auftreten der Implantat-Wanderungen zum Alter bei der Primär-Implantation, Geschlecht, Grunderkrankung, Wahl der Implantatkonstellation, Cobb-Winkel prä bzw. post Primär-Implantation und/oder der Differenz des Cobb-Winkels prä und post Primär-Implantation feststellen? 


\section{Material und Methoden}

\subsection{Patientenkollektiv}

In der vorliegenden Untersuchung wurden Kinder/Jugendliche $(n=57)$, die zu 54\% weiblich ( $n=31$ ) und zu 46\% männlich $(n=26)$ waren, analysiert, die in dem Zeitraum zwischen Mai 2002 und November 2010 eine VEPTR (vertical expandable prosthetic titanium rib)-Implantation erhalten hatten. Der Untersuchungszeitraum der retrospektiven Studie erstreckte sich von Mai 2002 bis Februar 2011.

Im Mittel wurden die Patienten über einen Zeitraum von 29,8 (1 bis 101) Monaten untersucht. Das durchschnittliche Alter der Kinder zur Zeit der primären VEPTRImplantation lag bei 7,66 Jahren. Das jüngste Kind war 1,33 und der älteste Jugendliche 18,25 Jahre alt.

\subsubsection{Grunderkrankungen}

Die Patienten ( $n=57$ ) wurden in folgenden Grunderkrankungen unterschieden:

- Spinale Muskelatrophie $(n=5 ; 9 \%)$

- Myelomeningozele $(n=10 ; 17 \%)$

- Kongenitale Skoliose $(n=9 ; 16 \%)$

- Sonstige Erkrankungen ( $n=33 ; 58 \%)$.

Aus dem Kollektiv $(n=57)$ waren vor der primären VEPTR-Implantation $47 \%$ der Patienten ( $n=27)$ gehfähig, 32\% der Kinder $(n=18)$ sitzfähig und 21\% der Patienten $(n=12)$ bettlägerig.

\subsubsection{Operative Versorgung}

Aufgrund verschiedener Kriterien (Grunderkrankung, Grad des Cobb-Winkels, Alter bei der Primär-Implantation, etc.) wurden bei den Patienten unterschiedliche VEPTRSysteme implantiert (Abb.6-8). 
- "VEPTR konkav einseitig"

$\rightarrow$ Das VEPTR-System wird auf der konkaven Seite der Skoliose implantiert, wobei das Implantat mit der Hauptlast die Lendenwirbelsäule mit einer Rippe verbindet.

$(n=29 ; 51 \%)$

- "VEPTR Becken konkav einseitig" $\rightarrow$ Das VEPTR-System wird auf der konkaven Seite der Skoliose implantiert. Es verbindet den Beckenkamm mit einer Rippe.

$(n=13 ; 23 \%)$

- "VEPTR beidseitig"

$\rightarrow$ Das VEPTR-System wird auf beiden Seiten der Skoliose implantiert. Die Implantate verbinden den Beckenkamm jeweils mit einer Rippe.

$(n=15 ; 26 \%)$
Abb.7: Pat. L. M.
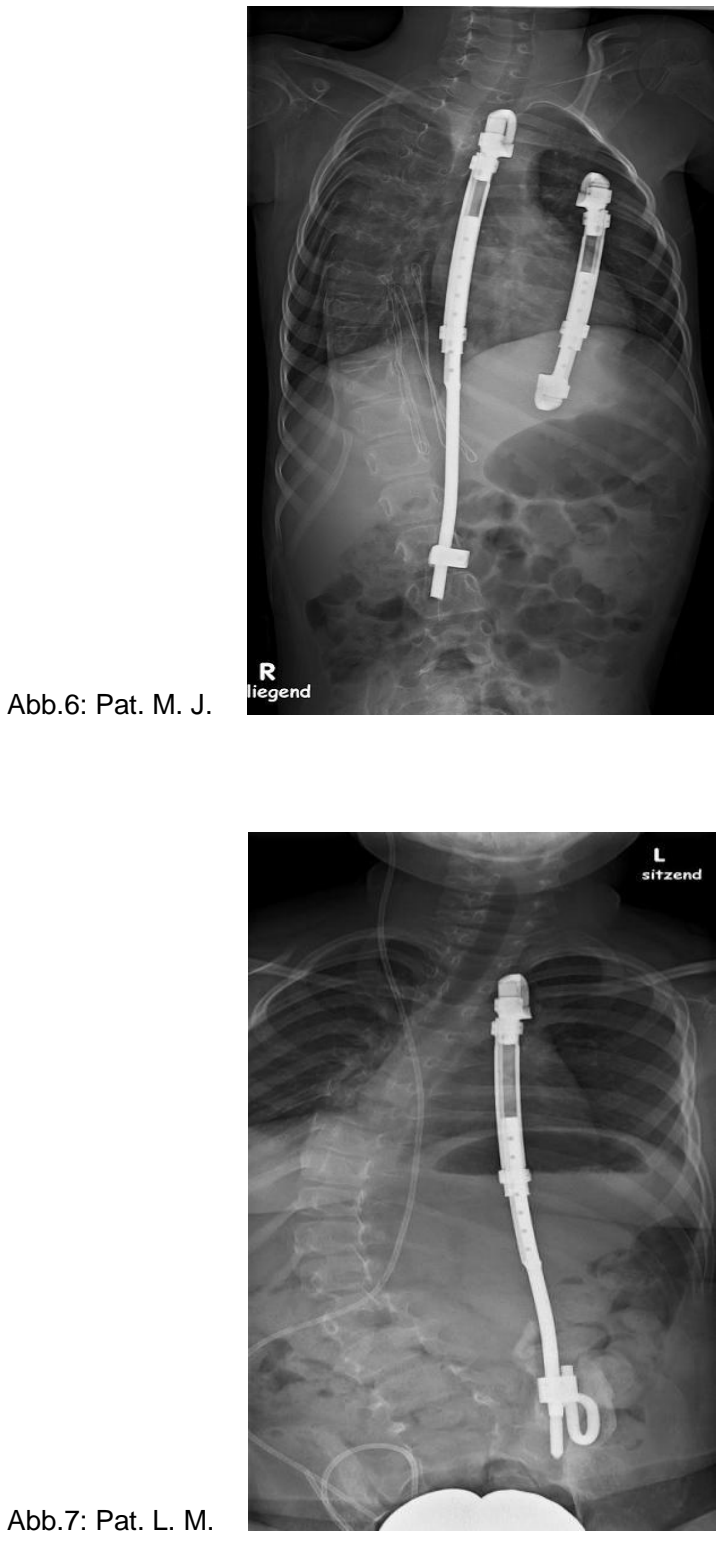

Abb.6: Pat. M. J.

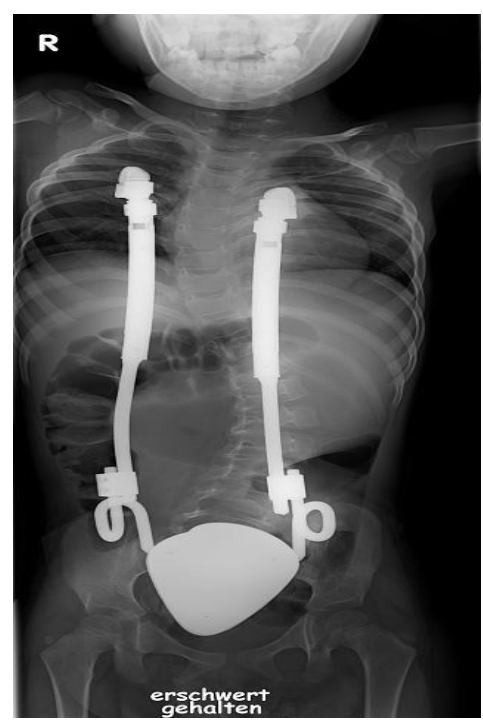


In dem Patientenkollektiv ( $n=57$ ) wurde 32mal das Implantat „Rippe zu Becken auf der konkaven Seite“, 31 mal das Implantat „Rippe zu LWS auf der konkaven Seite“, 23mal das Implantat „Rippe zu Rippe auf der konkaven Seite“ und 20mal das Implantat „Rippe zu Becken auf der konvexen Seite“ verwendet.

\subsubsection{Operativer Verlauf während der Nachuntersuchung}

Aus dem Kollektiv ( $n=57)$ waren 93\% der Patienten $(n=53)$ am Ende des Untersuchungszeitraums weiterhin in Behandlung mit dem VEPTR-Implantat. Die Kinder/Jugendlichen erhielten während ihrer Behandlung mit dem VEPTR im Mittel 5,9 ( 1 bis 19) Operationen. Von den Kindern $(n=57)$ erhielten 4\% $(n=2)$ in der Zeitspanne der Untersuchung nur eine primäre VEPTR-Implantation. Bei 7\% der Patienten $(n=4)$ wurde die Behandlung mit dem VEPTR-Implantat innerhalb des Untersuchungszeitraums durch eine Spondylodese beendet. Zu dem Zeitpunkt der Spondylodese waren die Patienten im Mittel 12,8 (10,4 bis 15,5) Jahre alt.

Im Rahmen der Nachuntersuchung wurden folgende Aspekte analysiert:

- Ossifikation

- Implantat-Wanderung

- Infektion am Implantat als Grund für einen vorzeitigen Implantat-Ausbau

- Implantat-Ausriss

- Implantat-Bruch

- Rippen-Spreizung nach Primär-Implantation

- nachträglicher Implantat-Einbau

- Umbau des VEPTR-Systems

- Implantat-Komponenten-Austausch

- Implantat-Umsetzung.

Die Untersuchungsweise dieser Aspekte wird in Kapitel 2.2. geschildert. 


\subsection{Untersuchung der Wirbelsäulenaufnahmen}

Die Patienten $(n=57)$ erhielten jeweils sowohl bei der primären VEPTR-Implantation als auch bei jeder Verlängerungsoperation prä- und postoperativ eine Röntgenaufnahme der Wirbelsäule. Diese Röntgenbilder wurden im anteriorenposterioren und im lateralen Strahlengang angefertigt. Insgesamt wurden 339 Operationen bei den Patienten ( $n=57)$ im Untersuchungszeitraum durchgeführt. In diesem Kollektiv konnte bei $96 \%$ der Kinder $(n=55)$ eine lückenlose radiologische Datenerhebung erfolgen. Bei $4 \%$ der Patienten $(n=2)$ fehlten Wirbelsäulenaufnahmen: Bei dem Jungen L. G. fehlten die Röntgenbilder sowohl der ersten als auch der vierten bis siebten Verlängerungsoperation. Bei dem Mädchen $P$. A. M. fehlten hingegen die Aufnahmen der primären VEPTR-Implantation und der ersten Verlängerungsoperation.

Aus dem Kollektiv $(n=57)$ erhielten $46 \%$ der Patienten $(n=26)$ ihre Wirbelsäulenaufnahme prä Primär-Implantation stehend, $31 \%$ der Kinder $(n=18)$ sitzend und $21 \%$ der Patienten $(n=12)$ liegend. Die Wirbelsäulenaufnahmen post Primär-Implantation erfolgten im Vergleich zum präoperativen Röntgenbild der Wirbelsäule bei $21 \%$ der Kinder $(n=12)$ in einer anderen Position (liegend; sitzend; stehend). Bei $2 \%$ der Patienten $(n=1)$ fehlten die prä- und postoperativen Wirbelsäulenaufnahmen der Primär-Implantation und waren somit nicht beurteilbar.

Auf 1328 Röntgenbildern wurden die radiologischen Aspekte/Komplikationen analysiert, die in drei Gruppen gegliedert wurden:

- Ossifikation

- Implantat-Wanderung

- Sonstige Komplikationen und radiologische Parameter (Cobb-Winkel prä und post Primär-Implantation, Kyphose-Winkel prä und post Primär-Implantation, nachträglicher Implantat-Einbau, Implantat-Ausbau, Implantat-Bruch, Implantat-Ausriss, Implantat-Umsetzung, ImplantatKomponenten-Austausch, Rippen-Spreizung nach Primär-Implantation).

Die Röntgenbilder wurden mittels standardisierter Röntgentechnik erstellt. Innerhalb des Untersuchungszeitraums wurden die Aufnahmen bis Dezember 2006 
konventionell erstellt. Seit dem Jahr 2007 lagen die Röntgenaufnahmen in digitalisierter Form vor.

\section{Hard- und Software}

Als Hardware diente der Computer Optiplex 745 der Firma DELL mit dem entsprechenden Bildschirm für die digitalisierten Röntgenbilder und der Röntgenbildbetrachter der Firma Planilux für die konventionellen Röntgenaufnahmen.

Als Software wurde zur Datenerhebung der digitalisierten Röntgenbilder das digitale Verarbeitungsprogramm Centricity ${ }^{\mathrm{TM}}$ und der konventionellen Röntgenaufnahmen ein Geometriedreieck verwendet.

\subsubsection{Ossifikation}

Bei jeder Röntgenaufnahme wurde untersucht, ob eine Ossifikation entstanden war oder sich eine bereits bestehende Verknöcherung im Hinblick auf die Größe verändert hatte. Hierbei wurde hinsichtlich der Lokalisation der Ossifikation unterschieden. Zudem wurde beschrieben, an welchem VEPTR-System die Ossifikation aufgetreten war. Zwischen folgenden Lokalisationen wurde unterschieden:

- Rippen (Implantat „Rippe zu Becken“, Implantat „Rippe zu LWS“, Implantat „Rippe zu Rippe“)

- Lendenwirbelsäule (Implantat „Rippe zu LWS“)

- Beckenkamm (Implantat „Rippe zu Becken“).

Ferner wurde untersucht, inwiefern sich die Ossifikation im Verlauf entwickelt hatte (Abb.9). Die Größe der Ossifikation wurde in $\mathrm{mm}^{2}$ gemessen. Dies erfolgte, indem zuerst die Länge der Verknöcherung gemessen, anschließend im rechten Winkel die durchschnittliche Breite geschätzt und diese wiederum gemessen wurde. Diese Messung erfolgte bei den digitalisierten Röntgenaufnahmen mit einer Funktion des Verarbeitungsprogramms Centricity ${ }^{\mathrm{TM}}$ und bei den konventionellen Röntgenbildern mittels Geometriedreieck. 
Abb.9:

Ossifikation (rot markiert) im Beckenbereich bei Patient J. L. im Februar 2010 (linkes Bild) und im November 2010 (rechtes Bild). In diesem Fall zeigte sich, dass die Ossifikation innerhalb der neun Monate Behandlung geringfügig gewachsen war.
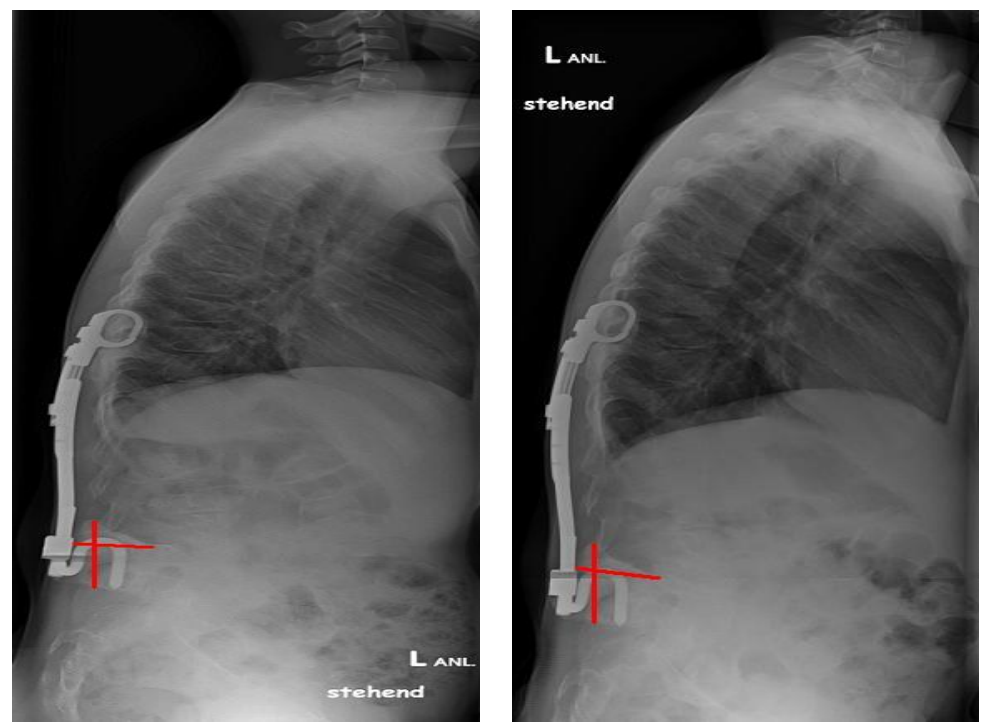

Die Ossifikation wurde an den verschiedenen Lokalisationen jeweils in der Ebene untersucht, in der sie am besten dargestellt bzw. ausgemessen werden konnte. Im Behandlungsverlauf wurde pro Patient durchgehend die gleiche Ebene analysiert. Im Bereich der Lendenwirbelsäule wurde die Ossifikation auf den Röntgenaufnahmen mit lateralem und am Beckenkamm mit anteriorem-posteriorem Strahlengang ausgemessen. Die Ossifikation im Rippenbereich wurde bei einem Patienten in der lateralen und bei zwei Kindern in der anterioren-posterioren Ebene untersucht.

\subsubsection{Implantat-Wanderung}

Auf jeder Röntgenaufnahme wurde untersucht, ob ein Implantat gewandert war. War dies der Fall, so wurde im Folgenden die Strecke der Wanderung gemessen. Hierbei wurde sowohl zwischen VEPTR-System als auch Lokalisation der Wanderung unterschieden. Folgende anatomische Regionen wurden betrachtet:

- Rippen (Implantat „Rippe zu Becken“, Implantat „Rippe zu LWS“, Implantat „Rippe zu Rippe“)

- Lendenwirbelsäule (Implantat „Rippe zu LWS“)

- Beckenkamm (Implantat „Rippe zu Becken“).

Die Implantat-Wanderung wurde dem kranialen oder kaudalen Ende eines Implantats zugeordnet. Ein präoperatives Röntgenbild wurde jeweils mit einem postoperativen verglichen, sodass festgestellt werden konnte, ob die Operation eine Wanderung des 
Implantats bewirkt hatte. Analog dazu wurde jeweils die postoperative mit der nachfolgenden präoperativen Aufnahme verglichen, um festzustellen, ob eine Implantat-Wanderung während des Operationsintervalls stattgefunden hatte. Gemessen wurde die jeweilige Wanderung in $\mathrm{mm}$. Die Durchführung dieser Messung erfolgte bei den digitalisierten Bildern mit dem Verarbeitungsprogramm Centricity ${ }^{\mathrm{TM}}$, indem auf dem Bildschirm zwei Aufnahmen parallel dargestellt und miteinander verglichen wurden. Aufgrund der unterschiedlichen Projektionen jedes einzelnen Röntgenbildes wurde die Implantat-Wanderung anhand fester Strukturen, die jeweils sicher identifiziert werden konnten, abgeschätzt. Hierfür diente zum Beispiel eine Rippe oder ein Lendenwirbelkörper. Der Lokalisationsunterschied des Implantatendes auf den zu vergleichenden Aufnahmen wurde gemessen (Abb.10). Die konventionellen Aufnahmen wurden auf dem Röntgenbildbetrachter nebeneinander aufgehängt und auf gleiche Weise mit einem Geometriedreieck ausgemessen.

Abb.10:

Implantat-Wanderung (rot markiert auf dem rechten Bild) an der kaudalen Verankerung des Implantats „Rippe zu LWS“ bei Patientin E. G.. Als Orientierung für die Messung der Strecke der Implantat-Wanderung dienten in diesem Fall die Dornfortsätze der Lendenwirbelkörper.
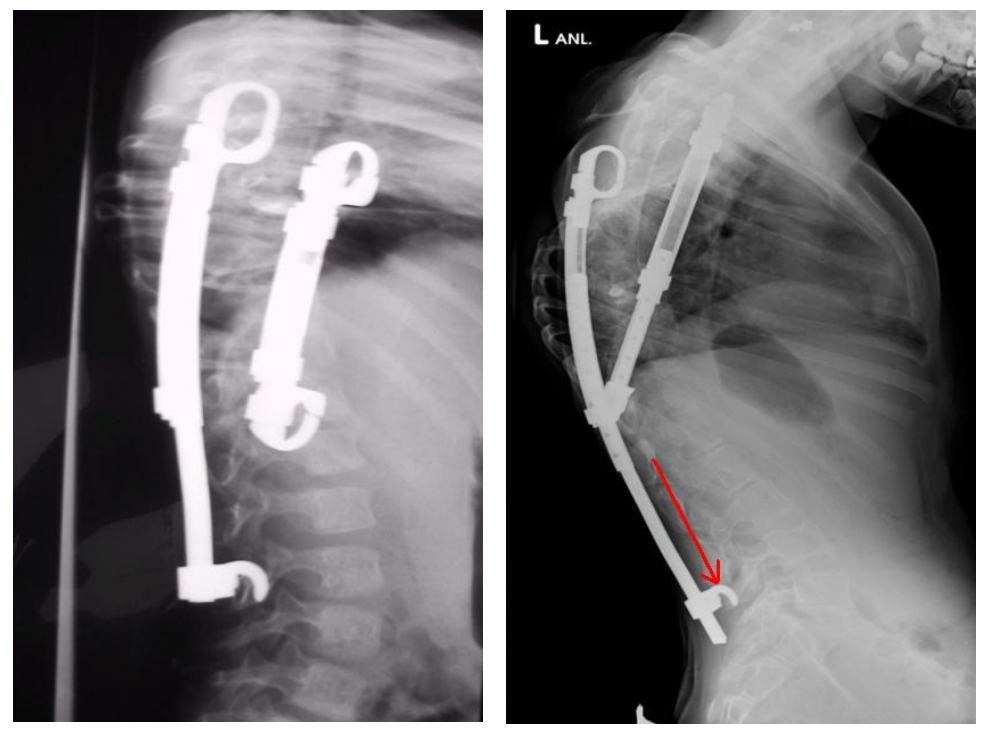

\subsubsection{Sonstige Komplikationen/radiologische Parameter}

Alle Röntgenaufnahmen wurden auf sonstige Komplikationen/radiologische Parameter untersucht. Dabei wurde folgendes analysiert:

- VEPTR-System

- Cobb-Winkel prä und post Primär-Implantation

- Kyphose-Winkel prä und post Primär-Implantation 
- Rippen-Spreizung nach Primär-Implantation

- Implantat-Bruch

- Implantat-Ausriss

- Implantat-Ausbau

- nachträglicher Implantat-Einbau

- Implantat-Umsetzung

- Implantat-Komponenten-Austausch.

Auf jeder Röntgenaufnahme wurde untersucht, ob diese Aspekte auftraten. Wenn dies der Fall war, so wurde die Lokalisation notiert. Bei allen Aspekten, die unmittelbar das Implantat betrafen, wurde zusätzlich betrachtet, welches Implantat des jeweiligen VEPTR-Systems betroffen war. Die digitalisierten Röntgenbilder wurden mit dem Verarbeitungsprogramm Centricity ${ }^{\mathrm{TM}}$ und die konventionellen Aufnahmen auf dem Röntgenbildbetrachter dargestellt. Die Untersuchung sonstiger Komplikationen/radiologischer Parameter wurde analog zur Messung der ImplantatWanderung durchgeführt, indem zwei aufeinanderfolgende Bilder parallel auf dem Bildschirm/Röntgenbildbetrachter verglichen wurden. Der Cobb- bzw. KyphoseWinkel der Skoliose wurde nach standardisierter Technik ausgemessen.

\subsubsection{Einflussgrößen auf die radiologischen Parameter}

Bezüglich der radiologischen Parameter wurden folgende Einflussgrößen analysiert:

- Geschlecht

- Grunderkrankung

- VEPTR-System

- Anzahl der Operationen

- Dauer der Behandlungszeit in Monaten

- Alter bei der Primär-Implantation

- Cobb-Winkel prä bzw. post Primär-Implantation

- Differenz der Cobb-Winkel prä und post Primär-Implantation

- Kyphose-Winkel prä und post Primär-Implantation. 
Das VEPTR-System, der Cobb- und Kyphose-Winkel prä bzw. post PrimärImplantation stellten sowohl radiologische Parameter als auch vermeintliche Einflussgrößen in Bezug auf die Komplikationen dar.

Die Einflussgrößen wurden dahingehend analysiert, inwiefern eine Korrelation mit den Ossifikationen, den Implantat-Wanderungen und den sonstigen Komplikationen/ radiologischen Parametern bestand.

Neben der Skoliose wiesen vier Patienten eine Hyperkyphose auf. Aufgrund dieser geringen Fallzahl erfolgte mit der Einflussgröße Kyphose-Winkel prä und post Primär-Implantation eine Darstellung der deskriptiven Werte ohne weitere Auswertung.

\subsection{Statistische Auswertung der Komplikationen/radiologischen Parameter}

Die statistische Auswertung der Komplikationen/radiologischen Parameter wurde mit dem Statistikprogramm statistica 9.0 durchgeführt. Diese erfolgte in den folgenden drei Kategorien:

- Ossifikation

- Implantat-Wanderung

- Sonstige Komplikationen/radiologische Parameter.

Das Signifikanzniveau wurde als $p<0,05$ definiert. Die Auswertung wurde mit folgenden Tests durchgeführt:

- Chi-Quadrat-Test

- student t-test für unverbundene Stichproben

- student $t$-test für verbundene Stichproben

- logistische Regression

- Kovarianzanalyse (ANCOVA).

Zur Veranschaulichung einiger wichtiger Ergebnisse wurden Histogramme, grafische Zeitverläufe, Mittelwertdiagramme und Kaplan-Meyer-Kurven verwendet. 


\section{Ergebnisse}

\subsection{Ossifikation}

\subsubsection{Inzidenz und Lokalisation}

In dem auszuwertenden Patientenkollektiv $(n=54)$, in dem 4\% $(n=2)$ nur eine PrimärImplantation erhalten hatten, traten bei $24 \%$ der Patienten $(n=13)$ Ossifikationen auf. Diese erschienen an folgenden Lokalisationen:

- $23 \%$ im Rippenbereich $(n=3)$

- $54 \%$ im Lendenwirbelsäulenbereich $(n=7)$

- $23 \%$ im Beckenbereich ( $n=3$; konkave Seite $100 \%$ ).

Bei der Analyse der Ossifikationen bezogen auf die einzelnen Implantate war die Verteilung folgendermaßen:

- $26 \%(n=8)$ der Implantate „Rippe zu LWS auf der konkaven Seite“ ( $n=31)$

- $88 \%$ kaudal $(n=7)$

- $12 \%$ kranial $(n=1)$

- $4 \%(n=1)$ der Implantate „Rippe zu Rippe auf der konkaven Seite“ ( $n=23)$

- $100 \%$ im Gesamten $(n=1)$

- $13 \%(n=4)$ der Implantate „Rippe zu Becken auf der konkaven Seite“ ( $n=32)$

- $75 \%$ kaudal $(n=3)$

- $25 \%$ kranial $(n=1)$

- $0 \%(n=0)$ der Implantate „Rippe zu Becken auf der konvexen Seite“ $(n=20)$.

Bei der Analyse der Ossifikationen bezogen auf die anatomische Lokalisation der Implantate war die Verteilung folgendermaßen:

- $3 \%(n=3)$ der Implantate im Rippenbereich $(n=106)$

- $23 \%(n=7)$ der Implantate im Lendenwirbelsäulenbereich $(n=31)$

- $6 \%(n=3)$ der Implantate im Beckenbereich ( $n=52$; konkave Seite $100 \%$ ). 


\subsubsection{Zeitpunkt und Wahrscheinlichkeit}

Im vorliegenden Nachuntersuchungszeitraum wurde anhand des Patientenkollektivs analysiert, wie viele Patienten prozentual im Verlauf eine Ossifikation bekamen:

- $11 \%$ in den ersten zwölf Monaten der Behandlung

- $12 \%$ ab dem 13. bis einschließlich 24. Monat der Behandlung

- $12 \%$ ab dem 25. bis einschließlich 48. Monat der Behandlung

- $14 \%>48$ Monate Behandlungsdauer ( $n=7)$.

Werden die ersten 24 Monate der Behandlung als Zeitraum zusammengefasst, so bekamen 19\% der Patienten eine Ossifikation. Analog wurde untersucht bei welcher Anzahl an Operationen Ossifikationen prozentual auftraten:

- $13 \%$ ab der ersten bis einschließlich dritten Operation

- $11 \%$ ab der vierten bis einschließlich sechsten Operation

- $21 \%$ ab der siebten bis einschließlich neunten Operation

- $0 \%>$ neun Operationen $(n=4)$.

Anhand des Patientenkollektivs wurden die absoluten Zahlen des erstmaligen Auftretens einer Ossifikation ermittelt (Abb.11, Abb.12). Zudem wurde analysiert, in welcher Zeitspanne Patienten ohne Ossifikation blieben (Abb.13, Abb.14).

Abb.11:

Darstellung des erstmaligen Auftretens

einer Ossifikation während der Behandlungsdauer in Monaten:

Erstmaliges Auftreten einer Ossifikation mit Schwerpunkt zwischen dem sechsten und zwölften Monat der Behandlung.

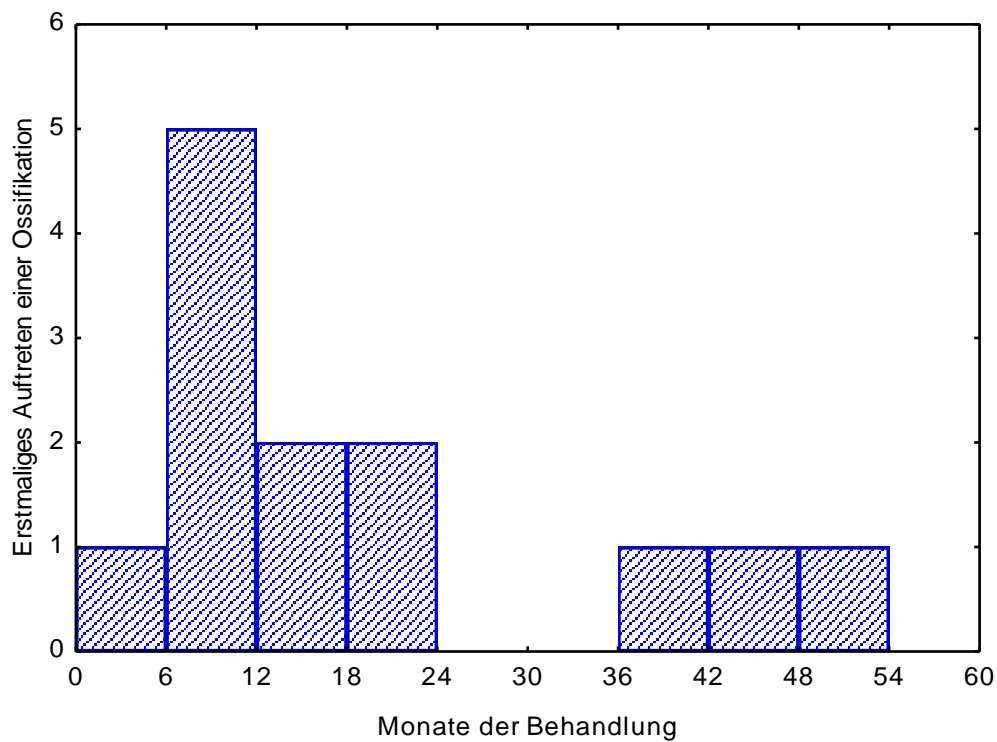


Abb.12:

Darstellung des erstmaligen Auftretens einer Ossifikation bei unterschiedlicher Anzahl an Operationen:

Erstmaliges Auftreten einer Ossifikation mit Schwerpunkt ab der zweiten Operation bis einschließlich der vierten.

Abb.13:

Darstellung der Wahrscheinlichkeit des Patienten nicht ossifiziert zu sein während der Behandlungsdauer in Monaten:

Nach 21 Monaten waren 74\% der Patienten nicht ossifiziert.

Nach 53 Monaten blieben 52\% der Patienten ohne Ossifikation.

Abb.14:

Darstellung der Wahrscheinlichkeit des Patienten nicht ossifiziert zu sein bei unterschiedlicher Anzahl an Operationen: Nach vier Operationen waren $75 \%$ der Patienten nicht ossifiziert.

Nach neun Operationen blieben 54\% der Patienten ohne Ossifikation.
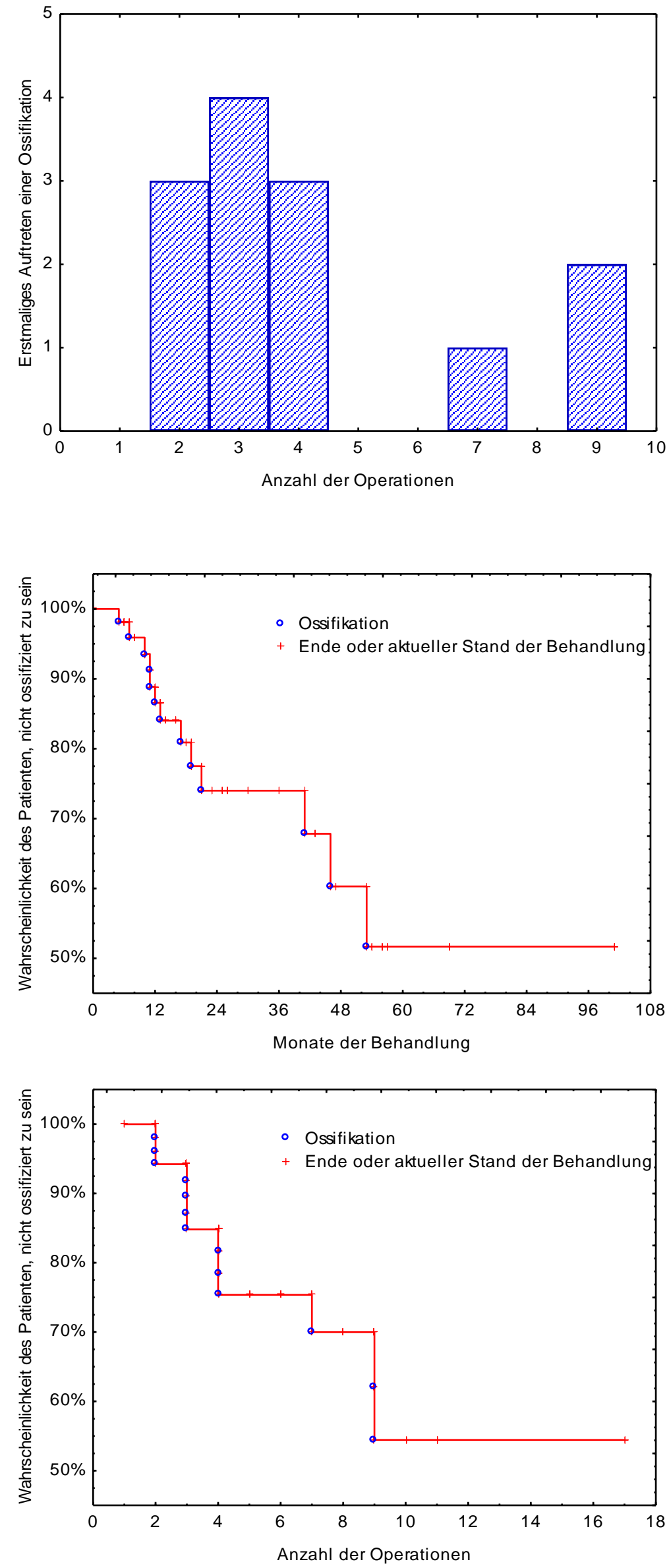
Des Weiteren wurde anhand des Patientenkollektivs berechnet, wie hoch vor Beginn der Implantation eines VEPTR-Instrumentariums die Wahrscheinlichkeit für einen Patienten war, eine Ossifikation im Verlauf der Behandlung zu bekommen:

- $11 \%$ in den ersten zwölf Monaten

- $10 \%$ ab dem 13. bis einschließlich 24. Monat

- $10 \%$ ab dem 25. bis einschließlich 48. Monat

- $10 \%$ bei > 48 Monaten Behandlungsdauer $(n=7)$.

Analog dazu lag die berechnete Wahrscheinlichkeit in Bezug auf die Anzahl der Operationen bei:

- $13 \%$ ab der ersten bis einschließlich dritten Operation

- $10 \%$ ab der vierten bis einschließlich sechsten Operation

- $17 \%$ ab der siebten bis einschließlich neunten Operation

- $0 \%>$ neun Operationen $(n=4)$.

\subsubsection{Größe der Ossifikationen im Verlauf der Behandlung}

Anhand des Patientenkollektivs wurde analysiert, inwiefern sich die Größe der Ossifikationen im Verlauf veränderte (Tab.2).

\begin{tabular}{l|c|c} 
Tab.2: & Größe in $\mathrm{mm}^{2}$ & Größenzunahme in Prozent \\
\hline $\begin{array}{l}\text { Größe bei erstmaligem } \\
\text { Auftreten }\end{array}$ & 400 (48 bis 1664) & \\
\hline $\begin{array}{l}\text { Größe bei der ersten } \\
\text { Verlängerungsoperation }\end{array}$ & 574 (51 bis 2624) & 44 (0 bis 170) \\
nach erstmaligem Auftreten & & \\
\hline $\begin{array}{l}\text { Größe nach im Mittel 3,9 } \\
\text { (1 bis 10) } \\
\text { Verlängerungsoperationen } \\
\text { nach erstmaligem Auftreten }\end{array}$ & 1071 (75 bis 3399) & 168 (19 bis 1590) \\
\end{tabular}


Bei $46 \%$ der Patienten $(n=6)$ wurde die Verknöcherung intraoperativ entfernt. Im weiteren Verlauf trat in $83 \%(n=5)$ der Fälle keine erneute Ossifikation auf, bei $17 \%$ der Kinder $(n=1)$ nach vier Verlängerungsoperationen dagegen schon. Die Wachstumsrate der Ossifikationen wies sehr variable Verläufe auf (Abb.15, Abb.16).

Abb.15:

Darstellung der Größe der Ossifikationen in $\mathrm{mm}^{2}$ während der Behandlungsdauer in Monaten:

Die Größe der Ossifikationen bei den 13 betroffenen Patienten im Verlauf der Behandlung in Monaten.

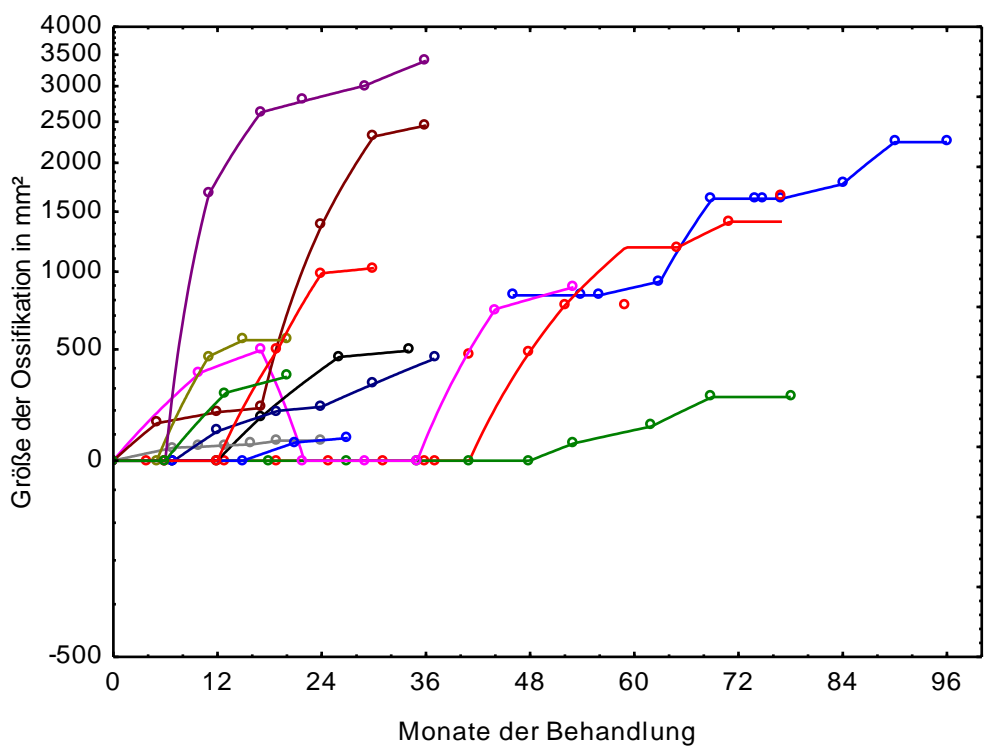

Abb.16:

Darstellung der Größe der Ossifikationen in $\mathrm{mm}^{2}$ bei unterschiedlicher Anzahl an Operationen:

Die Größe der Ossifikationen bei den 13 betroffenen Patienten im Verhältnis zur Anzahl der Operationen.

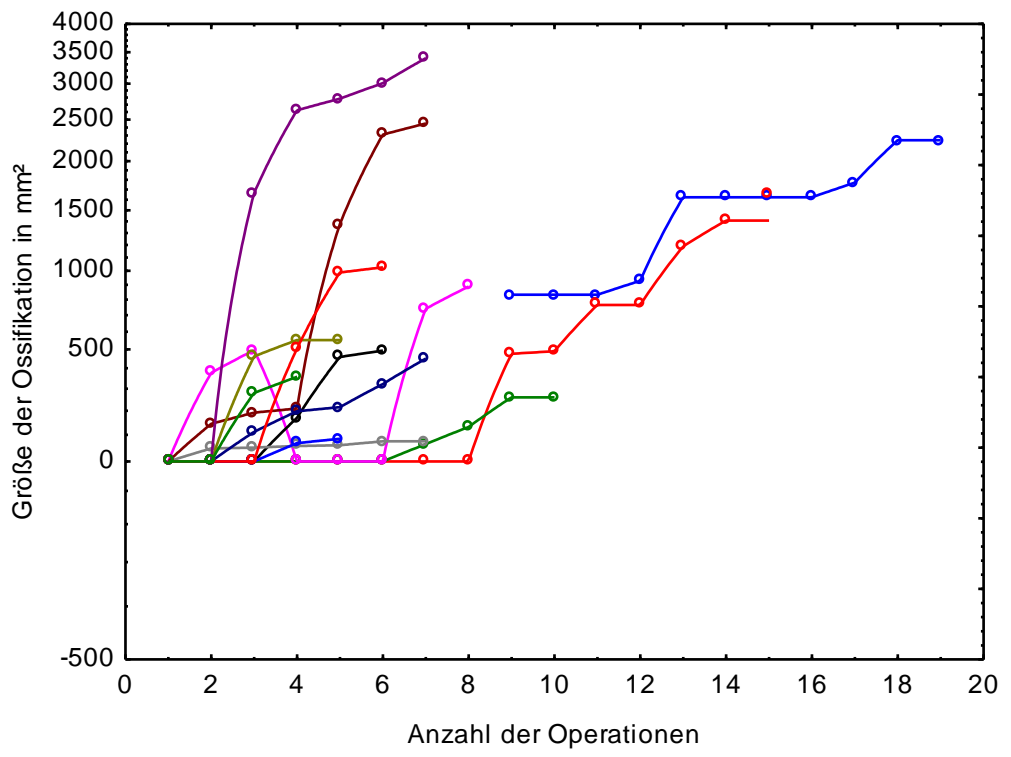

\subsubsection{Einflussgrößen auf die Ausbildung von Ossifikationen}

Anhand des Patientenkollektivs wurde untersucht, welche vermeintlichen Einflussgrößen unter Berücksichtigung der unterschiedlich langen Behandlungsdauer bzw. unterschiedlichen Anzahl an Operationen einen signifikanten Einfluss auf das Auftreten einer Ossifikation hatten (Tab.3). 


\begin{tabular}{|c|c|c|c|}
\hline Tab.3: & Einflussgröße & $\begin{array}{c}\text { signifikanten Einfluss } \\
\text { auf Ossifikation }\end{array}$ & $\begin{array}{c}\text { keinen signifikanten } \\
\text { Einfluss auf Ossifikation }\end{array}$ \\
\hline & $\begin{array}{l}\text { Geschlecht } \\
\text { Grunderkrankung } \\
\text { VEPTR-System } \\
\text { Alter bei der Primär- } \\
\text { Implantation } \\
\text { Cobb-Winkel prä Primär- } \\
\text { Implantation } \\
\text { Cobb-Winkel post } \\
\text { Primär-Implantation } \\
\text { Differenz des Cobb- } \\
\text { Winkels prä und post } \\
\text { Primär-Implantation }\end{array}$ & $X^{*}$ & $\begin{array}{l}x \\
x \\
x \\
x \\
x \\
x\end{array}$ \\
\hline
\end{tabular}

*Bei der Differenz der Cobb-Winkel prä und post Primär-Implantation bestand eine im Durchschnitt signifikante $(p=0,005)$ Differenz von $11,6^{\circ}$ zwischen den Patienten mit bzw. ohne Ossifikation (Abb.17).

Abb.17:

Darstellung der Differenz der Cobb-Winkel prä und post Primär-Implantation in Abhängigkeit von der Ossifikation:

Patienten, bei denen eine Ossifikation auftrat, hatten im Mittel eine signifikant kleinere Differenz der Cobb-Winkel prä und post Primär-Implantation als Kinder, die keine Verknöcherung aufwiesen $\left(24,7^{\circ}\right.$ zu $\left.36,3^{\circ}\right)$.

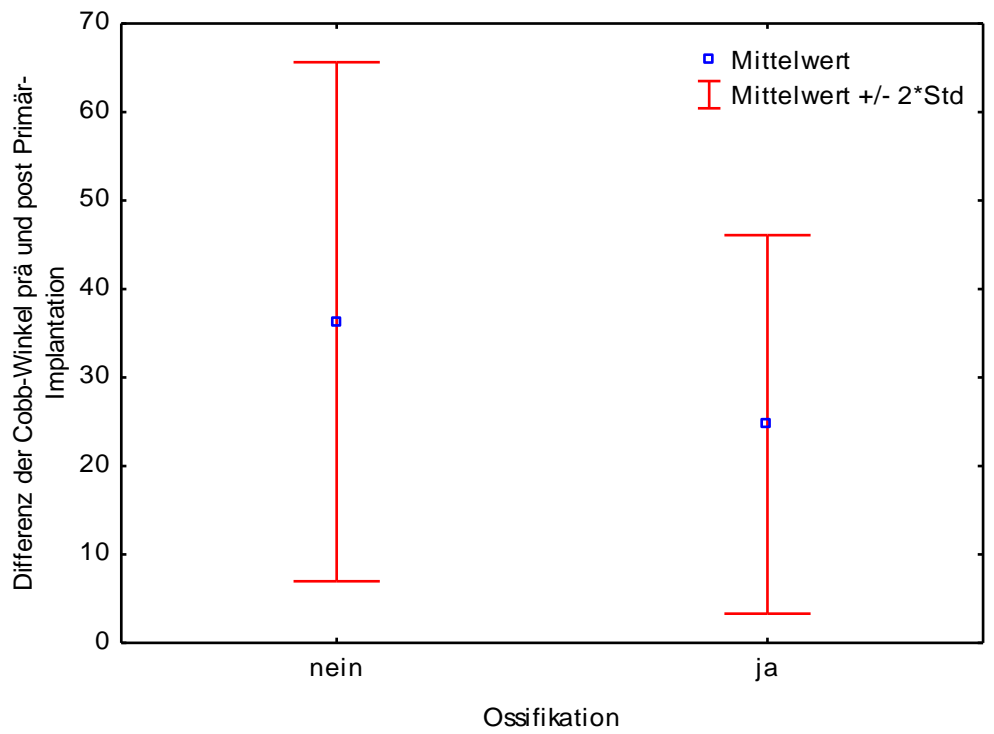

Unter Berücksichtigung der unterschiedlich langen Behandlungsdauer bzw. unterschiedlichen Anzahl an Operationen blieb diese signifikante Differenz im Mittel bestehen ( $p=0,045$ bzw. $p=0,028$ ). 


\subsubsection{Vergleich der Behandlungsdauer, der Operationsanzahl und der Komplikationen}

Anhand des Patientenkollektivs hatten Kinder, die eine Ossifikation bekamen, im Mittel eine signifikant $(p=0,002)$ längere Behandlungsdauer und signifikant $(p=0,004)$ mehr Operationen als Patienten ohne Verknöcherung. Durchschnittlich hatten diese Kinder 4,3 Operationen mehr (Abb.18) und die Behandlungsdauer war im Mittel 24,3 Monate länger (Abb.19).

Abb.18:

Darstellung der Anzahl an Operationen in Abhängigkeit von der Ossifikation:

Patienten, die eine Ossifikation bekamen, hatten im Mittel signifikant mehr Operationen (9,2 zu 4,9 Operationen).

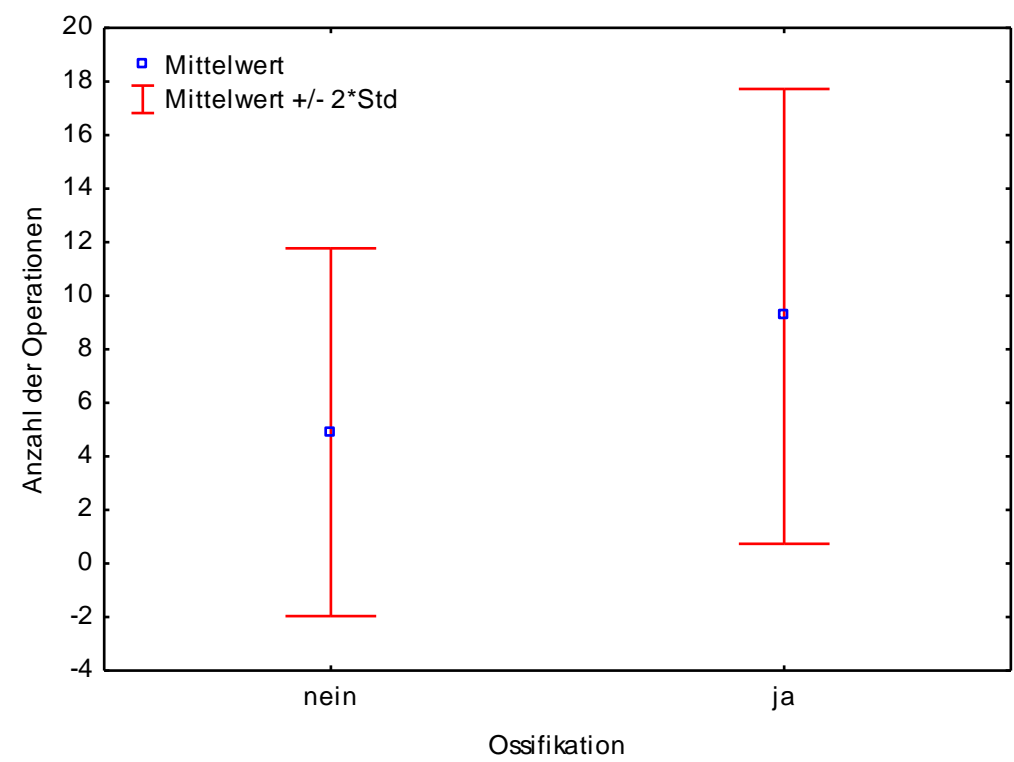

Abb.19:

Darstellung der Behandlungsdauer in Monaten in Abhängigkeit von der Ossifikation:

Bei Patienten, die eine Ossifikation bekamen, dauerte die Behandlung im Mittel signifikant länger (48,3 zu 24 Monate).

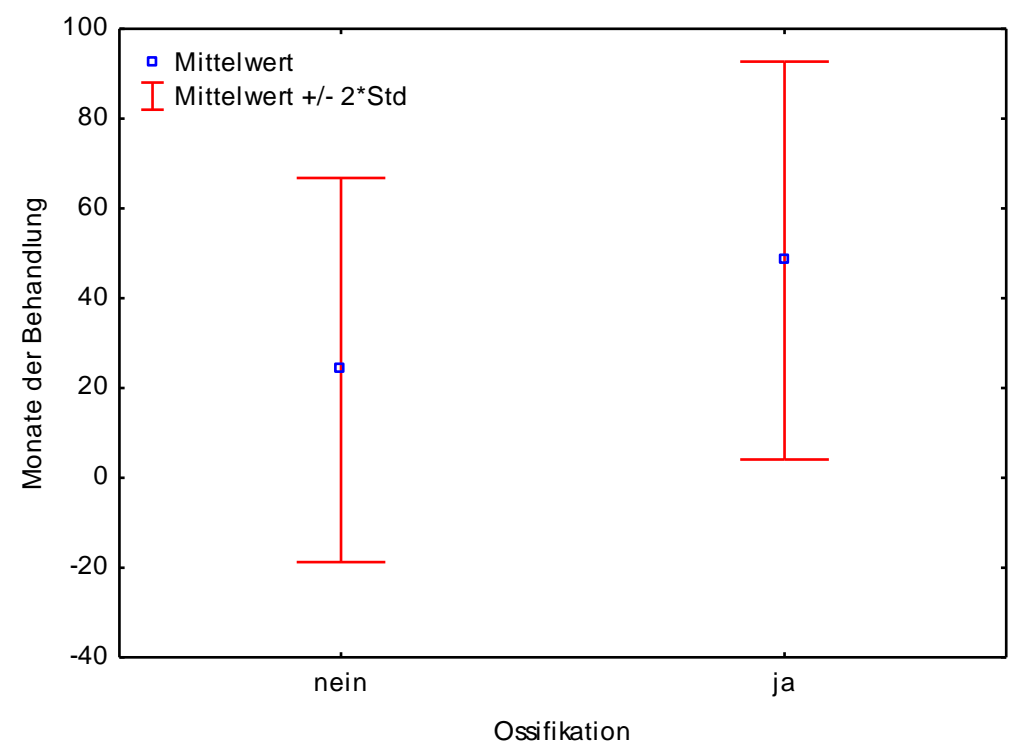

Unter Berücksichtigung der unterschiedlich langen Behandlungsdauer bzw. der unterschiedlichen Anzahl an Operationen bestand anhand des Kollektivs im Mittel kein signifikanter Unterschied im Hinblick auf die Komplikationen wie ImplantatBruch, -Infektion und -Ausriss zwischen Kindern mit bzw. ohne Ossifikation. 


\subsection{Implantat-Wanderung}

\subsubsection{Inzidenz und Lokalisation}

Anhand des Patientenkollektivs $(n=57)$ trat bei 93\% der Patienten $(n=53)$ eine Implantat-Wanderung auf. Bei $7 \%$ der Patienten $(n=4)$ wanderten keine Implantate, von denen $50 \%(n=2)$ jeweils nur primär implantiert und $50 \%(n=2)$ jeweils einmal verlängert wurden. Die einzelnen Implantate wanderten folgendermaßen:

- Implantat „Rippe zu LWS auf der konkaven Seite“ ( $n=30)$ :

- $\quad 77 \%$ an beiden Enden $(n=23)$

- $13 \%$ kranial $(n=4)$

- $3 \%$ kaudal $(n=1)$

- $7 \%$ gar nicht $(n=2)$

- Implantat „Rippe zu Rippe auf der konkaven Seite“ ( $n=23)$ :

- $\quad 43 \%$ an beiden Enden $(n=10)$

- $35 \%$ kranial $(n=8)$

- $13 \%$ kaudal $(n=3)$

- $9 \%$ gar nicht $(n=2)$

- Implantat „Rippe zu Becken auf der konkaven Seite“ $(n=32)$ :

- $81 \%$ kranial $(n=26)$

- $\quad 19 \%$ gar nicht $(n=6)$

- Implantat „Rippe zu Becken auf der konvexen Seite“ ( $n=20)$ :

- $80 \%$ kranial $(n=16)$

- $20 \%$ gar nicht $(n=4)$.

\subsubsection{Strecke im Verlauf der Behandlung}

Anhand des Patientenkollektivs wanderten im Vergleich zum Implantat „Rippe zu Rippe auf der konkaven Seite" die übrigen Implantate im Mittel unter Berücksichtigung der unterschiedlich langen Behandlungsdauer $(p=0,0005)$ bzw. der unterschiedlichen Anzahl an Operationen $(p=0,00004)$ signifikant um folgende Faktoren stärker (Tab.4). 


\begin{tabular}{l|c|c} 
Tab.4: & Behandlungsdauer & $\begin{array}{c}\text { Anzahl der } \\
\text { Operationen }\end{array}$ \\
\hline „Rippe zu Becken auf der konkaven Seite“ & 2 & 5 \\
„Rippe zu Becken auf der konvexen Seite“ & 2,5 & 6 \\
„Rippe zu LWS auf der konkaven Seite“ & 4,5 & 8
\end{tabular}

Folgend werden die Grafiken dargestellt, in denen verdeutlicht wird, dass das Implantat „Rippe zu LWS auf der konkaven Seite“ am schnellsten wanderte (Abb.20, Abb.21).

Abb.20:

Darstellung der kumulativen Strecke der Implantat-Wanderung in $\mathrm{mm}$ während der Behandlungsdauer in Monaten:

Das Implantat „Rippe zu LWS auf der konkaven Seite“ wanderte durchschnittlich im Verlauf der Behandlung schneller als die übrigen Implantate.

Abb.21:

Darstellung der kumulativen Strecke der Implantat-Wanderung in $\mathrm{mm}$ bei unterschiedlicher Anzahl an Operationen:

Das Implantat „Rippe zu LWS auf der konkaven Seite“ wanderte durchschnittlich im Verlauf - jeweils an den Operationszeitpunkten - schneller als die übrigen Implantate.
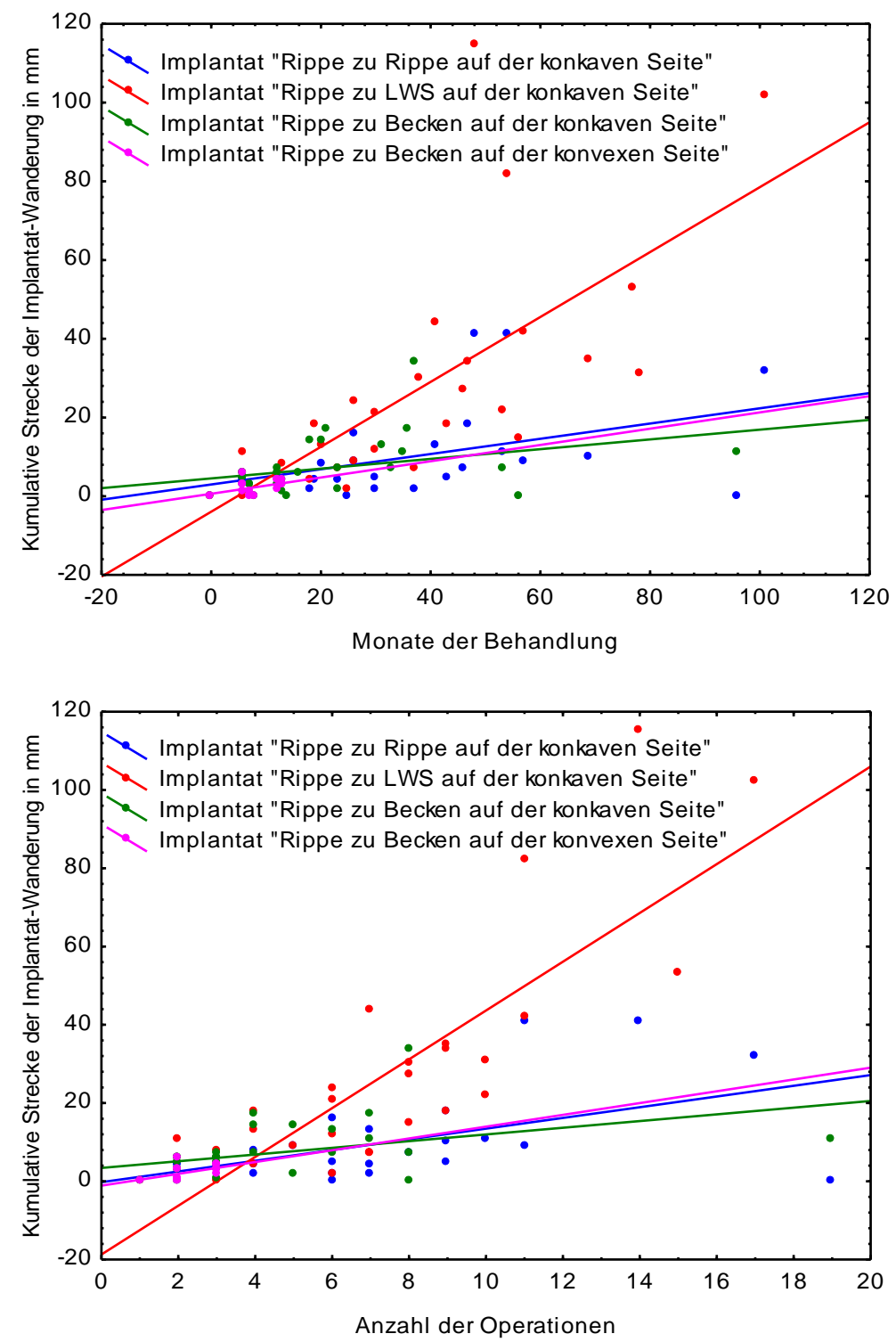
Im Patientenkollektiv konnte gezeigt werden, dass die Implantate durchschnittlich in den Operationsintervallen stärker wanderten als durch die operativen Eingriffe an sich (Tab.5).

\begin{tabular}{l|c}
\multicolumn{1}{c|}{ Implantat } & Faktor \\
\hline „Rippe zu Becken auf der konvexen Seite“ & 2 \\
„Rippe zu Becken auf der konkaven Seite“ & 2,5 \\
„Rippe zu LWS auf der konkaven Seite“ & 3,5 \\
„Rippe zu Rippe auf der konkaven Seite“ & 4
\end{tabular}

Beim Vergleich einzelner Implantate unter Berücksichtigung der unterschiedlich langen Behandlungsdauer bzw. der unterschiedlichen Anzahl an Operationen wanderten anhand des Patientenkollektivs verschiedene Implantate im Vergleich miteinander um folgende Faktoren stärker (Tab.6).

\begin{tabular}{|c|c|c|}
\hline $\begin{array}{c}\text { Tab.6: } \quad \text { Fragestellungen zum Vergleich } \\
\text { einzelner Implantate }\end{array}$ & Behandlungsdauer & $\begin{array}{l}\text { Anzahl der } \\
\text { Operationen }\end{array}$ \\
\hline $\begin{array}{l}\text { Wanderte das Implantat „Rippe zu Becken } \\
\text { auf der konkaven Seite“ stärker im System } \\
\text { "VEPTR Becken konkav einseitig“ als im } \\
\text { System „VEPTR beidseitig“? }\end{array}$ & $\begin{array}{l}\text { ja } \\
\text { signifikant } \\
(p=0,048) \\
\text { Faktor } 2\end{array}$ & $\begin{array}{c}\text { ja } \\
\text { tendenziell } \\
(p=0,127) \\
\text { Faktor } 1,75\end{array}$ \\
\hline $\begin{array}{l}\text { Wanderte das Implantat „Rippe zu LWS auf } \\
\text { der konkaven Seite“ } \underline{\text { stärker als das }} \\
\text { Implantat „Rippe zu Becken auf der } \\
\text { konkaven Seite“? }\end{array}$ & $\begin{array}{l}\text { ja } \\
\text { signifikant } \\
(\mathrm{p}=0,046) \\
\text { Faktor } 2\end{array}$ & $\begin{array}{c}\text { ja } \\
\text { tendenziell } \\
(p=0,223) \\
\text { Faktor } 1,5\end{array}$ \\
\hline $\begin{array}{l}\text { Wanderte das Implantat „Rippe zu LWS auf } \\
\text { der konkaven Seite“ stärker in einem } \\
\text { System mit einem Implantat als in einem } \\
\text { System mit zwei Implantaten? }\end{array}$ & $\begin{array}{l}\text { kein signifikanter } \\
\text { Unterschied } \\
(p=0,599)\end{array}$ & $\begin{array}{c}\text { kein signifikanter } \\
\text { Unterschied } \\
(p=0,833)\end{array}$ \\
\hline
\end{tabular}


Folgend sind die Grafiken zur Erläuterung von Tabelle sechs dargestellt (Abb.22-25).

Abb.22:

Darstellung der kumulativen Strecke der Implantat-Wanderung des Implantats „Rippe zu Becken auf der konkaven Seite“ in $\mathrm{mm}$ während der Behandlungsdauer in Monaten:

Das Implantat „Rippe zu Becken auf der konkaven Seite" wanderte im Mittel im Verlauf der Behandlung im System „VEPTR Becken konkav einseitig“ signifikant $(p=0,048)$ stärker als im System „VEPTR beidseitig“.

Abb.23:

Darstellung der kumulativen Strecke der Implantat-Wanderung des Implantats „Rippe zu Becken auf der konkaven Seite“ in $\mathrm{mm}$ bei unterschiedlicher Anzahl an Operationen:

Das Implantat „Rippe zu Becken auf der konkaven Seite" wanderte durchschnittlich im Verlauf - jeweils an den Operationszeitpunkten - im System „VEPTR Becken konkav einseitig" tendenziell $(p=0,127)$ stärker als im System „VEPTR beidseitig“.

Abb.24:

Darstellung der kumulativen Strecke der Implantat-Wanderung in mm während der Behandlungsdauer in Monaten:

Das Implantat „Rippe zu LWS auf der konkaven Seite" wanderte im Mittel im Verlauf der Behandlung signifikant $(p=0,046)$ schneller als das Implantat „Rippe zu Becken auf der konkaven Seite".
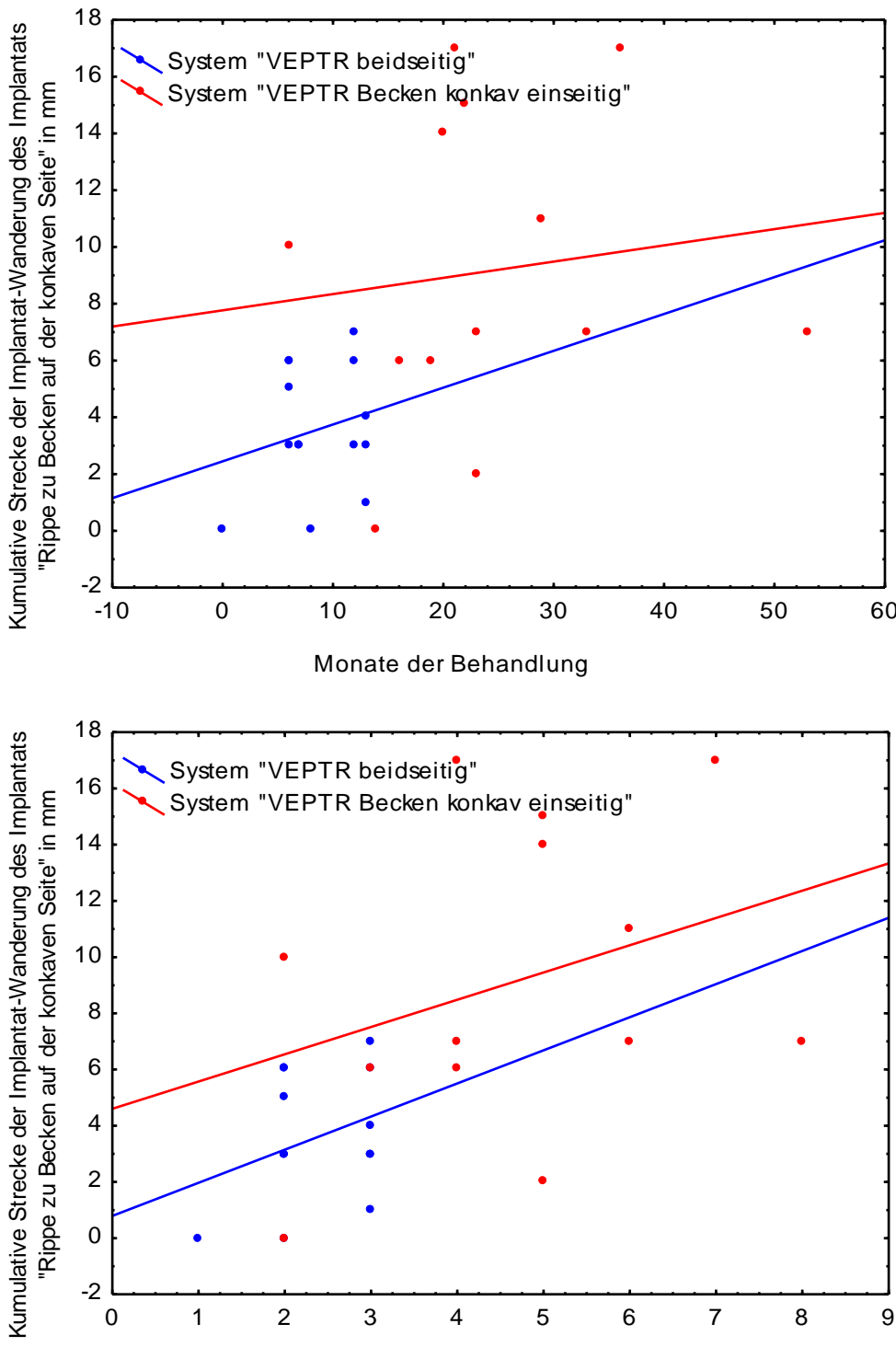

Anzahl der Operationen

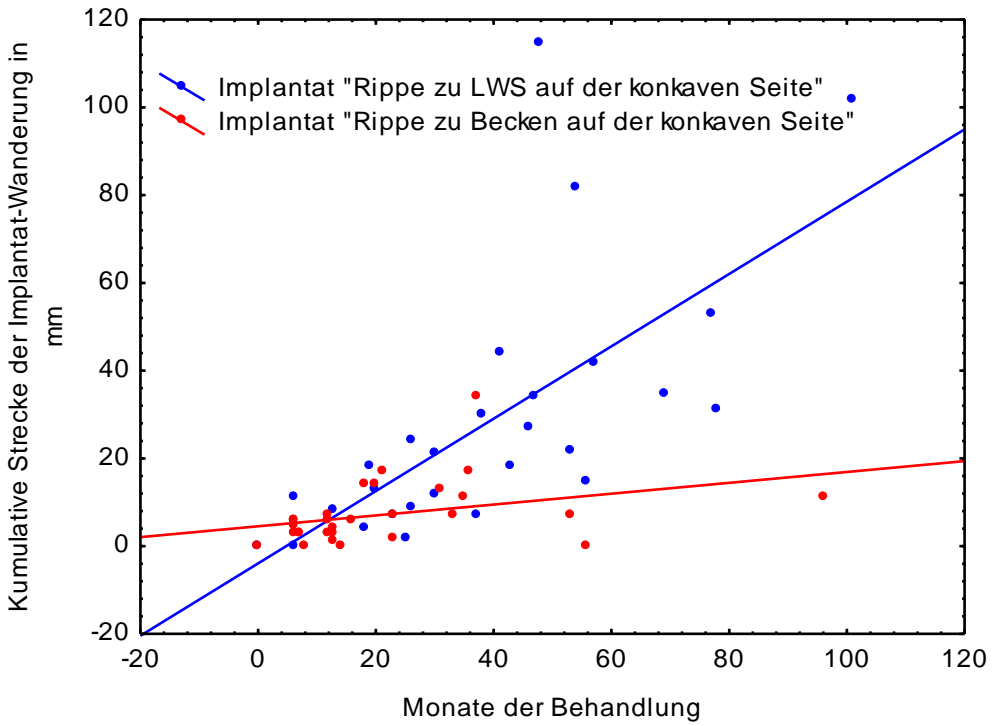


Abb.25:

Darstellung der kumulativen Strecke der Implantat-Wanderung in $\mathrm{mm}$ bei unterschiedlicher Anzahl an Operationen:

Das Implantat „Rippe zu LWS auf der konkaven Seite" wanderte durchschnittlich im Verlauf - jeweils an den Operationszeitpunkten - tendenziell $(p=0,223)$ schneller als das Implantat „Rippe zu Becken auf der konkaven Seite“.

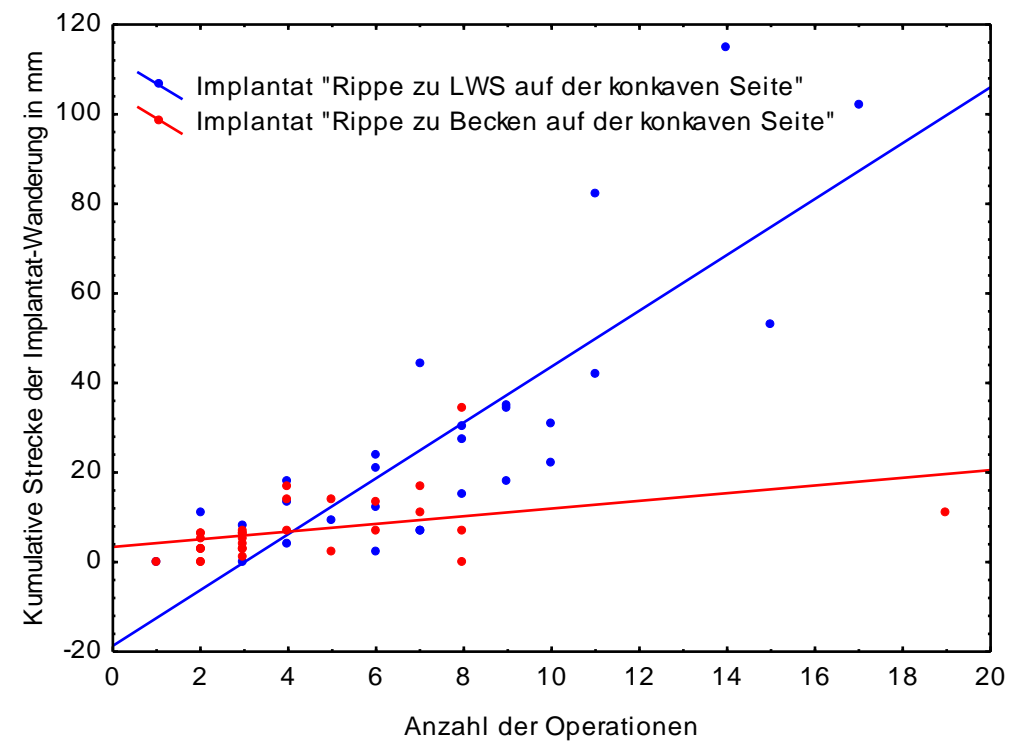

Beim Vergleich weiterer Implantate anhand des Patientenkollektivs wurde zusätzlich die Abhängigkeit der Wanderung eines Implantats von der Wanderung eines zweiten Implantats analysiert (Tab.7).

Tab.7:Fragestellungen zum Vergleich einzelner Implantate

\begin{tabular}{|c|c} 
Signifikanz & $\begin{array}{c}\text { Bestand eine Abhängigkeit } \\
\text { der Wanderung des } \\
\text { zweiten Implantats von der } \\
\text { Wanderung des ersten } \\
\text { Implantats? }\end{array}$ \\
\hline $\begin{array}{c}\text { ja } \\
(p=0,029) \\
\text { Faktor 1,5 }\end{array}$ & $\begin{array}{c}\text { ja } \\
\text { signifikant } \\
(p=0,012)\end{array}$ \\
ja & ja \\
$(p=0,0004)$ & signifikant \\
Faktor 2,75 & $(p=0,000)$
\end{tabular}

konkaven Seite“? 
Folgend sind die Grafiken zur Erläuterung von Tabelle sieben dargestellt (Abb.26, Abb.27).

Abb.26:

Darstellung der Strecke der ImplantatWanderung des Implantats „Rippe zu Becken auf der konvexen Seite" in $\mathrm{mm}$ im Verhältnis zur Strecke der ImplantatWanderung des Implantats „Rippe zu Becken auf der konkaven Seite" in mm:

Das Verhältnis zwischen der Wanderung des Implantats „Rippe zu Becken auf der konvexen Seite“ zum Implantat "Rippe zu Becken auf der konkaven Seite" ist im Mittel signifikant $(p=0,012)$ abhängig:

Wanderte das Implantat auf der konkaven Seite der Skoliose, so wanderte auch das Implantat auf der konvexen Seite.

Abb.27:

Darstellung der Strecke der ImplantatWanderung des Implantats „Rippe zu Rippe auf der konkaven Seite" in $\mathrm{mm}$ im Verhältnis zur Strecke der ImplantatWanderung des Implantats „Rippe zu LWS auf der konkaven Seite“ in mm:

Das Verhältnis zwischen der Wanderung des Implantats „Rippe zu Rippe auf der konkaven Seite“ zum Implantat „Rippe zu LWS auf der konkaven Seite“ ist im Mittel signifikant $(p=0,0004)$ abhängig:

Wanderte das Implantat „Rippe zu LWS auf der konkaven Seite", so wanderte auch das Implantat „Rippe zu Rippe auf der konkaven Seite“.
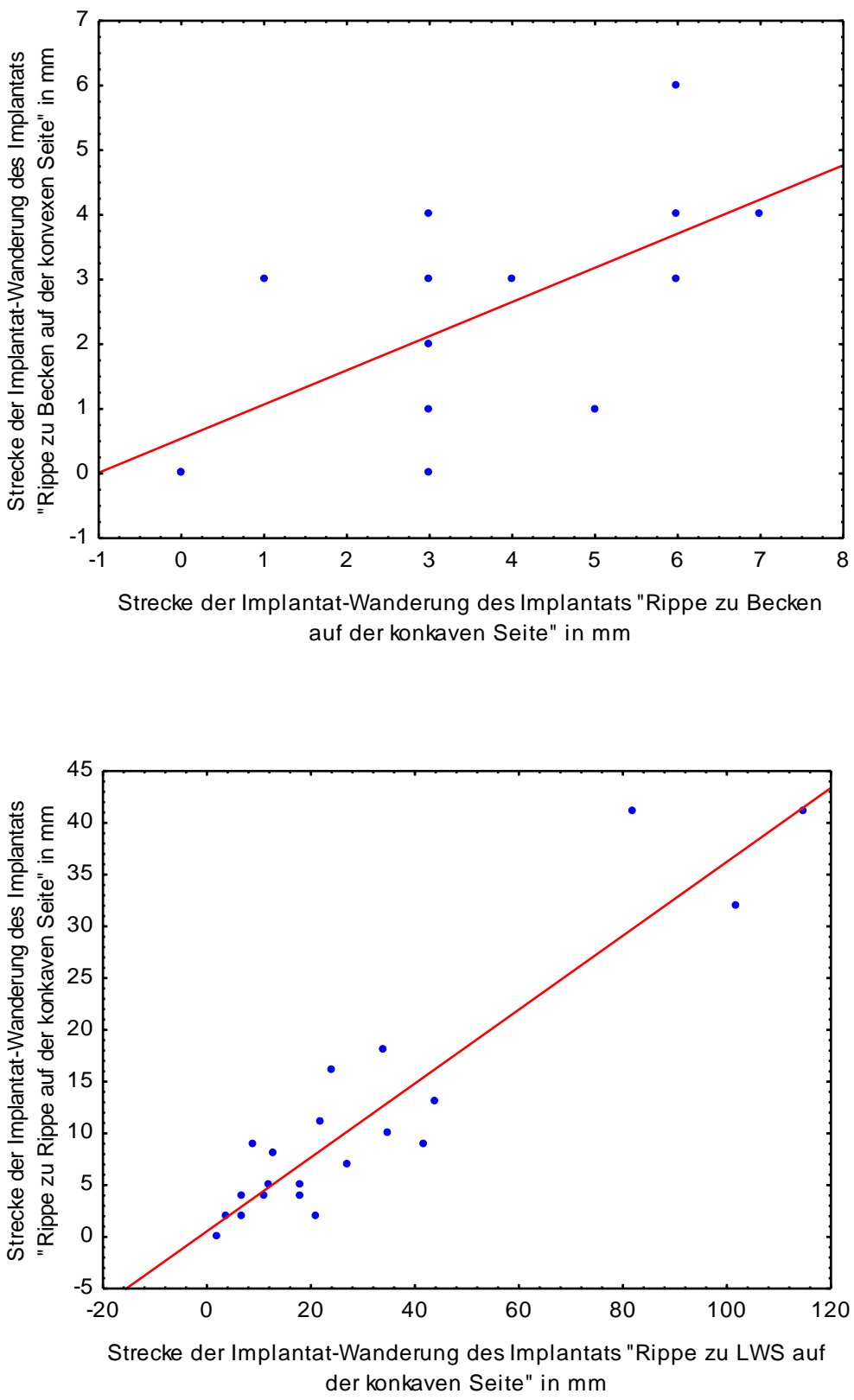

\subsubsection{Einflussgrößen auf das Entstehen der Implantat-Wanderung}

Anhand des Patientenkollektivs wurde untersucht, welche vermeintlichen Einflussgrößen einen signifikanten Einfluss auf die Implantat-Wanderung hatten (Tab.8). 


\begin{tabular}{l|c|c} 
Tab.8: & $\begin{array}{c}\text { Eignifikanten Einfluss } \\
\text { auf Implantat- } \\
\text { Wanderung }\end{array}$ & $\begin{array}{c}\text { keinen signifikanten } \\
\text { Einfluss auf Implantat- } \\
\text { Wanderung }\end{array}$ \\
\hline - Geschlecht & & $\mathrm{X}$ \\
- Grunderkrankung & & $\mathrm{X}$ \\
- VEPTR-System & \\
- Alter bei der Primär- & & \\
- Implantation & & \\
Cobb-Winkel prä & $\mathrm{X}^{*}$ & $\mathrm{X}$ \\
Primär-Implantation & & $\mathrm{X}$ \\
Cobb-Winkel post & & \\
Primär-Implantation & & \\
Differenz des Cobb- \\
Winkels prä und post \\
Primär-Implantation
\end{tabular}

*Unter Berücksichtigung der unterschiedlich langen Behandlungsdauer bzw. der unterschiedlichen Anzahl an Operationen hatte die Größe des Cobb-Winkels prä Primär-Implantation einen signifikanten Einfluss ( $p=0,029$ bzw. $p=0,044)$ in Bezug auf die Wanderung des Implantats „Rippe zu Becken auf der konkaven Seite“. Bei den $50 \%$ der Patienten mit dem größeren Cobb-Winkel prä Primär-Implantation wanderte dieses Implantat im Mittel etwa um den Faktor zwei stärker als bei den $50 \%$ der Kinder mit dem kleineren Winkel.

\subsection{Sonstige Komplikationen}

\subsubsection{Implantat-Infektion}

Anhand des Patientenkollektivs musste bei $9 \%$ der Patienten $(n=5)$ ein Implantat aufgrund einer Infektion vorzeitig explantiert werden. In der Folge wurde diese Komplikation auf unterschiedliche Weise gehandhabt: 
- $40 \%(n=2)$ wurden durch ein neues Implantat ersetzt

- $20 \%(n=1)$ wurden nicht durch ein neues Implantat ersetzt

- $40 \%(n=2)$ konnten aufgrund des Studienendes nicht beurteilt werden.

\subsubsection{Implantat-Ausriss}

In dem untersuchten Kollektiv $(n=57)$ lag bei 19\% der Patienten $(n=11)$ ein ImplantatAusriss vor (Abb.28).

Abb.28:

Implantat-Ausriss am kaudalen Ende des Implantats

"Rippe zu LWS auf der konkaven Seite" (rotes

Rechteck) bei Patientin P. A. M.. Die kaudale

Verankerung sollte normalerweise am Pedikel eines

Wirbelkörpers befestigt sein.

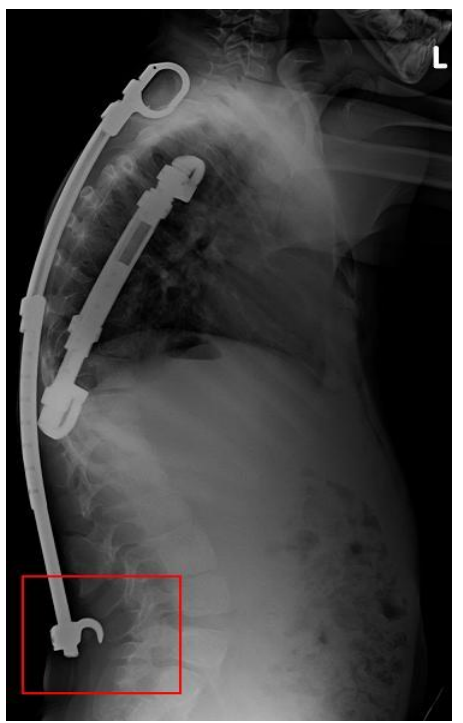

Die Implantate $(n=11)$ rissen an folgenden Strukturen aus:

- $82 \%$ : Rippen $(\mathrm{n}=9)$

- $18 \%$ : Lendenwirbelsäule $(n=2)$.

Bei der Analyse der einzelnen Implantate war die Verteilung der Implantat-Ausrisse folgendermaßen:

- $23 \%(n=7)$ der Implantate „Rippe zu LWS auf der konkaven Seite“ $(n=30)$

- $71 \%$ kranial $(n=5)$

- $29 \%$ kaudal $(n=2)$

- $13 \%(n=4)$ der Implantate „Rippe zu Becken auf der konkaven Seite“ ( $n=32)$

- $100 \%$ kranial $(n=4)$

- $0 \%(n=0)$ der Implantate „Rippe zu Rippe auf der konkaven Seite“ $(n=23)$

- $0 \%(n=0)$ der Implantate „Rippe zu Becken auf der konvexen Seite“ $(n=20)$. 
Anhand des Patientenkollektivs wurden Patienten, bei denen ein Implantat ausriss, tendenziell $(p=0,091)$ im Durchschnitt häufiger operiert, als Kinder, die keinen Implantat-Ausriss hatten. Das Mittel der Operationsanzahl lag bei acht zu 5,5 Operationen.

Des Weiteren wurde analysiert, dass Patienten, bei denen ein Implantat ausriss, tendenziell $(p=0,063)$ im Mittel einen größeren Cobb-Winkel prä Primär-Implantation $\left(92,2^{\circ}\right)$ hatten, als Kinder ohne Implantat-Ausriss $\left(74,5^{\circ}\right)$. Unter Berücksichtigung der unterschiedlichen Anzahl an Operationen blieb diese Tendenz $(p=0,068)$ bestehen; unter Berücksichtigung der unterschiedlich langen Behandlungsdauer zeigte sich eine Signifikanz $(p=0,041)$.

Ferner zeigte sich, dass Patienten, bei denen ein Implantat ausriss, im Mittel einen signifikant $(p=0,034)$ größeren Cobb-Winkel post Primär-Implantation $\left(62,1^{\circ}\right)$ hatten, als Kinder ohne Implantat-Ausriss (39, Abb.29). Unter Berücksichtigung der unterschiedlich langen Behandlungsdauer bzw. der unterschiedlichen Anzahl an Operationen blieb diese Signifikanz ( $p=0,014$ bzw. $p=0,021)$ bestehen.

Abb.29:

Darstellung des Cobb-Winkels post Primär-Implantation in Abhängigkeit vom Implantat-Ausriss:

Der Cobb-Winkel post Primär-Implantation war bei Patienten, die einen ImplantatAusriss hatten, im Mittel signifikant $(p=0,034)$ größer. Die Differenz betrug durchschnittlich $23,1^{\circ}$.

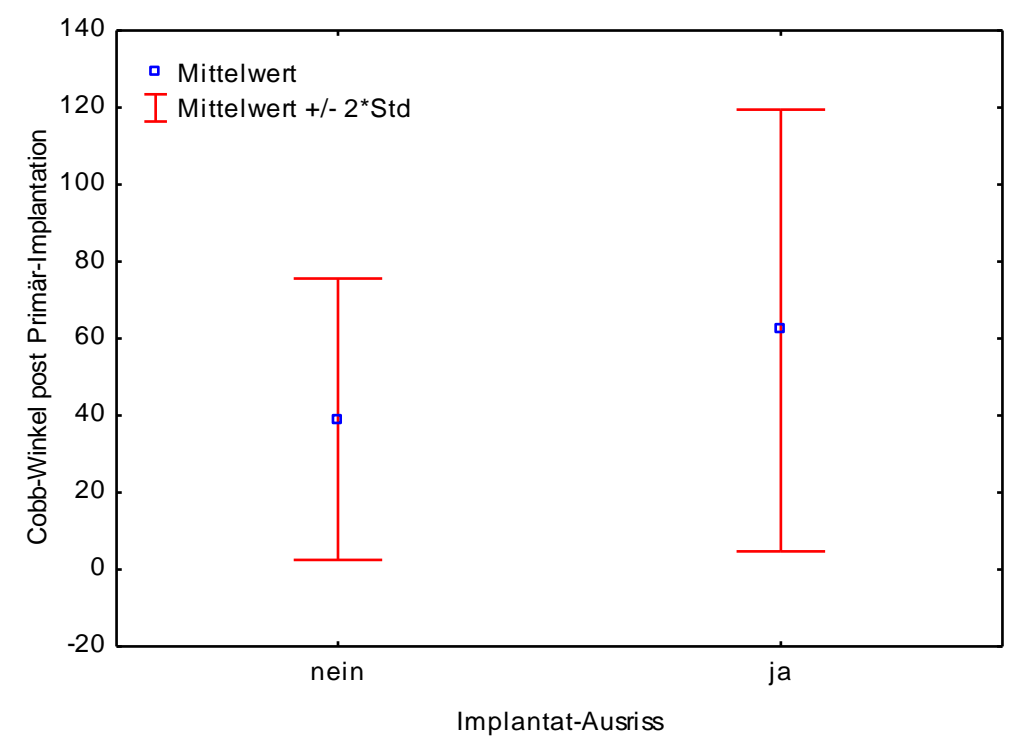

\subsubsection{Implantat-Bruch}

In unserem Patientenkollektiv $(n=57)$ trat bei $7 \%$ der Patienten $(n=4)$ ein ImplantatBruch auf (Abb.30). Bei 25\% $(n=1)$ dieser Kinder $(n=4)$ brach zweimal ein Implantat. Die Implantate waren folgendermaßen betroffen: 
- $60 \%$ : Implantate „Rippe zu Becken auf der konkaven Seite“ $(n=3)$

- $40 \%$ : Implantate „Rippe zu LWS auf der konkaven Seite“ (n=2).

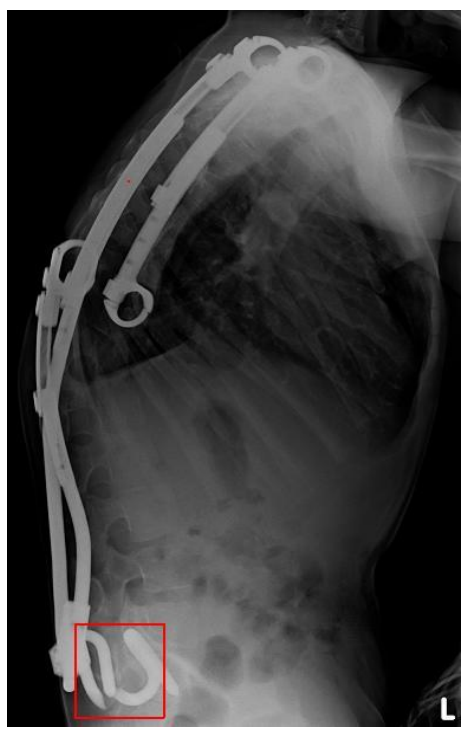

\subsubsection{Rippen-Spreizung nach Primär-Implantation}

Anhand des auszuwertenden Kollektivs wurde nach der primären Implantation bei 91\% der Patienten $(n=51)$ die jeweilige Rippe, an der das kraniale Ende des Implantats verankert worden war, nach oben gespreizt (Abb.31). Bei den betroffenen Kindern $(n=51)$ trat dies zu 86\% $(n=44)$ jeweils einmal und zu 14\% $(n=7)$ jeweils aufgrund eines später zusätzlich verwendeten Implantats zweimal auf.

Abb.31:

Rippen-Spreizung nach Primär-Implantation (rechtes Bild, rote Pfeile) im Vergleich zum Zeitpunkt vor der Implantation (linkes Bild, rote Pfeile) bei Patient J. A..
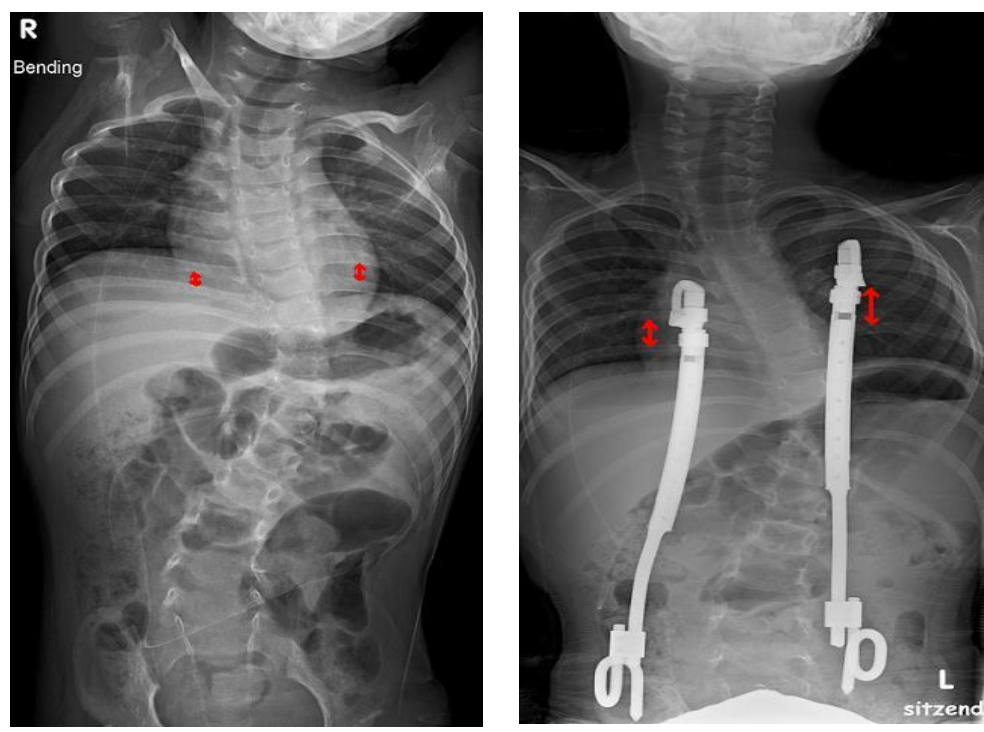
Des Weiteren bestand unter Berücksichtigung der unterschiedlich langen Behandlungsdauer bzw. der unterschiedlichen Anzahl an Operationen eine Tendenz, dass Patienten mit einer Rippen-Spreizung im Mittel sowohl einen größeren CobbWinkel prä Primär-Implantation ( $p=0,07$ bzw. $p=0,064)$ als auch eine größere Differenz der Cobb-Winkel prä und post Primär-Implantation ( $p=0,093$ bzw. $p=0,074)$ hatten, als Kinder ohne Rippen-Spreizung.

\subsection{Operativer Verlauf während der Nachuntersuchung}

\subsubsection{VEPTR-System}

\section{Nachträglicher Implantat-Einbau}

Im Nachuntersuchungszeitraum wurde in unserem Patientenkollektiv $(n=57)$ elfmal nachträglich ein Implantat verwendet. Dies betraf $18 \%$ der Patienten $(n=10)$, wobei 10\% $(n=1)$ dieser Kinder zwei Implantate zusätzlich implantiert bekamen. Der Grund hierfür ist die sich in manchen Fällen sehr stark ändernde Statik beim wachsenden bzw. über Jahre mit dem VEPTR behandelten Kind. Die Implantate wurden unterschiedlich oft nachträglich implantiert:

- 55\%: Implantate „Rippe zu Becken auf der konvexen Seite“ ( $n=6)$

- $27 \%$ : Implantate „Rippe zu Becken auf der konkaven Seite“ $(n=3)$

- 9\%: Implantate „Rippe zu LWS auf der konkaven Seite“ $(n=1)$

- 9\%: Implantate „Rippe zu Rippe auf der konkaven Seite“ ( $n=1)$.

Unter Berücksichtigung der unterschiedlich langen Behandlungsdauer bzw. der unterschiedlichen Anzahl an Operationen bestand anhand des Patientenkollektivs im Mittel eine Signifikanz ( $p=0,032$ bzw. $p=0,017)$ dafür, dass im System „VEPTR Becken konkav einseitig“ häufiger ein Implantat nachträglich implantiert wurde.

\section{Umbau des VEPTR-Systems}

In unserem Patientenkollektiv wurde das VEPTR-System während des operativen Verlaufs bei $9 \%$ der Patienten $(n=5)$ umgebaut. Es wurde viermal vom System „VEPTR Becken konkav einseitig“ und einmal vom System „VEPTR konkav einseitig“ auf das System „VEPTR Becken beidseitig“ umgestiegen. Der Grund hierfür ist 
ebenfalls die sich in manchen Fällen stark ändernde Statik beim wachsenden bzw. über Jahre mit dem VEPTR behandelten Kind.

\section{Implantat-Ausbau}

In unserem Patientenkollektiv wurden bei 16\% der Patienten $(n=9) 16$ Implantate explantiert. Der Grund für diese Explantationen waren in $56 \%$ der Fälle $(n=5)$ eine Implantat-Infektion und in $44 \%$ der Fälle $(n=4)$ eine die VEPTR-Behandlung beendende Spondylodese. Die Implantate wurden unterschiedlich oft explantiert:

- 31\%: Implantate „Rippe zu LWS auf der konkaven Seite“ ( $n=5)$

- 31\%: Implantate „Rippe zu Rippe auf der konkaven Seite“ ( $n=5)$

- $19 \%$ : Implantate „Rippe zu Becken auf der konkaven Seite“ ( $n=3)$

- 19\%: Implantate „Rippe zu Becken auf der konvexen Seite“ $(n=3)$.

\subsubsection{Einzelne Implantate}

Implantat-Komponenten-Austausch

In unserem Patientenkollektiv wurde 28mal eine Komponente eines Implantats ausgetauscht. Der Grund hierfür lag darin, dass das Implantat aufgrund des Wachstums des Kindes zu klein geworden war. Dieser Komponenten-Austausch betraf $32 \%$ der Patienten $(n=18)$ und unterschiedlich oft die verschiedenen Implantate:

- 50\%: Implantate „Rippe zu Rippe auf der konkaven Seite“ ( $n=14)$

- $32 \%$ : Implantate „Rippe zu LWS auf der konkaven Seite“ ( $\mathrm{n}=9)$

- $14 \%$ : Implantate „Rippe zu Becken auf der konkaven Seite“ $(n=4)$

- 4\%: Implantate „Rippe zu Becken auf der konvexen Seite“ $(n=1)$.

Die beiden ersten Implantate waren prozentual öfter betroffen (61\% bzw. 29\%) als die beiden anderen (13\% bzw. 5\%). In unserem Kollektiv hatten Kinder, bei denen eine Komponente des Implantats gewechselt wurde, durchschnittlich eine signifikant längere Behandlungsdauer $(p=0,000002)$ und signifikant mehr Operationen $(p=0,000006)$, als Patienten, bei denen keine Komponente ausgetauscht wurde. Das Verhältnis war im Mittel 53,6 zu 18,8 Monate bzw. 9,8 zu 4,2 Operationen. 


\section{Implantat-Umsetzung}

In unserem Patientenkollektiv wurde 25mal ein Implantat umgesetzt. Der Grund war in 96\% der Fälle ein Statikproblem des VEPTR-Systems $(n=24)$ und in 4\% der Fälle eine Ossifikation $(n=1)$. Die Probleme der Statik $(n=24)$ waren zu 37,5\% durch einen Implantat-Ausriss $(\mathrm{n}=9)$ und $\mathrm{zu}$ 16,5\% durch einen Implantat-Bruch $(\mathrm{n}=4)$ sicher $\mathrm{zu}$ verifizieren. In 46\% der Fälle $(n=11)$ konnte der Grund für dieses Problem anhand der Röntgenaufnahmen nicht analysiert werden. Die Implantat-Umsetzung betraf $35 \%$ der Patienten $(n=20)$. Von diesen Kindern wurde bei 75\% $(n=15)$ jeweils ein Implantat und bei 25\% $(n=5)$ jeweils zwei Implantate umgesetzt. Die Implantate waren unterschiedlich oft betroffen:

- 64\%: Implantate „Rippe zu LWS auf der konkaven Seite“ ( $n=16)$

- $32 \%$ : Implantate „Rippe zu Becken auf der konkaven Seite“ ( $n=8)$

- 4\%: Implantate „Rippe zu Becken auf der konvexen Seite“ $(n=1)$

- $0 \%$ : Implantate „Rippe zu Rippe auf der konkaven Seite“ $(n=0)$.

Die Verwendung der beiden erstgenannten jeweiligen Hauptimplantate der VEPTRSysteme führte prozentual häufiger (52\% bzw. 25\%) zu einer Umsetzung als die Implantation der beiden letztgenannten akzessorischen Implantate (5\% bzw. 0\%). In unserem Kollektiv hatten Patienten, bei denen ein Implantat umgesetzt wurde, im Mittel eine signifikant längere Behandlungsdauer $(p=0,00005)$ und signifikant mehr Operationen $(p=0,00003)$, als Kinder, bei denen kein Implantat versetzt wurde. Das Verhältnis war im Mittel 48,3 zu 19,8 Monate bzw. 9,3 zu 4,1 Operationen.

Des Weiteren bestand unter Berücksichtigung der unterschiedlich langen Behandlungsdauer eine Tendenz $(p=0,07)$, dass bei Patienten mit kongenitaler Skoliose im Mittel häufiger (56\%) ein Implantat umgesetzt wurde, als bei Kindern mit anderen Grunderkrankungen (SMA 0\%, MMC 20\%, Sonstige 39\%).

Zudem hatten Patienten, bei denen ein Implantat umgesetzt wurde, unter Berücksichtigung der unterschiedlich langen Behandlungsdauer im Mittel einen signifikant $(p=0,025)$ größeren Cobb-Winkel prä Primär-Implantation, als Kinder, bei denen kein Implantat versetzt wurde. Eine Tendenz $(p=0,064)$ bestand unter Berücksichtigung der unterschiedlichen Anzahl an Operationen.

Ferner hatten Patienten, bei denen ein Implantat umgesetzt wurde, unter Berücksichtigung der unterschiedlich langen Behandlungsdauer bzw. der 
unterschiedlichen Anzahl an Operationen im Mittel einen signifikant ( $p=0,014$ bzw. $p=0,009)$ größeren Cobb-Winkel post Primär-Implantation, als Kinder, bei denen kein Implantat versetzt wurde.

Abb.32:

Darstellung des Cobb-Winkels post Primär-Implantation in Abhängigkeit von der Implantat-Umsetzung:

Der Cobb-Winkel post Primär-Implantation war bei Patienten, die eine ImplantatUmsetzung hatten, im Mittel signifikant $(p=0,002)$ größer. Die Differenz betrug durchschnittlich $21,3^{\circ}$.

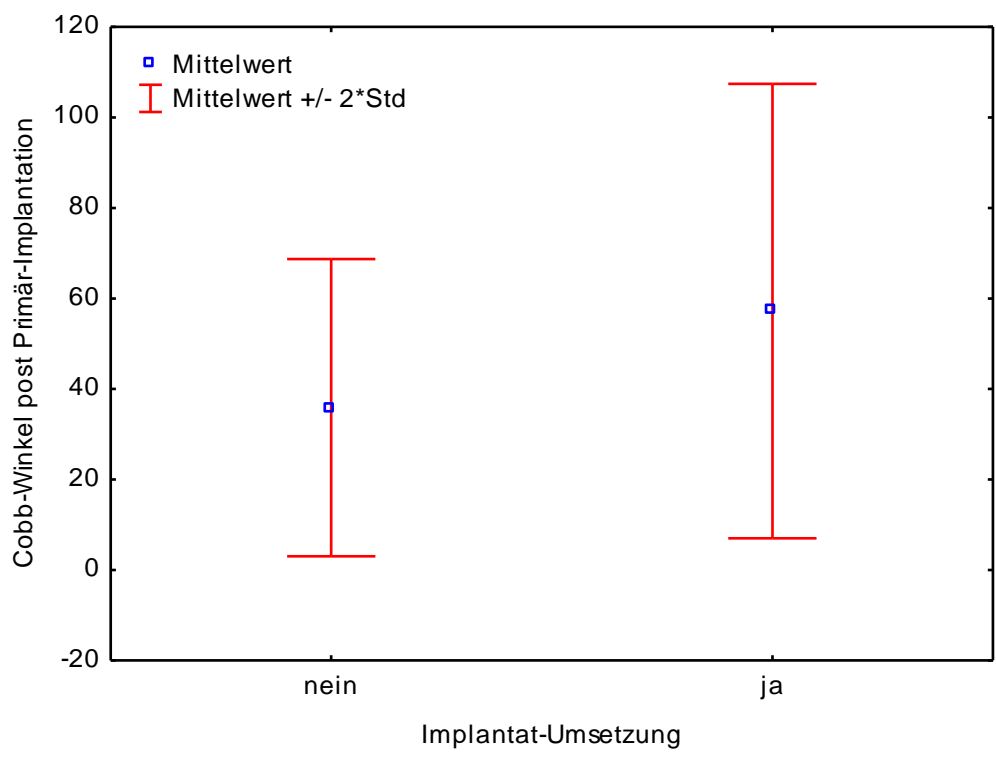

\subsubsection{Cobb- und Kyphose-Winkel}

\section{Cobb-Winkel}

In unserem Patientenkollektiv $(n=57)$ hatten 98\% der Kinder $(n=56)$ eine einbogige Skoliose (Abb.33) und $2 \%$ der Patienten $(n=1)$ eine doppelbogige Skoliose.

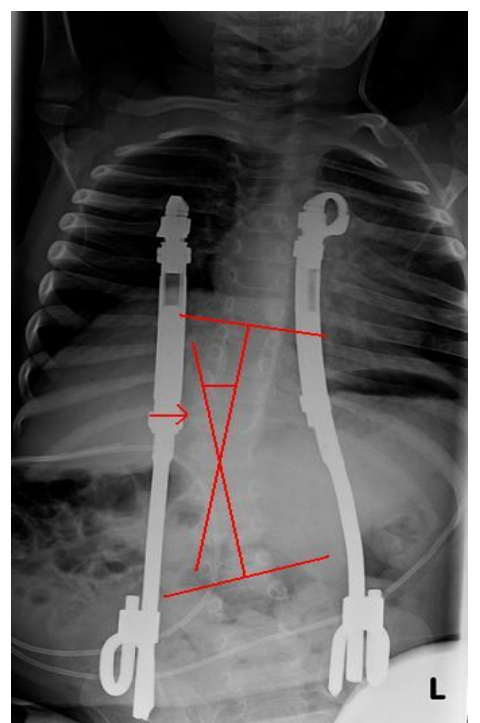

In dem auszuwertenden Kollektiv $(n=55)$ mit einbogiger Skoliose veränderte sich der Cobb-Winkel im Verlauf der Behandlung (Tab.9). 


\begin{tabular}{l|c|c} 
Tab.9: & $\begin{array}{c}\text { Größe des Cobb- } \\
\text { Winkels in Grad }\end{array}$ & $\begin{array}{c}\text { Veränderung des Cobb-Winkels in } \\
\text { Prozent }\end{array}$ \\
\hline $\begin{array}{l}\text { Cobb-Winkel prä } \\
\text { Primär-Implantation }\end{array}$ & 77,7 (30 bis 140) & \\
\hline $\begin{array}{l}\text { Cobb-Winkel post } \\
\text { Primär-Implantation }\end{array}$ & 43,3 (11 bis 120) & 45\% Abnahme (0 bis 82)* \\
\hline $\begin{array}{l}\text { Cobb-Winkel nach } \\
\text { im Mittel 4,7 (0 bis } \\
\text { 17) Verlängerungs- } \\
\text { operationen }\end{array}$ & 45,5 (0 bis 97) & $\begin{array}{c}\text { Abnahme) zu prä Primär-Implantation } \\
5 \% \text { Zunahme (53\% Abnahme bis } 120 \% \\
\text { Zunahme) zu post Primär-Implantation }\end{array}$
\end{tabular}

*Die durchschnittliche Abnahme des Cobb-Winkels prä zu post Primär-Implantation war signifikant $(p=0,000)$.

Bei den 2\% der Patienten $(n=1)$ mit doppelbogiger Skoliose nahm der Cobb-Winkel von prä zu post Primär-Implantation thorakal von $104^{\circ}$ auf $68^{\circ}$ und lumbal von $62^{\circ}$ auf $50^{\circ}$ ab. Der letztmalig gemessene Cobb-Winkel nach sieben Verlängerungsoperationen betrug bei diesem Kind thorakal $64^{\circ}$ und lumbal $57^{\circ}$.

Kyphose-Winkel

In unserem Patientenkollektiv wiesen $7 \%$ der Patienten $(n=4)$ neben der Skoliose eine Hyperkyphose auf (Abb.34).

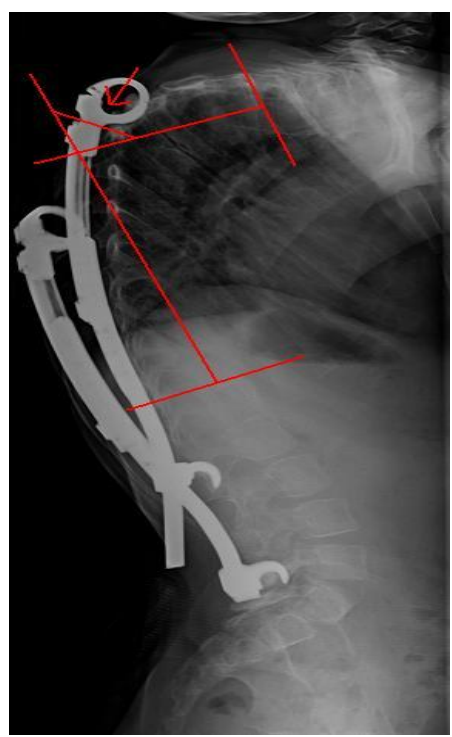


In dem auszuwertenden Kollektiv $(n=4)$ veränderte sich der Kyphose-Winkel im Verlauf der Behandlung (Tab.10).

\begin{tabular}{|c|c|c|}
\hline Tab.10: & $\begin{array}{c}\text { Größe des Kyphose- } \\
\text { Winkels in Grad }\end{array}$ & $\begin{array}{l}\text { Veränderung des Kyphose-Winkels } \\
\text { in Prozent }\end{array}$ \\
\hline $\begin{array}{l}\text { Kyphose-Winkel prä } \\
\text { Primär-Implantation }\end{array}$ & $106,5$ (82 bis 120$)$ & \\
\hline $\begin{array}{l}\text { Kyphose-Winkel post } \\
\text { Primär-Implantation }\end{array}$ & $71,5$ (50 bis 90$)$ & $32 \%$ Abnahme (17 bis 55$)^{*}$ \\
\hline $\begin{array}{l}\text { Kyphose-Winkel } \\
\text { nach im Mittel 7,8 } \\
\text { (5 bis } 12 \text { ) } \\
\text { Verlängerungs- } \\
\text { operationen }\end{array}$ & $91,5$ (81 bis 99$)$ & $\begin{array}{l}\text { 14\% Abnahme (21\% Zunahme bis } \\
29 \% \text { Abnahme) zu prä Primär- } \\
\text { Implantation } \\
20 \% \text { Zunahme ( } 3 \% \text { bis } 86 \% \text { ) zu post } \\
\text { Primär-Implantation }\end{array}$ \\
\hline
\end{tabular}

*Die durchschnittliche Abnahme des Kyphose-Winkels prä zu post PrimärImplantation war signifikant $(p=0,035)$.

\subsection{Zusammenfassung der wichtigsten Ergebnisse}

\subsubsection{Ossifikation}

Bei 24\% der Patienten traten Ossifikationen auf, die in 92\% der Fälle die Hauptimplantate der VEPTR-Systeme involvierten. Die Lendenwirbelsäule war zu 54\%, die Rippen und der Beckenkamm jeweils zu 23\% betroffen. Bei 11\% der Kinder trat eine Ossifikation in den ersten zwölf Monaten und bei 19\% in den ersten 24 Monaten auf. Die Rate an Ossifikationen nahm im Verlauf der Behandlung nicht zu. Nach 21 Monaten Behandlungsdauer waren 74\% der Patienten nicht ossifiziert und nach 53 Monaten blieben 52\% der Kinder ohne Verknöcherung. Die Größe und das Wachstum wiesen eine große Variabilität auf. Vermeintliche Einflussgrößen (Grunderkrankung, VEPTR-System, etc.) hatten im Mittel keinen signifikanten Einfluss auf das Auftreten einer Ossifikation. Bei Patienten mit einer Ossifikation zeigte sich durchschnittlich eine signifikant kleinere $\left(24,7^{\circ} \mathrm{zu} 36,3^{\circ}\right)$ Differenz der 
Cobb-Winkel prä und post Primär-Implantation. Ob ein Patient mit Ossifikation einen vermehrten Operationsaufwand bzw. vermehrte Komplikationen wie Implantat-Bruch, -Infektion und -Ausriss hatte, konnte in der vorliegenden Analyse aufgrund der geringen Fallzahl nicht abschließend geklärt werden.

\subsubsection{Implantat-Wanderung}

Mit Ausnahme von Patienten mit lediglich einer Primär-Implantation $(n=2)$ oder nur einer einmaligen Verlängerung $(n=2)$ wiesen alle übrigen Probanden $(n=53)$ eine Implantat-Wanderung auf, welche bei allen Implantatvarianten stattfand und bei $19 \%$ der Kinder zu einem Implantat-Ausriss führte. Das Implantat „Rippe zu Rippe auf der konkaven Seite“ wanderte am zweithäufigsten (91\%), dafür aber die geringste Strecke. Am häufigsten (93\%) und am stärksten der unterschiedlich langen Behandlungsdauer unter Berücksichtigung $(p=0,0005)$ bzw. der unterschiedlichen Anzahl an Operationen ( $p=0,00004)$ wanderte im Mittel das Implantat „Rippe zu LWS auf der konkaven Seite“ (Faktor 4,5 bzw. 8 im Vergleich zum Implantat „Rippe zu Rippe auf der konkaven Seite“), gefolgt vom Implantat „Rippe zu Becken auf der konvexen Seite“ (2,5 bzw. 6) und dem Implantat „Rippe zu Becken auf der konkaven Seite“ (2 bzw. 5). Die Hauptimplantate wanderten im jeweiligen VEPTR-System signifikant stärker als die jeweiligen akzessorischen Implantate. Wenn eine Implantat-Wanderung stattfand, so war immer das gesamte VEPTR-Konstrukt in Bewegung.

Vermeintliche Einflussgrößen (Grunderkrankung, Alter bei der Primär-Implantation, etc.) auf die Implantat-Wanderung waren bis auf eine Ausnahme nicht signifikant. Das Implantat „Rippe zu Becken auf der konkaven Seite“ wanderte im Mittel unter Berücksichtigung der unterschiedlich langen Behandlungsdauer bzw. der unterschiedlichen Anzahl an Operationen um den Faktor zwei signifikant $(p=0,029$ bzw. $p=0,044)$ stärker bei den $50 \%$ der Patienten, die den größeren Winkel prä Primär-Implantation hatten. 


\section{Diskussion}

\subsection{Aspekte der Behandlung von Skoliosen im Kindesalter}

Das Problem der kindlichen Skoliose besteht darin, dass durch das noch vorhandene starke Wachstumspotential im Allgemeinen die Deformität zunimmt. Daher versucht man relativ frühzeitig, die Kurvenprogression aufzuhalten. Hierzu stehen konservative und operative Verfahren zur Verfügung. Die konservative Therapie führt in der Regel bei progressiven Skoliosen im Kindesalter ohne strukturelle Fehlbildungen an der Wirbelsäule zu einer positiven Beeinflussung des Verlaufs, aber in vielen Fällen nicht zur Vermeidung eines operativen Eingriffs (AWMF online 2009, Grivas et al. 2003, Kotwicki et al. 2002, Lonstein 2000, Nachemson und Peterson 1995, Rigo et al. 2003, Weiß et al. 2003, Weiß et al. 2006). Deshalb mussten in der Vergangenheit bei Kindern im Wachstumsalter operative Verfahren wie die ventralen und/oder dorsalen Fusionen, Epiphyseodesen und Arthrodesen angewandt werden. Zudem gab es den Versuch, eine Skoliose mit dem von Paul Harrington entwickelten growing rod aufzudehnen (Andrew und Piggott 1985, Letts und Bobechko 1974, Marks et al. 1996, McMaster 1998, Moe et al. 1984, Rinsky et al. 1985, Tello 1994, Winter 1973, Winter 1981, Winter und Moe 1982, Winter et al. 1968). Durch alle Eingriffe mit Ausnahme des growing-rod-Verfahrens wurde die Wirbelsäule versteift, was sich negativ auf das Körper-, Thorax- und infolgedessen auf das Lungenwachstum auswirken kann.

Eine posteriore Versteifung der Wirbelsäule beim Kind bedingt beim erwachsenen Patienten eine viel zu kurze Wirbelsäule und kann zum crankshaft phenomenon führen, das eine aufgrund des weiterhin bestehenden anterioren Wachstums allmähliche Rotation und Deformierung der Wirbelsäule und des Rumpfes beinhaltet (Dubousset et al. 1989, Hefti und McMaster 1983). Bisher ist jedoch keine abschließende Bewertung möglich, wie häufig ein crankshaft phenomenon bei der alleinigen dorsalen Fusion von sehr jungen Patienten mit offener Wachstumsfuge (Risser-Stadien 0 oder 1) auftritt (Hopf 2000). Diesem Problem vorzubeugen gelingt durch eine vorab durchgeführte anteriore Fusion, wodurch das Wachstum der Wirbelsäule nahezu gänzlich verhindert wird (Shufflebarger und Clark 1991). Ferner entsteht aufgrund einer anterioren oder posterioren Versteifung ein vermindertes 
Wachstum des Thorax. Durch den Platzmangel im Brustkorb entsteht eine Alteration der Lunge, die mangels Lungensubstanz im thoracic insufficiency syndrome, welches definiert ist als das Unvermögen des Thorax, eine adäquate Respiration bzw. normales Lungenwachstum zu gewährleisten, oder sogar im Lungenversagen enden kann (Campbell und Smith 2007, Campbell et al. 2003, Hell et al. 2005 a).

Zur Behandlung dieser progressiven Deformitäten von Thorax und Wirbelsäule im Kindesalter stehen konservative und operative Möglichkeiten zur Verfügung. Eine konservative Therapieoption ist das Tragen eines Korsetts. Dies kann jedoch die Entwicklung des Kindes erheblich - besonders durch eine Thoraxkompression und vermindertes Lungenwachstum - stören (McCarthy et al. 2000).

Das Ziel einer modernen Behandlung bei Kindern mit Anomalien der Wirbelsäule und Skoliose sollte deshalb sein, dass neben der Wiederherstellung der mechanischen Abweichung das Wachstumspotenzial der Wirbel gefördert wird und sowohl die Verbesserung des Volumens, der Symmetrie und der Funktion des Thorax als auch eine Korrektur der Skoliose verfolgt werden (Akbarnia 2007).

In den letzten Jahren haben sich zwei operative Verfahren zur Behandlung von earlyonset-Skoliosen (null bis fünf Jahre) durchgesetzt (Akbarnia und Emans 2010, Yazici und Emans 2009). Zum einen kann die growing-rod-Technik und zum anderen eine Expansionsthorakoplastik mit Implantation des VEPTR (vertical expandable prosthetic titanium rib)-Instrumentariums angewandt werden. Viele Autoren empfehlen, ein growing-rod-System zu implantieren, wenn das primäre Problem die Wirbelsäule betrifft (Akbarnia und Emans 2010, Thompson et al. 2007, Yazici und Emans 2009). Sind beim Patienten hingegen zusätzlich eine Thoraxdeformität und/oder ein thoracic insufficiency syndrome vorhanden, so hat sich das VEPTRVerfahren bewährt (Akbarnia und Emans 2010, Cahill et al. 2010, Debnath 2010, Emans et al. 2005, Hell et al. 2005 b, Thompson et al. 2007, Yazici und Emans 2009). Insbesondere wenn das Kind sehr jung ist und eine lange Wachstumsphase vor sich hat, ist das VEPTR-Verfahren bei kongenitaler Skoliose mit Thoraxfehlbildungen überlegen. Besteht die kongenitale Skoliose ohne Thoraxanomalien, so scheinen beide Verfahren etwa gleichwertig zu sein (Yazici und Emans 2009). 
Es wurde gezeigt, dass die Lungenfunktion sowohl durch den VEPTR als auch wahrscheinlich durch Implantation eines growing rods verbessert werden kann (Akbarnia et al. 2005, Jiang et al. 2011, Motoyama et al. 2009). Nach der Expansionsthorakoplastik und VEPTR-Implantation wird das Brustkorbvolumen und somit der Platz für die Lunge größer (Yazici und Emans 2009). Beim growing rod muss meistens postoperativ für sechs Monate ein Korsett getragen werden, welches bei der Behandlung mit dem VEPTR entfällt (Akbarnia et al. 2005, Campbell et al. 2004 a, Hasler et al. 2010). Man geht davon aus, dass sich eine korsettfreie Nachbehandlung positiv auf Thorax und Lunge auswirkt.

\subsection{Problem der Ossifikation}

\subsubsection{Inzidenz und Lokalisation}

Die bisherige Annahme war, dass bei der Behandlung mit dem vertical expandable prosthetic titanium rib (VEPTR) aufgrund der geringeren Manipulation an der Wirbelsäule seltener Ossifikationen bzw. Fusionen auftreten als beim growing-rodVerfahren (Yazici und Emans 2009).

Anhand der vorliegenden Studie konnte gezeigt werden, dass 24\% der Kinder $(n=13)$, die mit dem VEPTR versorgt wurden, auf dem Röntgenbild erkennbare Ossifikationen im Untersuchungszeitraum entwickelt hatten. Werden zwei Patienten nicht berücksichtigt, die im Untersuchungszeitraum nur eine VEPTR-PrimärImplantation erhalten hatten, bekamen 25\% $(n=13)$ der Patienten $(n=52)$ eine Ossifikation. In Analysen von Yazici und Emans bzw. Akbarnia und Emans wurde geschildert, dass Wirbelfusionen während der Behandlung mit dem VEPTR sehr selten auftraten (Akbarnia und Emans 2010, Yazici und Emans 2009). Dies könnte darin begründet sein, dass der VEPTR die Bewegung im Brustkorb zulässt und somit thorakale Wirbelfusionen verhindert (Sankar et al. 2010). Refusionen der Rippen stellten sich etwas häufiger bei Patienten, die aufgrund kongenitaler Skoliosen und Rippenfusionen mit dem VEPTR behandelt wurden, in der Computertomografie dar. Leider wurden keine genauen Daten erfasst (Akbarnia und Emans 2010).

Die Autoren Akbarnia und Emans erwähnten, dass entlang des VEPTR liegende Rippen durch Kalzifikationen und Ossifikationen fusionieren könnten und dies wahrscheinlich in einer ähnlichen Häufung wie die Autofusionen der Wirbelsäule 
nach growing-rod-Implantation vorkommen könnte. Bei Akbarnia und Emans bzw. Emans et al. wurde das Problem der Refusion der Rippen nur bei 9\% bzw. 6\% der Patienten beschrieben (Akbarnia und Emans 2010, Emans et al. 2005, Yazici und Emans 2009). Die in dem vorliegenden Kollektiv gefundene Rate an Ossifikationen ist mit $24 \%$ um einiges höher als bisher angenommen.

Das Prinzip und die Indikationen des VEPTR und des growing rods sind verschieden und daher ein Vergleich der beiden Verfahren schwierig (Debnath 2010). Dennoch scheint es sinnvoll, die Komplikationen inklusive der Rate an Ossifikationen zu vergleichen, da bei beiden Methoden etwa zwei bis drei Verlängerungsoperationen pro Jahr erfolgen. So zeigten die Analysen von Akbarnia und Emans bzw. Yazici und Emans, dass im Gegensatz zum VEPTR beim growing rod häufig spontane Wirbelfusionen entstanden (Akbarnia und Emans 2010, Yazici und Emans 2009). In einer Untersuchung von Cahill et al. bekamen acht von neun Patienten (89\%) eine ungewollte vorzeitige Wirbelfusion (Cahill et al. 2010). Demnach entstanden beim growing rod (89\%) viel häufiger Ossifikationen bzw. die gravierende Problematik der Wirbelsäulenfusionen als in unserer Studie beim VEPTR (24\%).

Das Entstehen von spontanen Ossifikationen scheint multifaktoriell erklärbar zu sein. Es wird angenommen, dass als Voraussetzung eine Verletzung des Periosts vorliegen muss. In einer Studie von Martinez et al. war bei einer induzierten nicht dislozierten Ermüdungsfraktur der Ulna in einer Maus an der Stelle des Periostdefekts bereits nach einer Woche zentral Knorpel mit umgebendem Lamellenknochen (Kallus) entstanden. Auf diese Weise erlangte der Knochen die in der Situation bestmögliche Stabilität, die nach zwei Wochen im Idealfall dem Zustand vor der Verletzung glich. Diese Antwort des verletzten Knochens erfolgte abhängig vom Schweregrad der aufgrund der mechanischen Beanspruchung bedingten Schädigung. Die Faktoren, die diese Bildung des Lamellenknochens regulieren, sind bisher kaum verstanden (Martinez et al. 2010, Uthgenannt et al. 2007). Risikofaktoren für eine solche Periostverletzung könnten bei der Behandlung mit dem VEPTR-Implantat, das ein den Thorax stabilisierendes flexibles Verfahren darstellt, die vermehrte Bewegung im System und der verstärkte Druck auf Knochenstrukturen an den Verankerungspunkten der Implantate mit den daraus resultierenden Implantat-Wanderungen sein. Ein weiterer Risikofaktor könnte der durch Distraktion 
erhöhte Druck und Zug auf die Knochenstrukturen sein, die durch das Implantat aufgedehnt bzw. überbrückt werden.

Die Lokalisation der Ossifikationen bestätigt die Annahme der Abhängigkeit des Entstehens einer Verknöcherung von der Periostverletzung. Die meisten Ossifikationen bildeten sich an der Lendenwirbelsäule (54\%), obwohl dort die wenigsten Implantate verankert wurden (sieben Ossifikationen bei 31 Implantaten; 23\%). Es folgten sowohl der Beckenbereich mit 23\% (drei bei 52; 6\%) als auch der Rippenbereich mit 23\% (drei bei 106; 3\%).

Hervorzuheben ist, dass am Beckenkamm nur auf der konkaven Seite der Skoliose Ossifikationen entstanden. Dies könnte dadurch bedingt sein, dass durch das Implantieren des longitudinalen Distraktionsimplantats (VEPTR) der oberhalb der kaudalen Implantatverankerung liegende Anteil des konkavseitigen Beckenknochens einer verminderten Drucklast oder sogar Zugkräften ausgesetzt ist. Das Wachstum könnte durch den Hueter-Volkmann-Mechanismus erklärt werden. Dieser beschreibt, dass ein vermehrter Druck auf die Epiphyse ein reduziertes Wachstum und ein verminderter Druck eine Wachstumszunahme bewirkt (Hueter 1862, Volkmann 1869). Durch die Implantatabstützung erfolgt eine Druckentlastung für die Wirbelsäule generell.

Die hohe Anzahl an Ossifikationen an der Lendenwirbelsäule ergab sich vermutlich aus zwei Gründen: Zum einen waren die Implantate an der Lamina des Wirbelbogens verankert und verursachten daher einen sehr kleinen Druckpunkt; zum anderen war die Wanderung der Implantate an der Lendenwirbelsäule am stärksten. Dies scheint darin begründet, dass viel Bewegung und daraus resultierend eine vermehrte Wanderung der Implantate in diesem VEPTR-System vorhanden war, da vor allem gehfähige Kinder mit kongenitaler, sehr steifer Skoliose dieses System implantiert bekommen hatten (Campbell und Smith 2007, Hell 2004, McMaster und Ohtsuka 1982). Sowohl der kleine Druckpunkt als auch die vermehrte ImplantatWanderung steigern wahrscheinlich die Gefahr einer Periostverletzung am Wirbelbogen der Lendenwirbelsäule.

Im Gegensatz zum Lendenwirbelsäulenbereich wurde am Beckenkamm weniger Reaktion am Knochen verursacht. Im Allgemeinen ossifizierten die Implantate aufgrund der Lendenwirbelsäulenverankerungen an den kaudalen Befestigungen viel 
häufiger als an den kranialen Rippenverankerungen. Die Elastizität bzw. Beweglichkeit der Rippen bewirkt ein Nachgeben. Dies scheint einen protektiven Effekt auf mögliche Periostverletzungen zu haben, sodass weniger Ossifikationen entstehen.

Die bereits beschriebene Annahme, dass Ossifikationen an der am stärksten gefährdeten Struktur für Periostdefekte auftreten, wurde durch die Analyse der einzelnen Implantate bekräftigt. So ossifizierten die Hauptimplantate „Rippe zu LWS auf der konkaven Seite“ (26\%) und „Rippe zu Becken auf der konkaven Seite“ (13\%), die jeweils den größten Teil der Abstützung am Skelett leisten, prozentual erheblich mehr als die akzessorischen Implantate „Rippe zu Rippe auf der konkaven Seite“ (4\%) und „Rippe zu Becken auf der konvexen Seite“ (0\%).

Beim Vergleich der Lokalisation von Ossifikationen bzw. Fusionen beim growing rod und dem VEPTR ist hervorzuheben, dass sich der Ort der Verknöcherung fast immer unterschied. So betraf die Ossifikation beim growing rod vorwiegend die Wirbelsäule in Form von Wirbelfusionen (Akbarnia und Emans 2010, Cahill et al. 2010, Emans et al. 2005, Yazici und Emans 2009). Im Gegensatz dazu ossifizierten beim VEPTRVerfahren neben den Rippen entlang der Implantate hauptsächlich die Bereiche der Implantatverankerungen. Hierbei waren die kaudalen Befestigungen aufgrund der Lendenwirbelsäulenverankerungen viel häufiger betroffen als die kranialen.

\subsubsection{Zeitpunkt und Wahrscheinlichkeit}

Unter den Spezialisten der nicht fusionierenden Operationsverfahren für early-onsetSkoliosen wurde bisher - ohne wissenschaftlichen Nachweis - übereinstimmend angenommen, dass beim VEPTR-Verfahren Ossifikationen seltener und später als bei growing-rod-Verfahren auftreten würden (Hell 2011). Ein Anzeichen dafür liefert ein Einzelfall in einer Analyse von Akbarnia und Emans, in dem nach sieben Jahren Behandlungsdauer erstmals eine Ossifikation auftrat. Nach operativer Entfernung war auch nach weiteren vier Jahren Therapie kein Rezidiv entstanden (Akbarnia und Emans 2010). In unserer Untersuchung wurde gezeigt, dass Implantate zu einem früheren Zeitpunkt ossifizierten. So traten bei 19\% in den ersten 24 Monaten und bei $\mathbf{1 1} \%$ der Patienten sogar in den ersten zwölf Monaten Ossifikationen auf. Die Rate an Verknöcherungen nahm im Verlauf der Behandlung nicht zu. So ossifizierten jeweils $12 \%$ der Implantate ab dem 13. bis einschließlich 24. Monat und ab dem 25. 
bis einschließlich 48. Monat. In einer ausgedehnten Literaturrecherche konnte keine analoge Studie zum growing-rod-Verfahren gefunden werden.

Die Verlängerungsoperationen erfolgten individuell bei den Patienten in variablen Abständen zwischen drei und 14 Monaten. Die Operationen sind mit einer Manipulation an den Implantaten verbunden. Aus diesem Grund sollte des Weiteren verifiziert werden, nach wie vielen Operationen Ossifikationen auftraten. Die Rate an Fusionen war ab der siebten bis einschließlich neunten Operation (21\%) am höchsten. So fand annähernd eine Verdopplung im Vergleich zu den Zeiträumen ab der ersten bis einschließlich dritten Operation (13\%) und ab der vierten bis einschließlich sechsten Operation (11\%) statt. Daher muss das Ziel sein, mit möglichst wenigen Verlängerungsoperationen auszukommen, was natürlich in erster Linie wegen der bekannten Operationskomplikationen angestrebt wird.

Bei der Analyse, inwieweit Patienten keine Ossifikation bekamen, zeigte sich, dass nach 21 Monaten Behandlungszeit 74\% der Patienten nicht ossifiziert waren und nach 53 Monaten 52\% der Kinder bis zum Ende oder dem aktuellen Stand ihrer Behandlung ohne Verknöcherung blieben. Analog dazu entwickelte sich nach vier Operationen bei 75\% der Patienten keine Fusion und nach neun Operationen blieben $54 \%$ der Kinder ohne Ossifikation. Auch hier ließ sich keine vergleichbare Analyse des growing-rod-Verfahrens recherchieren.

Anhand des Patientenkollektivs wurde die Wahrscheinlichkeit für ein Kind berechnet, eine Ossifikation im Verlauf der Behandlung mit dem VEPTR zu bekommen. Auf diese Weise wäre es in Zukunft möglich, vor Beginn der Anwendung des VEPTRVerfahrens mitzuteilen, wie hoch das Risiko im Verlauf der Behandlung ist, dass bei einem Kind Ossifikationen an einem Implantat und die damit verbundenen Probleme auftreten könnten. Das Risiko in unserem Kollektiv war 11\% in den ersten zwölf Monaten und jeweils $10 \%$ ab dem 13. bis einschließlich 24. Monat, ab dem 25. bis einschließlich 48. Monat und bei über 48 Monaten Behandlungsdauer. Analog dazu lag das Risiko für ein Kind ab der ersten bis einschließlich dritten Operation bei 13\%, ab der vierten bis einschließlich sechsten Operation bei $10 \%$ und ab der siebten bis einschließlich neunten Operation bei 17\% eine Ossifikation zu bekommen. 


\subsubsection{Größe der Ossifikationen im Verlauf der Behandlung}

Die Größen der Ossifikationen bei den 13 betroffenen Patienten betrugen in unserem Kollektiv durchschnittlich $400 \mathrm{~mm}^{2}$ (48 bis 1664) und waren sowohl bei erstmaligem Auftreten als auch im Verlauf sehr variabel. Im Mittel nahmen die Verknöcherungen nach einer Verlängerungsoperation um $44 \%$ ( 0 bis 170 ) auf $574 \mathrm{~mm}^{2}$ (51 bis 2624) und nach 3,9 Operationen (im Mittel letztmalig gemessene Ossifikation) um 168\% (19 bis 1590) auf $1071 \mathrm{~mm}^{2}$ (75 bis 3399) zu. Die recherchierte Literatur wies keine vergleichbare Analyse für das VEPTR- bzw. das growing-rod-Implantat auf.

Eine Schwäche der vorliegenden Untersuchung war die Messgenauigkeit. Zum einen konnten einige Röntgenaufnahmen aufgrund von Überlagerungen (vor allem der Darmschlingen) nicht konklusiv beurteilt werden, zum anderen konnte anhand der Röntgenbilder das dreidimensionale Ausmaß der Ossifikationen nicht in allen Ebenen ermittelt werden.

\subsubsection{Einflussgrößen auf die Ausbildung von Ossifikationen}

Vermeintliche Einflussgrößen wie das Geschlecht, die Grunderkrankung, das VEPTR-System, das Alter bei der Primär-Implantation und der Cobb-Winkel prä bzw. post Primär-Implantation hatten in unserem Patientenkollektiv im Mittel keinen signifikanten Einfluss darauf, ob ein Kind eine Ossifikation bekam oder nicht.

Dagegen zeigte sich bei Patienten mit einer Ossifikation im Mittel eine signifikant kleinere Differenz (24,7 Grad zu 36,3 Grad) der Cobb-Winkel prä und post PrimärImplantation, als bei Kindern, die keine Verknöcherung bekamen. Dies waren zum Großteil die Kinder mit kongenitalen Skoliosen, wo durch eine ausgedehnte Rippentrennung eine großflächige Periostverletzung stattfand und bei den rigiden Krümmungen auch mehr Druck auf dem Implantat lastete. Die Kinder mit großer Korrektur der Skoliose hatten im Normalfall sehr weiche und flexible Kurven, die sich ohne große Drucklast gut aufrichten ließen. 


\subsubsection{Vergleich der Behandlungsdauer, der Operationsanzahl und der Komplikationen}

Patienten, die eine Ossifikation bekamen, hatten in unserem Patientenkollektiv durchschnittlich eine signifikant längere Behandlungsdauer (48,3 zu 24 Monate) und signifikant mehr Operationen (9,2 zu 4,9 Operationen) als Kinder ohne Verknöcherung. Hier ist jedoch anzumerken, dass die Ossifikation die längere Behandlungsdauer bzw. die größere Operationsanzahl nicht bedingen muss. Vielmehr scheint sich dieses Ergebnis dadurch zu ergeben, dass viele Patienten aufgrund einer sehr geringen Anzahl an Verlängerungsoperationen bzw. einer sehr kurzen Behandlungsdauer während des Untersuchungsintervalls keine Ossifikation aufwiesen. Auf diese Weise wird ein signifikantes Ergebnis vorgetäuscht.

Im Hinblick auf die Komplikationen wie Implantat-Bruch, -Infektion und -Ausriss bestand in unserem Patientenkollektiv kein signifikanter Unterschied zwischen Patienten, die eine Ossifikation bekamen und denen, die keine aufwiesen.

\subsubsection{Folgen von entstandenen Ossifikationen}

Die Folgen der Ossifikationen könnten vielfältig sein. Allerdings ist noch nicht geklärt, ob sie eine klinische Bedeutung haben. Entwickelt sich eine Ossifikation im Rippenbereich, so entsteht erst ein klinisches Problem, wenn die Verlängerung des VEPTR oder das Wachstum des Brustkorbes behindert wird. Des Weiteren kann sich die unbeabsichtigte Versteifung der Brustwand langfristig negativ auf die Brustwandmechanik auswirken (Akbarnia und Emans 2010). Dies könnte zur erschwerten sekundären Atmung und darüber hinaus bis zum thoracic insufficiency syndrome führen, welches das Unvermögen des Thorax ist, eine adäquate Respiration bzw. normales Lungenwachstum zu gewährleisten (Campbell et al. 2003). Der Schweregrad dieser Komplikation hängt vermutlich von der Größe der Ossifikation ab. Je mehr Rippen miteinander verwachsen sind, desto rigider ist der Brustkorb.

Entsteht eine Ossifikation an der Lendenwirbelsäulenverankerung eines Implantats, so könnte diese ähnlich der posterioren Wirbelsäulenversteifung ein crankshaft phenomenon bewirken (Dubousset et al. 1989, Hefti und McMaster 1983). Dieses beinhaltet aufgrund des weiterhin bestehenden anterioren Wachstums eine 
allmähliche Rotation und Deformierung der Wirbelsäule und des Rumpfes. Das Ausmaß dieser Komplikation wird vermutlich vom Patientenalter bei Auftreten und von der Größe der Ossifikation beeinflusst. Als weiterer Negativaspekt muss bei Ossifikationen im Lendenwirbelsäulenbereich unter Umständen bei der definitiven Versorgung mit einer dorsalen Spondylodese eine längere Fusionsstrecke gewählt werden.

\subsection{Problem der Implantat-Wanderung}

\subsubsection{Implantat-Wanderung und Ossifikation}

Die Implantat-Wanderung scheint wie beschrieben über den Weg der Periostverletzung mittelbar ein Risikofaktor für Ossifikationen zu sein. Durch die Implantat-Wanderung entstehen Druck und Reibung am Knochen, welches zur Zerstörung von Periost führen könnte. Dies würde wiederum das Entstehen spontaner Ossifikationen bewirken (Martinez et al. 2010). Aus diesem Grund wurden neben den Ossifikationen auch die Wanderungen der Implantate durch den Knochen analysiert.

\subsubsection{Inzidenz und Lokalisation}

Durch vermehrte Bewegung in einem flexiblen System wie dem VEPTR-Konstrukt werden wahrscheinlich viele Implantat-Wanderungen hervorgerufen. Vermutlich aus diesem Grund wanderte das VEPTR-Instrumentarium bei 93\% der Patienten des Kollektivs vom ursprünglichen Verankerungspunkt ausgehend durch den Knochen. Nur bei $7 \%$ der Kinder $(n=4)$, die ausnahmslos höchstens eine Verlängerungsoperation im Untersuchungszeitraum bekommen hatten, wanderte kein Implantat. Folglich konnte bestätigt werden, dass bei nahezu allen Patienten im Verlauf einer längeren Behandlung Implantate durch den Knochen wanderten bzw. durch Druck eine Knochendeformierung bewirkten. Wie auch von Akbarnia und Emans in ihrer Untersuchung beschrieben, erfolgte diese Implantat-Wanderung sehr langsam und blieb bei einem Großteil der Patienten asymptomatisch (Akbarnia und Emans 2010). Im vorliegenden Kollektiv fand bei $19 \%$ der Patienten ein ImplantatAusriss statt, der in der Regel zur Instabilität des VEPTR-Konstrukts führte. Campbell 
und Smith beschrieben in ihrer Untersuchung mit einem großen Kollektiv von 201 Patienten, dass nur 27\% der Implantate durch den Knochen wanderten. In dieser Studie wurden jedoch nur Implantate berücksichtigt, bei denen das Verankerungselement im Verlauf den gesamten Knochen durchwandert hatte (Campbell und Smith 2007). Auf gleiche Weise entstanden die Raten von 14\% bzw. $31 \%$ Implantat-Wanderungen bei den Analysen zur growing-rod-Technik (Klemme et al. 1997, Tello 1994). Die vorliegenden Daten mit einer 100\%igen ImplantatWanderung nach mindestens einem Jahr Behandlung sind daher nicht identisch mit den Daten zum Implantat-Ausriss bzw. kompletten Durchwanderung zu sehen.

Bei der Analyse der unterschiedlichen Implantate zeigte sich anhand des Patientenkollektivs, dass die Implantate „Rippe zu Becken“ zu etwa 80\% jeweils kranial durch die Rippen wanderten. Das kaudale Ende schien im massiven Beckenknochen solider verankert zu sein und wanderte geringer als die kranialen Elemente. Im Gegensatz zu unserem Ergebnis beschrieben Akbarnia und Emans, dass das kaudale Ende des Implantats „Rippe zu Becken“ besonders bei unilateraler Verwendung wanderte (Akbarnia und Emans 2010). Eine gelegentliche Wanderung der Implantate „Rippe zu Becken“ durch den Beckenknochen schilderten Campbell et al. (Campbell et al. 2004 a).

Die Implantate „Rippe zu LWS auf der konkaven Seite“ wanderten in 13\% der Fälle nur kranial und in 3\% der Fälle nur kaudal, hingegen zu 77\% an beiden Enden. Der Druck auf den Wirbelbogen des Lendenwirbelkörpers wurde folglich in vier von fünf Fällen so ungünstig durch die kaudale Verankerung übertragen, dass der Wirbelbogen diesem Druck nicht standhielt. Kleine Kinder sind im Hinblick auf Implantat-Wanderungen besonders gefährdet, da die Knochenstärke bei Kindern geringer ausgeprägt ist als bei Erwachsenen. Ab Ende der Pubertät weisen Jugendliche eine peak bone mass auf, welche definiert ist als die Menge an Knochengewebe nach der skelettalen Ausreifung (Bonjour et al. 1994). Ferner wird das Risiko für Implantat-Wanderungen für Kinder noch größer, je kleiner der Druckpunkt des Implantats am Knochen ist.

Durch Teile der Rippen wanderten die Implantate „Rippe zu LWS auf der konkaven Seite" sogar in neun von zehn Fällen, obwohl die Rippen wegen ihrer Beweglichkeit dem Druck durch Deformierung nachgeben. Andererseits haben die Rippen bei sehr jungen Kindern eine weichere Knochenstruktur als die Wirbelbögen. Dies begünstigt 
wiederum eine Wanderung des Implantats. Insgesamt scheint der letztgenannte Aspekt zu überwiegen. Diese Annahme wird dadurch bekräftigt, dass das Implantat „Rippe zu Rippe auf der konkaven Seite“ zu 91\% (43\% an beiden Enden; 35\% kranial; 13\% kaudal) wanderte.

\subsubsection{Strecke im Verlauf der Behandlung}

Die Implantate „Rippe zu Rippe auf der konkaven Seite“ wanderten in unserem Patientenkollektiv am zweithäufigsten (91\%), dafür aber die geringste Strecke. Die anderen Implantate wanderten durchschnittlich signifikant um verschiedene Faktoren mehr. Am häufigsten (93\%) und am stärksten wanderten im Mittel die Implantate „Rippe zu LWS auf der konkaven Seite“ (Faktor 4,5 bzw. 8), gefolgt von den Implantaten „Rippe zu Becken auf der konvexen Seite“ (2,5 bzw. 6) und den Implantaten „Rippe zu Becken auf der konkaven Seite“ (2 bzw. 5).

Werden nur die Implantate „Rippe zu Becken“ miteinander verglichen, so wanderten die Hauptimplantate auf der konkaven Seite signifikant um den Faktor 1,5 stärker als die akzessorischen Implantate auf der konvexen Seite. Dies spiegelt das bereits beschriebene Problem der ungleichmäßigen Druckverteilung wieder. Ferner wanderten im Mittel die Hauptimplantate "Rippe zu LWS auf der konkaven Seite“ signifikant um den Faktor 2,75 stärker als die akzessorischen Implantate „Rippe zu Rippe auf der konkaven Seite“.

Die Implantate „Rippe zu LWS auf der konkaven Seite“ wanderten wahrscheinlich aus drei Gründen durchschnittlich stärker als die Implantate „Rippe zu Becken auf der konkaven Seite“. Sie wurden in dem System „VEPTR konkav einseitig“ implantiert, das der vermutlich stärkeren Bewegung ausgesetzt war, da dieses vor allem bei gehfähigen Kindern mit kongenitaler, sehr steifer Skoliose verwendet wurde (Campbell und Smith 2007, Hell 2004, McMaster und Ohtsuka 1982). Des Weiteren wanderten die Implantate „Rippe zu Becken auf der konkaven Seite“ am kaudalen Ende vermutlich wegen der geringeren Drucklast wenig oder gar nicht. Zudem schienen die akzessorischen Implantate „Rippe zu Becken auf der konvexen Seite“ im Vergleich zu den akzessorischen Implantaten „Rippe zu Rippe auf der konkaven Seite" die Aufgabe der Hauptimplantate, die Wirbelsäule zu stabilisieren, stärker zu unterstützen. Die Implantate „Rippe zu Rippe auf der konkaven Seite“ dienten hingegen vielmehr dazu, den Thorax zu stabilisieren (Campbell und Smith 2007, Hell 
et al. 2004). Diese Annahme wird dadurch bekräftigt, dass der Unterschied der signifikant stärkeren Wanderung des Hauptimplantats im Vergleich zum akzessorischen Implantat im System „VEPTR konkav einseitig“ (Faktor 2,75) fast doppelt so groß war wie im System „VEPTR beidseitig“ $(1,5)$.

In beiden Systemen kam es parallel zur Wanderung des Hauptimplantats immer zu einer signifikanten Wanderung des akzessorischen Implantats. Dies bedeutet, dass in einem VEPTR-System mit mindestens zwei Implantaten niemals eins allein wanderte. Anders formuliert: Wenn eine Implantat-Wanderung stattfand, war immer das gesamte VEPTR-Konstrukt in Bewegung.

Das Hauptimplantat „Rippe zu Becken auf der konkaven Seite“ wanderte im Mittel signifikant um den Faktor zwei stärker im System „VEPTR Becken konkav einseitig“ als im System „VEPTR beidseitig“. Dagegen bestand kein signifikanter Unterschied darin, ob das Hauptimplantat „Rippe zu LWS auf der konkaven Seite“ im System „VEPTR konkav einseitig“ allein oder zusammen mit einem akzessorischen Implantat verwendet wurde. Beim Vergleich der beiden Hauptimplantate miteinander wanderte das Implantat „Rippe zu LWS auf der konkaven Seite“ durchschnittlich signifikant um den Faktor zwei stärker als das Implantat „Rippe zu Becken auf der konkaven Seite“.

Diese Ergebnisse scheinen auf folgendes schließen zu lassen: Das System „VEPTR beidseitig“ verteilt den Druck auf die einzelnen Implantate gleichmäßiger als das System „VEPTR konkav einseitig“ und die Beckenverankerung im System „VEPTR beidseitig“ ist entgegen der bisherigen Annahme stabiler als die Befestigung am Wirbelbogen des Lendenwirbelkörpers im System „VEPTR konkav einseitig“.

\subsubsection{Einflussgrößen auf das Entstehen der Implantat-Wanderung}

Vermeintliche Einflussgrößen auf die Implantat-Wanderung wie das Geschlecht, die Grunderkrankung, das Alter bei der Primär-Implantation, der Cobb-Winkel prä und post Primär-Implantation und die Differenz der Cobb-Winkel prä und post PrimärImplantation erwiesen sich in unserem Patientenkollektiv bis auf eine Ausnahme als nicht signifikant. Das Implantat „Rippe zu Becken auf der konkaven Seite“ wanderte im Mittel um den Faktor zwei signifikant stärker bei den $50 \%$ der Patienten, die den größeren Winkel prä Primär-Implantation hatten. Vermutlich entfachte das 
Aufdehnen durch das Einbringen des Implantats bei größerem Cobb-Winkel prä Primär-Implantation einen stärkeren Druck auf den Knochen.

\subsection{Sonstige Komplikationen}

Bei 9\% der Patienten ( $n=5)$ musste in dem Kollektiv ( $n=57)$ ein Implantat aufgrund einer Infektion vorzeitig explantiert werden. In vergleichbaren Studien von Campbell et al. und Hasler et al. mit Kindern, die ebenfalls eine VEPTR-Implantation erhalten hatten, war die Rate an Infektionen mit 11\% bzw. 22\% höher (Campbell et al. 2004 b, Hasler et al. 2010). Die growing-rod-Verfahren wiesen ähnliche Raten auf (Akbarnia et al. 2005, Klemme et al. 1997, Tello 1994).

Bei $19 \%$ der Patienten $(n=11)$ riss in dem Kollektiv $(n=57)$ jeweils ein Implantat aus seiner Verankerung aus. Bei Hasler et al. dislozierten 16\% aller Fixationen und 19\% der kranial an den Rippen befestigten Ringe (Hasler et al. 2010). Analog rissen in der Studie von Campbell et al. bei $26 \%$ der Patienten kraniale Verankerungen aus (Campbell et al 2004 b). Im Vergleich zum VEPTR hatten beim growing rod 14\% der Patienten einen Implantat-Ausriss bzw. 28\% eine Implantat-Dislokation an der kranialen und 3\% an der kaudalen Befestigung (Klemme et al. 1997, Tello 1994). Bei der Analyse der modifizierten growing-rod-Technik, dem Hybridinstrumentarium (kaudale Verankerung an der Lendenwirbelsäule; kraniale Verankerung an der Rippe), zeigte sich bei Sankar et al. eine signifikant niedrigere Komplikationsrate als beim VEPTR-Verfahren. Laut Sankar et al. könnte dies zum Teil darauf zurückgeführt werden, dass bei dem Hybridinstrumentarium ein Lendenwirbelsäulenhaken als Rippenverankerung verwendet wird. Dieser hat eine breitere Kontaktfläche an der Rippe als der VEPTR und führe daher aufgrund der besseren Druckverteilung zu weniger Implantat-Ausrissen und infolgedessen zu weniger Komplikationen (Sankar et al. 2010).

In unserer Untersuchung betraf die Dislokation als weitere Differenzierung nur die Hauptimplantate der VEPTR-Systeme. Die Implantate „Rippe zu LWS auf der konkaven Seite“ rissen in 23\% der Fälle und die Implantate „Rippe zu Becken auf der konkaven Seite“ in $13 \%$ der Fälle aus. Dies bekräftigt die bereits beschriebene Annahme, dass die Implantate „Rippe zu Becken auf der konkaven Seite“ wahrscheinlich stabiler verankert waren und somit dem Druck besser standhielten. 
Patienten, bei denen ein Implantat ausriss, hatten einen signifikant größeren CobbWinkel prä bzw. post Primär-Implantation. Der Unterschied betrug $17,7^{\circ}$ bzw. 23,1 zu den Patienten, bei denen kein Implantat dislozierte. Dies bedeutet, dass die Kinder zum frühestmöglichen Zeitpunkt mit einem kleinen Cobb-Winkel operiert werden sollten, was natürlich vor allem wegen der progressiven Skoliose der Patienten geschieht. Es ist fraglich, ob der Unterschied der Cobb-Winkel von etwa $20^{\circ}$ zwischen beiden Gruppen als signifikanter Effekt auf einen Implantat-Ausriss gewertet werden kann. So wurde zwar in einer Studie von Facanha-Filho et al. festgestellt, dass bei erfahrenen Untersuchern die Messgenauigkeit bei der CobbMethode bis zu $6^{\circ}$ differiert, jedoch in einer Studie von Loder et al. bis zu $19^{\circ}$ abweicht (Campbell und Hell-Vocke 2003, Facanha-Filho et al. 2001, Loder et al. 1995).

Bei $7 \%$ der Patienten $(n=4)$ in dem Kollektiv $(n=57)$ trat ein Implantat-Bruch auf. Unter diesen war ein Kind, bei dem zweimal ein Implantat brach. Die Brüche betrafen nur die Hauptimplantate. In anderen Studien wurde die Rate an Implantat-Brüchen als sehr selten beschrieben bzw. mit 6\% beziffert (Campbell und Smith 2007, Yazici und Emans 2009). Dagegen wurden in Untersuchungen des growing rods $18 \%$ bzw. $27 \%$ beschrieben (Klemme et al. 1997, Tello 1994). Bei weiterer Unterteilung traten beim dual growing rod in 14\% der Fälle und beim single growing rod sogar in $29 \%$ der Fälle Implantat-Brüche auf (Thompson et al. 2005).

Nach der primären VEPTR-Implantation wurde bei $91 \%$ der Patienten $(n=51)$ in dem Kollektiv $(n=56)$ die jeweilige Rippe, an der das kraniale Ende des Implantats befestigt wurde, nach oben gespreizt. Dies verdeutlicht, welchen Druck der VEPTR bereits ab Beginn der Behandlung auf die Knochenstrukturen erzeugt. Vergleichbare Studien standen anhand der Literaturrecherche nicht zur Verfügung.

Bei 55 Patienten nahm der Cobb-Winkel von prä zu post Primär-Implantation durchschnittlich signifikant um $45 \%$ von $77,7^{\circ}$ auf $43,3^{\circ}$ ab. Bei der letztmaligen Messung nach im Mittel 4,7 Operationen hatte dieser im Durchschnitt wiederum um $5 \%$ auf $45,5^{\circ}$ zugenommen. Dies entspricht etwa den Ergebnissen vergleichbarer Studien (Hasler et al. 2010, Hell et al. 2005 b, Emans et al. 2005, Ramirez et al. 2009, Wimmer et al. 2010). 
Bei vier Patienten nahm der Kyphose-Winkel von prä zu post Primär-Implantation durchschnittlich signifikant um $32 \%$ von $106,5^{\circ}$ auf $71,5^{\circ}$ ab. Bei der letztmaligen Messung nach im Mittel 7,8 Operationen hatte dieser im Durchschnitt wiederum um $20 \%$ auf $91,5^{\circ}$ zugenommen. Dies entspricht ebenfalls etwa den Ergebnissen vergleichbarer Studien (Hasler et al. 2010, Samdani et al. 2009).

\subsection{Schlussfolgerung und Ausblick}

In der vorliegenden Studie bekamen $\mathbf{2 4} \%$ der Patienten Ossifikationen entlang des VEPTR-Implantats, was eine höhere Rate darstellte als bisher angenommen (Akbarnia und Emans 2010, Yazici und Emans 2009). Im Vergleich zum growing rod entstanden jedoch weniger Ossifikationen (Cahill et al. 2010). Ferner konnte gezeigt werden, dass im Gegensatz zu den Wirbelfusionen beim growing rod die Ossifikationen beim VEPTR entlang des Implantats und vorwiegend an den Verankerungen entstanden (Yazici und Emans 2009).

Zur noch genaueren Messung der Größe der Ossifikationen wäre eine weitere Untersuchung mittels Schnittbilddiagnostik wünschenswert, da dort im Gegensatz zur konventionellen Röntgenbildgebung zwei Probleme entfallen: Zum einen gibt es dort keine Überlagerungsprobleme und zum anderen können die Ossifikationen in allen Ebenen beurteilt werden. Allerdings ist die Computertomografie (CT) aufgrund der wiederholt hohen Strahlenbelastung in der Pädiatrie eine fragliche Option. Die erhöhte Wahrscheinlichkeit von Neoplasien als Langzeitrisiko wurde beschrieben, wahrscheinlich etwa um $0,35 \%$. Durch eine besonders klein eingestellte Strahlendosierung bei CT-Untersuchungen für Kinder kann dieser Prozentsatz eventuell noch minimiert werden (Brenner et al. 2001, Campbell und Hell-Vocke 2003, Donnelly et al. 2001, Paterson et al. 2001). Bei der Magnetresonanztomografie (MRT) hingegen muss der ethische Nutzen gegenüber der Wirtschaftlichkeit abgewogen werden. Die MRT ist zudem nicht die Methode der ersten Wahl zur Darstellung knöcherner Strukturen.

Um zu analysieren, ob Ossifikationen wirklich eine längere Behandlungszeit bzw. mehr Operationen bedingen, sollte weitere Forschung mit einer Patientengruppe stattfinden, bei der als Voraussetzung jedes Kind mindestens zehn 
Verlängerungsoperationen erhalten hat bzw. 48 Monate behandelt worden ist. Dies ist als Langzeitverlaufsstudie wünschenswert.

Durch die vorliegende Arbeit konnte gezeigt werden, dass bei expandierenden Implantaten, selbst wenn sie nicht direkt an der Wirbelsäule verankert sind, durch das Auftreten von Ossifikationen weitreichende medizinische Probleme im Behandlungsverlauf entstehen können. Aufgrund der nur eingeschränkten Fallzahl empfiehlt sich dies in Langzeitstudien zu analysieren.

Ein Risikofaktor der Ossifikation scheint über den Weg der Periostverletzung mittelbar die Implantat-Wanderung zu sein. Diese fand in unserem Kollektiv asymptomatisch bei nahezu allen Patienten statt. Martinez et al. postulierten, dass durch Periostverletzungen Ossifikationen entstehen, wobei die Faktoren, die diese Bildung des Lamellenknochens regulieren, bisher kaum verstanden sind (Martinez et al. 2010, Uthgenannt et al. 2007). Diesen Aspekt betreffend sollte in Zukunft analysiert werden, inwieweit eine Druckerhöhung an den Verankerungspunkten des Implantats durch eine Aufdehnung des Cobb-Winkels bzw. der Skoliose eine Periostverletzung bedingt bzw. Kallusbildung anregt.

Im Rahmen der Implantat-Wanderung kam es zudem bei 19\% der Kinder zu einem Implantat-Ausriss, wobei unsere Ergebnisse darauf hinweisen, dass die Implantatverankerung im Beckenkamm entgegen der bisherigen Annahme stabiler ist als die Abstützung am Wirbelbogen des Lendenwirbelkörpers (Akbarnia und Emans 2010, Campbell et al. 2004 a). Dies scheint vorwiegend darin begründet, dass viel Bewegung und daraus resultierend eine stärkere Wanderung der Implantate in dem System „VEPTR konkav einseitig“ stattfand, da dieses System vor allem bei gehfähigen Kindern mit kongenitaler, sehr steifer Skoliose verwendet wurde (Campbell und Smith 2007, Hell 2004, McMaster und Ohtsuka 1982).

Aufgrund der vorliegenden Studie, die eine höhere als bisher angenommene Rate an Ossifikationen von $24 \%$ präsentierte, wäre es sinnvoll, die Verankerungselemente der Implantate dahingehend zu modifizieren, dass sie den Druck gleichmäßiger auf den Knochen verteilen. Auf diese Weise würde vermutlich der Komplikationsrate inklusive der Implantat-Wanderung, der Periostverletzung und folglich den Ossifikationen vorgebeugt werden.

Des Weiteren zeigte unsere Studie, dass jede Manipulation im Sinne einer weiteren Verlängerungsoperation zur Erhöhung des Risikos für Komplikationen wie Ossifikation, Implantat-Wanderung und -Infektion führt. Man hat das Dilemma, dass 
einerseits bei vielen Operationen diese Probleme auftreten und andererseits, dass bei wenigen Operationen viele Spontanversteifungen entstehen. Deshalb sollten so viele Verlängerungsoperationen wie notwendig, aber so wenige wie möglich, erfolgen. Aus diesem Grund sind Implantate entwickelt worden und aktuell in der klinischen Erprobung, die durch einen Magneten, der dem Patienten auf dem Rücken über dem Implantat aufgelegt wird, extern verlängert werden können.

Abb.35:

Der Ellipse MAGEC ${ }^{\mathrm{TM}}$ Remote Controller zum Auflegen auf den Rücken. Durch Magnetkräfte wird das Implantat verlängert (aus Ellipse Technologies, Inc. 2010).

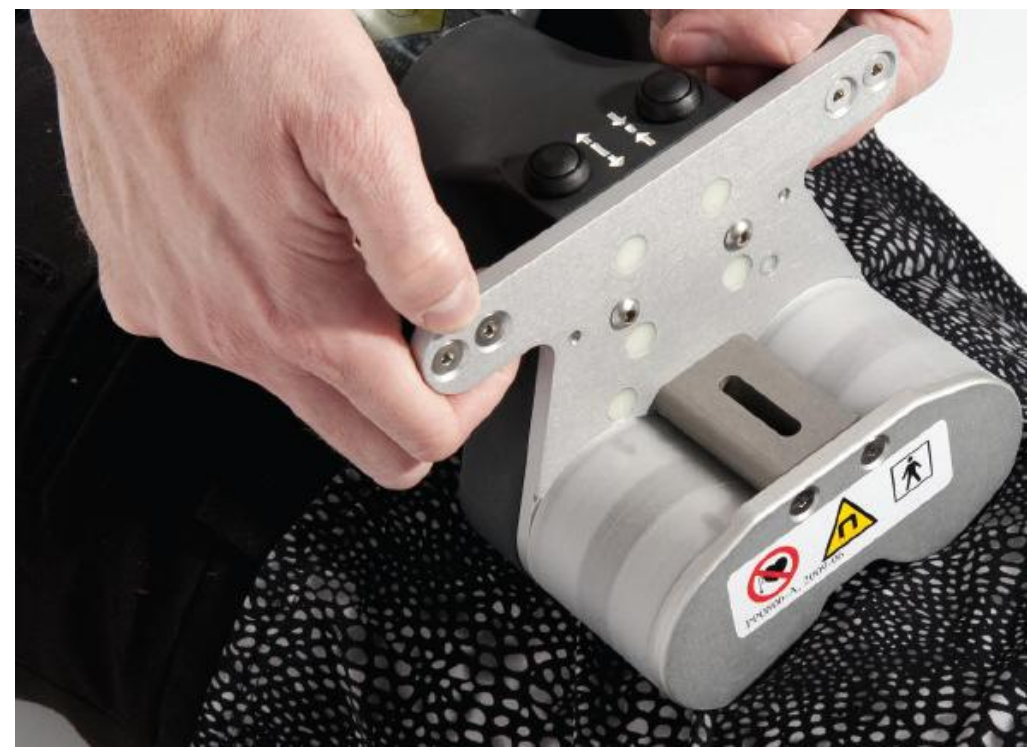

Abb.36:

Implantierter VEPTR mit integriertem Ellipse MAGEC TM Spinal System (rote Pfeile) bei Patient G. W..

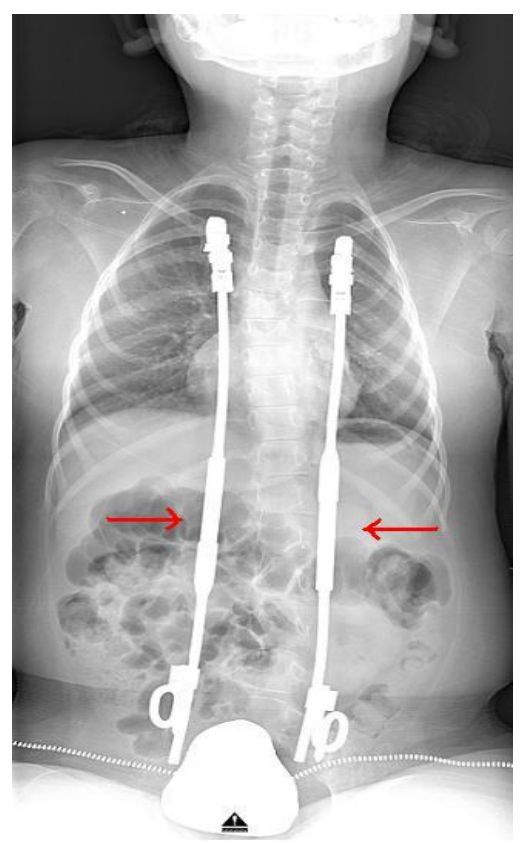




\section{Zusammenfassung}

Die bisherige Annahme war, dass sich bei Kindern, die mit dem VEPTR (vertical expandable prosthetic titanium rib)-Implantat versorgt wurden, aufgrund der geringeren Manipulation an der Wirbelsäule seltener und später Ossifikationen bzw. Fusionen entwickeln als beim growing-rod-Verfahren (Yazici und Emans 2009). Um diese Hypothesen zu prüfen, wurden in unserer Studie Kinder $(n=57)$ analysiert, die mittels VEPTR behandelt worden waren. Das durchschnittliche Alter der Kinder zur Zeit der primären VEPTR-Implantation lag bei 7,66 (1,33 bis 18,25) Jahren und die Nachuntersuchungszeit betrug im Mittel 29,8 (1 bis 101) Monate. Anhand von radiologischen Aufnahmen $(n=1328)$ wurden das Vorhandensein und die Ausdehnung von Ossifikationen erfasst und Daten zur Implantat-Wanderung gewonnen. Weitere Aspekte wie die Komplikationen Implantat-Infektion, -Ausriss und -Bruch als auch radiologische Parameter wie die Rippen-Spreizung nach PrimärImplantation und der Cobb- bzw. Kyphose-Winkel wurden untersucht. Die Messungen erfolgten bei den digitalisierten Röntgenaufnahmen mit dem Verarbeitungsprogramm Centricity ${ }^{\mathrm{TM}}$, bei den konventionellen Röntgenbildern mit dem Geometriedreieck und wurden statistisch mittels statistica 9.0 ausgewertet.

Es konnte gezeigt werden, dass bei $\mathbf{2 4 \%}$ der Patienten Ossifikationen auftraten, die in $92 \%$ der Fälle die Hauptimplantate der VEPTR-Systeme involvierten. Die Lendenwirbelsäule war zu 54\%, die Rippen und der Beckenkamm jeweils zu 23\% betroffen. Bei $\mathbf{1 1} \%$ der Kinder trat eine Ossifikation in den ersten zwölf Monaten und bei $19 \%$ in den ersten 24 Monaten auf. Die Rate an Ossifikationen nahm im Verlauf der Behandlung nicht zu. Nach 21 Monaten Behandlungsdauer waren 74\% der Patienten nicht ossifiziert und nach 53 Monaten blieben 52\% der Kinder ohne Verknöcherung. Die Größe und das Wachstum wiesen eine große Variabilität auf. Vermeintliche Einflussgrößen (Grunderkrankung, VEPTR-System, etc.) hatten im Mittel keinen signifikanten Einfluss auf das Auftreten einer Ossifikation. Bei Patienten mit einer Ossifikation zeigte sich durchschnittlich eine signifikant kleinere $\left(24,7^{\circ} \mathrm{zu}\right.$ $36,3^{\circ}$ ) Differenz der Cobb-Winkel prä und post Primär-Implantation. Ob ein Patient mit Ossifikation einen vermehrten Operationsaufwand bzw. mehr Komplikationen wie Implantat-Bruch, -Infektion und -Ausriss hatte, konnte in der vorliegenden Analyse aufgrund der geringen Fallzahl nicht abschließend geklärt werden. 
Eine Implantat-Wanderung fand asymptomatisch bei nahezu allen Patienten statt. Das Implantat „Rippe zu Rippe auf der konkaven Seite“ wanderte am zweithäufigsten (91\%), dafür aber die geringste Strecke. Am häufigsten (93\%) und am stärksten wanderte im Mittel das Implantat "Rippe zu LWS auf der konkaven Seite“ (Faktor 4,5 bzw. 8 im Vergleich zum Implantat „Rippe zu Rippe auf der konkaven Seite“), gefolgt vom Implantat „Rippe zu Becken auf der konvexen Seite“ (2,5 bzw. 6) und dem Implantat „Rippe zu Becken auf der konkaven Seite“ (2 bzw. 5). Die Hauptimplantate wanderten im jeweiligen VEPTR-System signifikant stärker als die akzessorischen Implantate. Wenn eine Implantat-Wanderung stattfand, so war immer das gesamte VEPTR-Konstrukt in Bewegung.

Bei $19 \%$ der Kinder kam es zu einem Implantat-Ausriss, wobei die Implantatverankerung im Beckenkamm entgegen der bisherigen Annahme im vorliegenden Kollektiv stabiler war als die Abstützung am Wirbelbogen der Wirbelsäule (Akbarnia und Emans 2010, Campbell et al. 2004 a).

Vermeintliche Einflussgrößen (Grunderkrankung, Alter bei der Primär-Implantation, etc.) auf die Implantat-Wanderung waren bis auf eine Ausnahme nicht signifikant. Das Implantat „Rippe zu Becken auf der konkaven Seite“ wanderte im Mittel um den Faktor zwei signifikant stärker bei den 50\% der Patienten, die den größeren Winkel prä Primär-Implantation aufwiesen.

Entgegen der bisherigen Annahme konnte bei 24\% der Patienten, die mit dem vertical expandable prosthetic titanium rib (VEPTR) versorgt worden waren, Ossifikationen nachgewiesen werden, die sogar $\mathrm{zu} 11 \%$ im ersten Behandlungsjahr auftraten. Im Vergleich zum growing rod entstanden jedoch weniger Ossifikationen (Cahill et al. 2010). Ferner konnte gezeigt werden, dass im Gegensatz zu den Wirbelfusionen beim growing rod die Ossifikationen beim VEPTR - wie bei Yazici und Emans - entlang des Implantats und vorwiegend an den Verankerungen entstanden (Yazici und Emans 2009). Bei nahezu allen Kindern bestand eine asymptomatische Implantat-Wanderung, die bei 19\% der Patienten zu einem Implantat-Ausriss führte. Anhand der vorliegenden Daten wären mögliche Ansätze zur Vermeidung der beschriebenen Probleme eine gleichmäßigere Druckverteilung auf das Skelett und eine kontinuierliche Verlängerung der VEPTRInstrumentarien z.B. durch extern gesteuerte Magnetmechanismen. 


\section{Literaturverzeichnis}

Aebi M (2005): The adult scoliosis. Eur Spine J 14(10), 925-948

Akbarnia BA (2007): Management themes in early onset scoliosis. J Bone Joint Surg Am 89(Suppl 1), 42-54

Akbarnia BA, Emans JB (2010): Complications of growth-sparing surgery in early onset scoliosis. Spine 35(25), 2193-2204

Akbarnia BA, Marks DS, Boachie-Adjei O, Thompson AG, Asher MA (2005): Dual growing rod technique for the treatment of progressive early-onset scoliosis: a multicenter study. Spine 30(Suppl 17), 46-57

Akbarnia BA, Breakwell LM, Marks DS, McCarthy RE, Thompson AG, Canale SK, Kostial PN, Tambe A, Asher MA (2008): Dual growing rod technique followed for three to eleven years until final fusion: the effect of frequency of lengthening. Spine 33(9), 984-990

Alici E, Pinar H (1992): The Alici spinal system in the surgical treatment of scoliosis. Acta Orthop Belg 58(Suppl 1), 122-128

Andrew T, Piggott H. (1985): Growth arrest for progressive scoliosis: combined anterior and posterior fusion of the convexity. J Bone Joint Surg Br $\underline{67(2)}$, 193-197

AWMF online Leitlinien der Deutschen Gesellschaft für Orthopädie und orthopädische Chirurgie (DGOOC) und des Berufsverbandes der Ärzte für Orthopädie (BVO): Idiopathische Skoliose im Wachstumsalter. http://www.awmf.org/uploads/tx_szleitlinien/033-025_S1_Idiopathische_Skoliose_im_ Wachstumsalter_10-2009_10-2012.pdf 2009

Bess S, Akbarnia BA, Thompson GH, Sponseller PD, Shah SA, El Sebaie H, Boachie-Adjei O, Karlin LI, Canale S, Poe-Kochert C et al. (2010): Complications of growing-rod treatment for early-onset scoliosis: analysis of one hundred and forty patients. J Bone Joint Surg Am 92(15), 2533-2543

Betz RR, Harms J, Clements DH, Lenke LG, Lowe TG, Shufflebarger HL, Jeszenszky D, Beele B (1999): Comparison of anterior and posterior instrumentation for correction of adolescent thoracic idiopathic scoliosis. Spine 24(3), 225-239 
Bonjour JP, Theintz G, Law F, Slosman D, Rizzoli R (1994): Peak bone mass. Osteoporos Int 4(Suppl 1), 7-13

Bremer R (2003): Management der Spina bifida. Orthopade 32(1), 85-97; quiz 97-98

Brenner D, Elliston C, Hall E, Berdon W (2001): Estimated risks of radiationinduced fatal cancer from pediatric CT. AJR Am J Roentgenol 176(2), 289-296

Brunner R, Gebhard F (2002): Neurogene Wirbelsäulendeformitäten. Teil 1: Konservative und operative Behandlung von Deformitäten an der Wirbelsäule. Orthopade $\underline{31(1)}, 51-57$

Cahill PJ, Marvil S, Cuddihy L, Schutt C, Idema J, Clements DH, Antonacci MD, Asghar J, Samdani AF, Betz RR (2010): Autofusion in the immature spine treated with growing rods. Spine 35(22), E1199-1203

Campbell RM Jr, Hell-Vocke AK (2003): Growth of the thoracic spine in congenital scoliosis after expansion thoracoplasty. J Bone Joint Surg Am 85(3), 409-420

Campbell RM Jr, Smith MD (2007): Thoracic insufficiency syndrome and exotic scoliosis. J Bone Joint Surg Am 89(Suppl 1), 108-122

Campbell RM Jr, Smith MD, Mayes TE, Mangos J, Willey-Courand DB, Kose N, Pinero R, Alder M, Duong HL, Surber J (2003): The characteristics of thoracic insufficiency syndrome associated with fused ribs and congenital scoliosis. $J$ Bone Joint Surg Am 85(3), 399-408

Campbell RM Jr, Smith MD, Hell-Vocke AK (2004 a): Expansion thoracoplasty: the surgical technique of opening-wedge thoracostomy. Surgical technique. J Bone Joint Surg Am 86(Suppl 1), 51-64

Campbell RM Jr, Smith MD, Mayes TC, Mangos JA, Willey-Courand DB, Kose N, Pinero RF, Alder ME, Duong HL, Surber JL (2004 b): The effect of opening wedge thoracostomy on thoracic insufficiency syndrome associated with fused ribs and congenital scoliosis. J Bone Joint Surg Am 86(8), 1659-1674

Carter OD, Haynes S (1987): Prevalence rates for scoliosis in US adults: Results from the first national health and nutrition examination survey. Int J Epidemiol 16(4), 537-544 
Connor JM, Conner AN, Connor RA, Tolmie JL, Yeung B, Goudie D (1987): Genetic aspects of early childhood scoliosis. Am J Med Genet 27(2), 419-424

Cotrel Y, Dubousset J, Guillaumat M (1988): New universal instrumentation in spinal surgery. Clin Orthop Relat Res $\underline{227}, 10-23$

Debnath UK (2010): Current concepts in the management of early-onset idiopathic scoliosis. Pediatr Health $\underline{4(3)}$, 343-354

De Wals P, Tairou F, Van Allen MI, Uh SH, Lowry RB, Sibbald B, Evans JA, Van den Hof MC, Zimmer P, Crowley M et al. (2007): Reduction in neural-tube defects after folic acid fortification in Canada. N Engl J Med 357(2), 135-142

Dickson RA, Stamper P, Sharp AM, Harker P (1980): School screening for scoliosis: cohort study of clinical course. Br Med J 281(6235), 265-267

Donnelly LF, Emery KH, Brody AS, Laor T, Gylys-Morin VM, Anton CG, Thomas SR, Frush DP (2001): Minimizing radiation dose for pediatric body applications of singledetector helical CT: strategies at a large Children's Hospital. AJR Am J Roentgenol 176(2), 303-306

Dubousset J, Cotrel Y (1991): Application technique of Cotrel-Dubousset instrumentation for scoliosis deformities. Clin Orthop Relat Res 264, 103-110

Dubousset J, Herring JA, Shufflebarger H (1989): The crankshaft phenomenon. J Pediatr Orthop 9(5), 541-550

Dwyer AF, Newton NC, Sherwood AA (1969): An anterior approach to scoliosis. A preliminary report. Clin Orthop Relat Res $\underline{62}, 192-202$

Ellipse Technologies, Inc.: Ellipse MAGEC ${ }^{\mathrm{TM}}$ Spinal System. Remote Control Technology for the Treatment of Scoliosis (Spine); Ellipse Technologies, Inc., Irvine 2010

Emans JB, Caubet JF, Ordonez CL, Lee EY, Ciarlo M (2005): The treatment of spine and chest wall deformities with fused ribs by expansion thoracostomy and insertion of vertical expandable prosthetic titanium rib: growth of thoracic spine and improvement of lung volumes. Spine 30(Suppl 17), 58-68 
Evans GA, Drennan JC, Russman BS (1981): Functional classification and orthopaedic management of spinal muscular atrophy. J Bone Joint Surg $\mathrm{Br} \underline{63(4)}$, 516-522

Facanha-Filho FA, Winter RB, Lonstein JE, Koop S, Novacheck T, L'Heureux EA Jr, Noren CA (2001): Measurement accuracy in congenital scoliosis. J Bone Joint Surg Am 83(1), 42-45

Farley FA, Loder RT, Nolan BT, Dillon MT, Frankenburg EP, Kaciroti NA, Miller JD, Goldstein SA, Hensinger RN (2001): Mouse model for thoracic congenital scoliosis. J Pediatr Orthop 21(4), 537-540

Ferreira JH, de Janeiro R, James Jl (1972): Progressive and resolving infantile idiopathic scoliosis. The differential diagnosis. J Bone Joint Surg Br 54(4), 648-655

Flynn JM, Ramirez N, Emans JB, Smith JT, Mulcahey MJ, Betz RR (2011): Is the vertebral expandable prosthetic titanium rib a surgical alternative in patients with spina bifida? Clin Orthop Relat Res 469(5), 1291-1296

Giehl JP, Zielke K, Hack HP (1989): Die Ventrale Derotationsspondylodese nach Zielke. Orthopade 18(2), 101-117

Goldberg CJ, Moore DP, Fogarty EE, Dowling FE (2008): Scoliosis: a review. Pediatr Surg Int 24(2), 129-144

Gollogly S, Smith JT, Campbell RM Jr (2004): Determining lung volume with threedimensional reconstructions of CT scan data: A pilot study to evaluate the effects of expansion thoracoplasty on children with severe spinal deformities. J Pediatr Orthop $\underline{24(3)}, 323-328$

Grivas TB, Vasiliadis E, Chatziargiropoulos T, Polyzois VD, Gatos K (2003): The effect of a modified Boston brace with anti-rotatory blades on the progression of curves in idiopathic scoliosis: aetiologic implications. Pediatr Rehabil 6(3-4), 237-242

Halm H, Richter A, Thomsen B, Köszegvary M, Ahrens M, Quante M (2009): Ventrale Skolioseoperationen. Stand der Technik und Vergleich mit dorsalen Verfahren. Orthopade 38(2), 131-134, 136-140, 142-145

Harrington PR (1962): Treatment of scoliosis. Correction and internal fixation by spine instrumentation. J Bone Joint Surg Am 44(4), 591-610 
Hasler CC, Mehrkens A, Hefti F (2010): Efficacy and safety of VEPTR instrumentation for progressive spine deformities in young children without rib fusions. Eur Spine J 19(3), 400-408

Hefti FL, McMaster MJ (1983): The effect of the adolescent growth spurt on early posterior spinal fusion in infantile and juvenile idiopathic scoliosis. J Bone Joint Surg $\mathrm{Br} \underline{65(3)}, 247-254$

Hell AK: Operationsmethode, Ergebnisse und Wachstumsanalyse von Kindern mit kongenitaler und neurogener Skoliose nach Expansionsthorakoplastik und vertical expandable prosthetic titanium rib (VEPTR) Implantation. Med. Habil.-Schr. Göttingen 2004

Hell AK, Göttingen: Mündliche Mitteilung 2011

Hell AK, Hefti F, Campbell RM Jr (2004): Behandlung der kongenitalen Skoliose mit dem vertical expandable prosthetic titanium rib (VEPTR) Implantat. Orthopade 33(8), 911-918

Hell AK, Campbell RM Jr, Hefti F (2005 a): Neues Therapiekonzept für Kinder mit Thoraxinsuffizienz-Syndrom aufgrund von Wirbelsäulenfehlbildungen. Klin Padiatr 217(5), 268-273

Hell AK, Campbell RM Jr, Hefti F (2005 b): The vertical expandable prosthetic titanium rib implant for the treatment of thoracic insufficiency syndrome associated with congenital and neuromuscular scoliosis in young children. J Pediatr Orthop B 14(4), 287-293

Hopf C (2000): Kriterien zur Behandlung idiopathischer Skoliosen zwischen 40 und 50․ Operative vs. konservative Therapie. Orthopade 29(6), 500-506

Hueter C (1862): Anatomische Studien an den Extremitätengelenken Neugeborener und Erwachsener. Virchows Arch Pathol Anat 25, 272

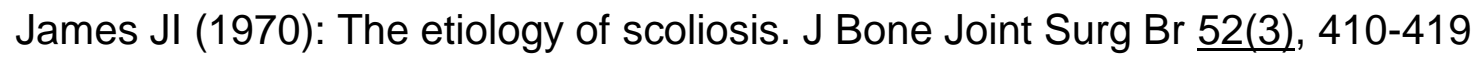

James JI (1971): Infantile idiopathic scoliosis. Clin Orthop Relat Res $\underline{77}, 57-72$

Jiang Y, Zhao Y, Wang YP, Qiu GX, Weng XS, Li Y (2011): Lung function after growing rod surgery for progressive early-onset scoliosis: a preliminary study. Chin Med J 124(23), 3858-3863 
Keiser RP, Shufflebarger HL (1976): The Milwaukee brace in idiopathic scoliosis: evaluation of 123 completed cases. Clin Orthop Relat Res $\underline{118}, 19-24$

Klemme WR, Denis F, Winter RB, Lonstein JW, Koop SE (1997): Spinal instrumentation without fusion for progressive scoliosis in young children. J Pediatr Orthop 17(6), 734-742

Klöckner C, Walter G, Matussek J, Weber U (2000): Ventrodorsal correction and instrumentation in idiopathic scoliosis. Orthopade 29(6), 571-577

Kotwicki T, Pietrzak S, Szulc A (2002): Three-dimensional action of Chêneau brace on thoracolumbar scoliosis. Stud Health Technol Inform 88, 226-229

Letellier K, Azeddine B, Blain S, Turgeon I, Wang DS, Boiro MS, Moldovan F, Labelle H, Poitras B, Rivard CH et al. (2007): Récents progrès dans l'étiopathogénie de la scoliose idiopathique de l'adolescent et nouveaux concepts moléculaires. médecine/sciences 23(11), 7

Letts RM, Bobechko WP (1974): Fusion of the scoliotic spine in young children. Effect of prognosis and growth. Clin Orthop Relat Res $\underline{101}$, 136-145

Liljenqvist U, Halm H (1998): Augmentation der VDS durch DoppelstabInstrumentation. Eine kritische Analyse der 2-bis 4-Jahres-Ergebnisse. Z Orthop Ihre Grenzgeb 136(1), 50-56

Loder R, Urquhart A, Steen H, Graziano G, Hensinger RN, Schlesinger A, Schork A, Shyr MA (1995): Variability in Cobb angle measurements in children with congenital scoliosis. J Bone Joint Surg Br $\underline{77(5)}$, 768-770

Lonstein JE: Congenital Deformities. In: Spine: State of the Art Reviews, Vol. 14(1); Hanley \& Belfus, Inc., Philadelphia 2000, 191-208

Lorber J (1966): Incidence and epidemiology of myelomeningocele. Clin Orthop Relat Res $\underline{45}, 81-83$

Lüder S, Schulte FJ (1989): Prävalenz und geographische Verteilung der Spina bifida aperta in der Bundesrepublik Deutschland. Klin Padiatr 201(2), 73-77

Luque ER (1982): Segmental spinal instrumentation for correction of scoliosis. Clin Orthop Relat Res $\underline{163}, 192-198$ 
Mannherz, RE, Betz RR, Clancy M, Steel HH (1988): Juvenile idiopathic scoliosis followed to skeletal maturity. Spine 13(10), 1087-1090

Marchesi D, Rüdeberg A, Aebi M (1991): Évolution des scolioses traitées conservativement chez les patients atteints de myéloméningocèle (patients des années 1964-1977). Acta Orthop Belg 57(4), 390-398

Marks DS, lqbal MJ, Thompson AG, Piggott H (1996): Convex spinal epiphysiodesis in the management of progressive infantile idiopathic scoliosis. Spine $\underline{21(16)}, 1884-$ 1888

Martinez MD, Schmid GJ, McKenzie JA, Ornitz DM, Silva MJ (2010): Healing of nondisplaced fractures produced by fatigue loading of the mouse ulna. Bone $\underline{46(6)}$, 1604-1612

McCarthy RE, Campbell RM, Hall JE. Infantile and juvenile idiopathic scoliosis. In: Spine: State of the Art Reviews, Vol. 14(1); Hanley \& Belfus, Inc., Philadelphia 2000, $163-180$

McMaster MJ (1998): Congenital scoliosis caused by unilateral failure of vertebral segmentation with contralateral hemivertebrae. Spine 23(9), 998-1005

McMaster MJ, Ohtsuka K (1982): The natural history of congenital scoliosis. A study of two hundred and fifty-one patients. J Bone Joint Surg Am $\underline{64(8)}, 1128-1147$

Moe JH, Kettleson DN (1970): Idiopathic scoliosis. Analysis of curve patterns and the preliminary results of Milwaukee-brace treatment in one hundred sixty-nine patients. J Bone Joint Surg Am 52(8), 1509-1533

Moe JH, Kharrat K, Winter RB, Cummine JL (1984): Harrington instrumentation without fusion plus external orthotic support for the treatment of difficult problems in young children. Clin Orthop Relat Res $\underline{185}$, 35-45

Montgomery F, Willner S (1997): The natural history of idiopathic scoliosis. Incidence of treatment in 15 cohorts of children born between 1963 and 1977. Spine 22(7), $772-774$

Mostacciuolo ML, Danieli GA, Trevisan C, Müller E, Angelini C (1992): Epidemiology of spinal muscular atrophies in a sample of the Italian population. Neuroepidemiology $\underline{11(1)}, 34-38$ 
Motoyama EK, Yang CI, Deeney VF (2009): Thoracic malformation with early onset scoliosis: effect of serial VEPTR expansion thoracoplasty on lung growth and function in children. Peadiatr Respir Rev 10(1), 12-17

Müller EB, Nordwall A (1992): Prevalence of scoliosis in children with myelomeningocele in western Sweden. Spine 17(9), 1097-1102

Murray JF: The normal lung: the basis for diagnosis and treatment of pulmonary disease., 2nd edition; W. B. Saunders Company, Philadelphia 1986, 46

Nachemson AL, Peterson LE (1995): Effectiveness of treatment with a brace in girls who have adolescent idiopathic scoliosis. A prospective, controlled study based on data from the Brace Study of the Scoliosis Research Society. J Bone Joint Surg Am $\underline{77(6)}, 815-822$

Paterson A, Frush DP, Donnelly LF (2001): Helical CT of the body: are settings adjusted for pediatric patients? AJR Am J Roentgenol 176(2), 297-301

Pearn J (1980): Classification of spinal muscular atrophies. Lancet $\underline{315(8174)}$, 919922

Pfandlsteiner T, Wallnoefer P, Wimmer C (2010): Wachstumslenkende operative Behandlung der juvenilen Skoliose mit USS paediatric. Oper Orthop Traumatol 22(2), 149-163

Piggott $\mathrm{H}$ (1980): The natural history of scoliosis in myelodysplasia. J Bone Joint Surg $\operatorname{Br} \underline{62(1)}, 54-58$

Ramirez N, Flynn JM, Serrano JA, Carlo S, Cornier AS (2009): The Vertical Expandable Prosthetic Titanium Rib in the treatment of spinal deformity due to progressive early onset scoliosis. J Pediatr Orthop B 18(4), 197-203

Riddick MF, Winter RB, Lutter LD (1982): Spinal deformities in patients with spinal muscle atrophy: a review of 36 patients. Spine $\underline{7(5)}, 476-483$

Rigo M, Reiter C, Weiß HR (2003): Effect of conservative management on the prevalence of surgery in patients with adolescent idiopathic scoliosis. Pediatr Rehabil $\underline{6(3-4)}, 209-214$

Rinsky LA, Gamble JG, Bleck EE (1985): Segmental instrumentation without fusion in progressive scoliosis. J Pediatr Orthop $\underline{5(6)}, 687-690$ 
Riseborough EJ, Wynne-Davies R (1973): A genetic survey of idiopathic scoliosis in Boston, Massachusetts. J Bone Joint Surg Am 55(5), 974-982

Robinson CM, McMaster MJ (1996): Juvenile idiopathic scoliosis. Curve patterns and prognosis in one hundred and nine patients. J Bone Joint Surg Am 78(8), 1140-1148

Samdani AF, Ranade A, Dolch HJ, Williams R, St Hilaire T, Cahill P, Betz RR (2009) Bilateral use of the vertical expandable prosthetic titanium rib attached to the pelvis: a novel treatment for scoliosis in the growing spine. J Neurosurg Spine 10(4), 287292

Samuelsson L, Eklöf O (1988): Scoliosis in myelomeningocele. Acta Orthop Scand 59(2), 122-127

Sankar WN, Acevedo DC, Skaggs DL (2010): Comparison of complications among growing spinal implants. Spine $\underline{35(23)}$, 2091-2096

Schmorl G, Junghanns H: Die gesunde und die kranke Wirbelsäule in Röntgenbild und Klinik., 5. Auflage; Georg Thieme Verlag, Stuttgart 1968

Schwab F, Dubey A, Gamez L, El Fegoun AB, Hwang K, Pagala M, Farcy JP (2005): Adult scoliosis: prevalence, SF-36, and nutritional parameters in an elderly volunteer population. Spine $\underline{30(9)}, 1082-1085$

Schwentker EP, Gibson DA (1976): The orthopaedic aspects of spinal muscular atrophy. J Bone Joint Surg Am 58(1), 32-38

Shufflebarger HL, Clark CE (1991): Prevention of the crankshaft phenomenon. Spine 16(8), 409-411

Smith JT (2011): Bilateral rib-to-pelvis technique for managing early-onset scoliosis. Clin Orthop Relat Res 469(5), 1349-1355

Stokes IA (1994): Three-dimensional terminology of spinal deformity. A report presented to the Scoliosis Research Society by the Scoliosis Research Society Working Group on 3-D terminology of spinal deformity. Spine 19(Suppl 2), 236-248

Sucato DJ (2007): Spine deformity in spinal muscular atrophy. J Bone Joint Surg Am 89(Suppl 1), 148-154

Synthes $\AA^{\circledR}$ : VEPTR, vertical expandable prosthetic titanium rib. A new treatment 
concept for paediatric three-dimensional thoracic deformities.; Synthes ${ }^{\circledR}$, Stratec Medical, Oberdorf 2004

Tello CA (1994): Harrington instrumentation without arthrodesis and consecutive distraction program for young children with severe spinal deformities. Experience and technical details. Orthop Clin North Am 25(2), 333-351

Thompson GH, Akbarnia BA, Kostial P, Poe-Kochert C, Armstrong DG, Roh J, Lowe R, Asher MA, Marks DS (2005): Comparison of single and dual growing rod techniques followed through definitive surgery: a preliminary study. Spine $\underline{30(18)}$, 2039-2044

Thompson GH, Lenke LG, Akbarnia BA, McCarthy RE, Campbell RM Jr (2007): Early onset scoliosis: future directions. J Bone Joint Surg Am 89(Suppl 1), 163-166

Tolo VT, Gillespie R (1978): The characteristics of juvenile idiopathic scoliosis and results of its treatment. J Bone Joint Surg $\mathrm{Br} \underline{60(2)}, 181-188$

Trivedi J, Thomson JD, Slakey JB, Banta JV, Jones PW (2002): Clinical and radiographic predictors of scoliosis in patients with myelomeningocele. $J$ Bone Joint Surg Am 84(8), 1389-1394

Tsou PM, Yau A, Hodgson AR (1980): Embryogenesis and prenatal development of congenital vertebral anomalies and their classification. Clin Orthop Relat Res $\underline{152}$, 211-232

Uthgenannt BA, Kramer MH, Hwu JA, Wopenka B, Silva MJ (2007): Skeletal selfrepair: stress fracture healing by rapid formation and densification of woven bone. $J$ Bone Miner Res 22(10), 1548-1556

Volkmann R: Die Krankheiten der Bewegungsorgane. In: Handbuch der allgemeinen und speziellen Chirurgie; hrsg. v. Billroth T, von Pitha F; Enke, Stuttgart 1869, 694696

Waldhausen JH, Redding GJ, Song KM (2007): Vertical expandable prosthetic titanium rib for thoracic insufficiency syndrome: a new method to treat an old problem. J Pediatr Surg 42(1), 76-80

Weinstein SL (1999): Natural history. Spine 24(24), 2592-2600

Weiß HR, Weiß G, Petermann F (2003): Incidence of curvature progression in 
idiopathic scoliosis patients treated with scoliosis in-patient rehabilitation (SIR): an age- and sex-matched cotrolled study. Pediatr Rehabil $\underline{6}, 23-30$

Weiß HR, Negrini S, Rigo M, Kotwicki T, Hawes MC, Grivas TB, Maruyama T, Landauer $F$ (2006): Indications for conservative management of scoliosis (guidelines). Scoliosis 1,5

Willner S, Udén A (1982): A prospective prevalence study of scoliosis in southern Sweden. Acta Orthop Scand $\underline{53}, 233-237$

Wimmer C, Wallnoefer P, Pfandlsteiner T (2010): Die operative Behandlung von Skoliosen mit dem „vertical expandable prosthetic titanium rib“ (VEPTR). Oper Orthop Traumatol 22(2), 123-136

Winter RB (1973): Congenital scoliosis. Clin Orthop Relat Res $\underline{93}$, 75-94

Winter RB (1981): Convex anterior and posterior hemi-arthrodesis and epiphysiodesis in young children with progressive congenital scoliosis. J Pediatr Orthop 1(4), 361-366

Winter RB, Moe JH (1982): The results of spinal arthrodesis for congenital spine deformity in patients younger than 5 years old. J Bone Joint Surg Am 64(3), 419-432 Winter RB, Moe JH, Eilers VE. (1968): Congenital Scoliosis: A study of 234 patients treated and untreated. J Bone Joint Surg Am 50(1), 1-47

Wynne-Davies R (1968): Familial (idiopathic) scoliosis. A family survey. J Bone Joint Surg $\operatorname{Br} \underline{50(1)}, 24-30$

Yazici M, Emans J (2009): Fusionless instrumentation systems for congenital scoliosis: expandable spinal rods and vertical expandable prosthetic titanium rib in the management of congenital spine deformities in the growing child. Spine $\underline{34(17)}$, 1800-1807

Zielke K (1982): Ventrale Derotationsspondylodese. Behandlungsergebnisse bei idiopathischen Lumbalskoliosen. Z Orthop Ihre Grenzgeb 120(3), 320-329 


\section{Danksagung}

Ich möchte mich herzlich bei meiner Doktormutter Prof. Dr. med. A.-K. Hell bedanken, die mich sehr gut betreute und mir bei Fragen stets hilfreich zur Seite stand.

Des Weiteren möchte ich mich beim Team der Abteilung für Medizinische Statistik der Universitätsmedizin Göttingen (insbesondere bei Dr. rer. nat. Klaus Jung und David Ellenberger) für die Unterstützung bei der statistischen Auswertung bedanken.

Ferner möchte ich mich bei Tanja Freitag für die Hilfe bei der Benutzung des Textprogramms Word bedanken.

Mein liebster Dank geht an meine Freundin Katharina Horst für die wiederholte Begutachtung der Arbeit auf grammatikalische Fehler sowie die Verbesserung des Ausdrucks, aber vor allem für die viele Zeit, in der sie mir mit Rat und Tat zur Seite stand. 